FELIPE NEVES SOUZA

CARACTERIZAÇÃO ELÉTRICA DE TÚNEL-FET EM ESTRUTURA DE NANOFIO COM FONTES DE SiGe E Ge EM FUNÇÃO DA TEMPERATURA 
FELIPE NEVES SOUZA

\section{CARACTERIZAÇÃO ELÉTRICA DE TÚNEL-FET EM ESTRUTURA DE NANOFIO COM FONTES DE SiGe E Ge EM FUNÇÃO DA TEMPERATURA}

\footnotetext{
Tese apresentada à Escola Politécnica da Universidade de São Paulo para a obtenção do título de Doutor em Ciências.
} 
FELIPE NEVES SOUZA

\section{CARACTERIZAÇÃO ELÉTRICA DE TÚNEL-FET EM ESTRUTURAS DE NANOFIO COM FONTES DE SiGe E Ge EM FUNÇÃO DA TEMPERATURA}

Tese apresentada à Escola Politécnica da Universidade de São Paulo para a obtenção do título de Doutor em Ciências.

Área de Concentração:

Engenharia Elétrica / Microeletrônica.

Orientador: Prof. Dr. João Antonio Martino Coorientadora: Prof. ${ }^{a}$ Dr. ${ }^{\text {a }}$ Paula G. der Agopian 
Dedico este trabalho aos meus pais, Milton e Elisabete, e a minha família, os quais amo muito e devo tudo que sou. 


\section{AGRADECIMENTOS}

Ao professor. Dr. João Antonio Martino, não somente pela dedicação e orientação, mas pela oportunidade que me foi dada, pelo voto de confiança e toda compreensão nos momentos de dificuldade ao longo desses quatro anos.

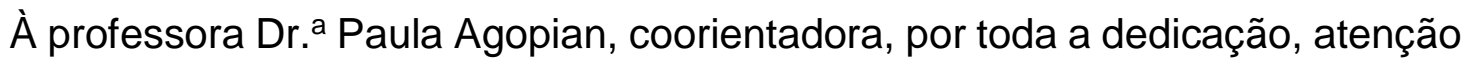
e apoio que sempre depositou em mim, contribuindo para a realização desse doutorado.

Aos professores e pesquisadores Cor Claeys, Eddy Simoen, Rita Rooyackers, e Anne Vandooren por me receberem no centro de pesquisa Imec, Bélgica, colaborando para o enriquecimento desta tese.

Aos amigos do grupo SOI-CMOS do LSI/EPUSP pela ajuda e discussões ao longo deste trabalho, pela convivência e momentos de descontração.

Aos meus amigos da KULeuven e Imec pelo companheirismo e apoio durante minha estadia na Bélgica.

À CAPES, pelo suporte financeiro indispensável para a realização deste trabalho.

Aos meus pais, Milton e Elisabete, às minhas irmãs, Thaís e Amanda, e aos meus familiares, pelo constante incentivo e apoio em todas as minhas escolhas, e por acreditarem em todos os meus projetos.

À minha querida amiga e namorada Pâmella, por estar sempre ao meu lado, com seu apoio e dedicação, que contribuíram para que este trabalho fosse possível. Agradeço pela compreensão nos muitos momentos de ausência.

Aos demais amigos e tantos outros que de alguma forma colaboraram para a realização deste trabalho e que, de forma involuntária, foram omitidos. 
É o trabalho que nunca se começa que leva mais tempo a terminar.

J. R. R. Tolkien 


\section{RESUMO}

Este trabalho teve como objetivo estudar os transistores de tunelamento por efeito de campo em estruturas de nanofio (NW-TFET), sendo realizado através de analises com base em explicações teóricas, simulações numéricas e medidas experimentais.

A fim de avaliar melhorar o desempenho do NW-TFET, este trabalho utilizou dispositivos com diferentes materiais de fonte, sendo eles: Si, liga SiGe e Ge, além da variação da espessura de $\mathrm{HfO}_{2}$ no material do dielétrico de porta.

Com o auxílio de simulações numéricas foram obtidos os diagramas de bandas de energia dos dispositivos NW-TFET com fonte de $\mathrm{Si}_{0,73} \mathrm{Ge}_{0,27}$ e foi analisada a influência de cada um dos mecanismos de transporte de portadores para diversas condições de polarização, sendo observado a predominância da influência da recombinação e geração Shockley-Read-Hall (SRH) na corrente de desligamento, do tunelamento induzido por armadilhas (TAT) para baixos valores de tensões de porta $\left(0,5 \mathrm{~V}>\mathrm{V}_{\mathrm{GS}}>1,5 \mathrm{~V}\right)$ e do tunelamento direto de banda para banda (BTBT) para maiores valores tensões de porta $\left(\mathrm{V}_{\mathrm{GS}}>1,5 \mathrm{~V}\right)$. A predominância de cada um desses mecanismos de transporte foi posteriormente comprovada com a utilização do método de Arrhenius, sendo este método adotado em todas as análises do trabalho.

O comportamento relativamente constante da corrente dos NW-TFETs com a temperatura na região de BTBT tem chamado a atenção e por isso foi realizado o estudo dos parâmetros analógicos em função da temperatura. Este estudo foi realizado comparando a influência dos diferentes materiais de fonte. O uso de Ge na fonte, permitiu a melhora na corrente de tunelamento, devido à sua menor banda proibida, aumentando a corrente de funcionamento (IoN) e a transcondutância do dispositivo. Porém, devido à forte dependência de BTBT com o campo elétrico, o uso de Ge na fonte resulta em uma maior degradação da condutância de saída. Entretanto, a redução da espessura de $\mathrm{HfO}_{2}$ no dielétrico de porta resultou no melhor acoplamento eletrostático, também aumentando a corrente de tunelamento, fazendo com que o dispositivo com fonte $\mathrm{Ge}$ e menor $\mathrm{HfO}_{2}$ apresentasse melhores resultados analógicos quando comparado ao puramente de Si. 
O uso de diferentes materiais durante o processo de fabricação induz ao aumento de defeitos nas interfaces do dispositivo. Ao longo deste trabalho foi realizado o estudo da influência da densidade de armadilhas de interface na corrente do dispositivo, demonstrando uma relação direta com o TAT e a formação de uma região de platô nas curvas de IDS $\times V_{G S}$, além de uma forte dependência com a temperatura, aumentando a degradação da corrente para temperaturas mais altas.

Além disso, o uso de Ge introduziu maior número de impurezas no óxido, e através do estudo de ruído foi observado que o aumento na densidade de armadilhas no óxido resultou no aumento do ruído flicker em baixa frequência, que para o TFET, ocorre devido ao armadilhamento e desarmadilhamento de elétrons na região do óxido. E mais uma vez, o melhor acoplamento eletrostático devido a redução da espessura de $\mathrm{HfO}_{2}$, resultou na redução desse ruído tornando-se melhor quando comparado à um TFET puramente de Si.

Neste trabalho foi proposto um modelo de ruído em baixa frequência para o NW-TFET baseado no modelo para MOSFET. Foram realizadas apenas algumas modificações, e assim, obtendo uma boa concordância com os resultados experimentais na região onde o BTBT é o mecanismo de condução predominante.

Palavras chaves: TFET. Nanofio. Tunelamento de banda para banda. Tunelamento induzido por armadilha. Influência da temperatura. Parâmetros analógicos. Ruído flicker. Histerese. 


\begin{abstract}
This work aims to study the nanowire tunneling field effect transistors (NWTFET). The analyses were performed based on theoretical explanations, numerical simulations and experimental data.

In order to improve the NW-TFET performance, it was used devices with different source compositions, such as $\mathrm{Si}$, SiGe alloy and Ge, besides different thicknesses of $\mathrm{HfO}_{2}$ for the gate dielectric.

With the aid of numerical simulations it was obtained the NW-TFET energy band diagrams and analyzed the influence of recombination and generation Shockley-ReadHall (SRH) on the off current, the influence of the trap assisted tunneling (TAT) at low gate voltage bias $\left(0,5 \mathrm{~V}>\mathrm{V}_{\mathrm{GS}}>1,5 \mathrm{~V}\right)$ and the direct band to band tunneling (BTBT) at higher gate voltage $\operatorname{bias}\left(\mathrm{V}_{G S}>1,5 \mathrm{~V}\right)$. The predominance of each conduction mechanisms was confirmed by the Arrhenius plot method, being this method adopted in all analysis in this work.
\end{abstract}

The constant current with the temperature in the BTBT region has drawn attention and due to that, this work have studied the NW-TFET analog performance as function of temperature and also the influence of the source composition. The Ge source device shows an improved tunneling current, related to the bandgap narrowing, which leads to higher lon and transconductance. However, due to the strong BTBT dependence with the electric field, the use of Ge as source results in further lon/loFf degradation. Despite this, the reduced $\mathrm{HfO}_{2}$ thickness in the gate dielectric, results in better electrostatic coupling, which also increases the tunneling current, making this device to present better analog performance when compared to devices with Si source.

The use of different materials during the device fabrication leads to an increase of the interface defects. This work presented the influence of the interface trap density on the current, showing a direct relation with TAT and appearance of a plateau region in the IDS $x V_{G S}$ curves. In addition it was shown a strong temperature dependence increasing the current degradation at higher temperatures. Furthermore, the use of $\mathrm{Ge}$ has shown an increase of impurities in the oxide, and through the noise study it was observed the flicker noise increase at low frequency, which for TFETs, occurs due to the electrons trapping and detrapping in the oxide region. Once again, the reduced 
$\mathrm{HfO}_{2}$ thickness leads to better electrostatic coupling, resulting in noise reduction and becoming better when compared to a devices with Si source.

In this work was proposed a low frequency noise model for a NW-TFET based on MOSFET models. Minor changes have been done, and thus a good agreement with the experimental results in the region where the BTBT is predominant conduction mechanism was obtained.

Keywords: TFET. Nanowire. Band to band tunneling. Trap assisted tunneling. Temperature influence. Analog parameters. Flicker noise. Hysteresis. 


\section{LISTA DE FIGURAS}

Figura 1.1 - Evolução tecnologia ao longo do tempo (7) ………….....................24

Figura 2.1 - Seção transversal de um TFET de porta de simples.

Figura 2.2 - Seção transversal de um TFET de múltiplas portas em estrutura horizontal (a) e vertical (b)

Figura 2.3 - Diagrama de bandas de energia para um nTFET polarizado com tensão de porta nula (a) e tensão de porta positiva (b) .29

Figura 2.4 - Ilha de potencial com barreira de potencial de $2 \mathrm{~nm}$ de largura e $40 \mathrm{mV}$ de altura (a). Função de onda (b) (32) .30

Figura 2.5 - Curva da $E_{g}$ do Si x T, calculado através da equação 2.12. .35

Figura 2.6 - Esquemático do SID em função de $f$ para um transistor MOSFET .38

Figura 3.1 - Etapas do processo de fabricação de um vertical NW-TFET com fonte de $\mathrm{Si}_{1-\mathrm{x}} \mathrm{Ge}_{\mathrm{x}}(22)$

Figura 3.2 - Esquemático da seção transversal (a) e o corte transversal (b) de um de um NW-TFET vertical.....

Figura 3.3 - Concentração de dopantes de um NW-TFET (50) ............................42

Figura 3.4 - Seção transversal de um NW-TFET indicando suas dimensões 43

Figura 3.5 - Curva de IDS $\times$ VGS para um NW-TFET com fonte de $\mathrm{Si}_{0,73} \mathrm{Ge}_{0,27}$ simulada no TCAD Sentaurus.

Figura 3.6 - Diagramas de bandas de energia para um NW-TFET com fonte de Sio,73 $\mathrm{Ge}_{0,27} \mathrm{em}$ diferentes condições de polarização, obtido por simulações no TCAD Sentaurus .46

Figura 3.7 - Curvas experimentais de IDS $x$ VGS para dispositivos com fonte de $\mathrm{Si}_{0,73} \mathrm{Ge}_{0,27}$ e diferentes diâmetros em função da temperatura 47

Figura 3.8 - Curva experimental de IDS $x V_{\text {GS }}$ para um TFET com fonte de $\mathrm{Si}_{0,73} \mathrm{Ge}_{0,27}$, apresentando a influência predominante dos mecanismos de condução em cada região de polarização .48

Figura 3.9 - Curva de $\mathrm{E}_{\mathrm{A}} \times \mathrm{V}_{\mathrm{GS}}$ para um NW-TFET com fonte de $\mathrm{Si}_{0,73} \mathrm{Ge}_{0,27}$. 49

Figura 3.10 - Relação lon/loff X T para NW-TFET com fonte de $\mathrm{Si}_{0,73} \mathrm{Ge}_{0,27}$ e dois diâmetros diferentes

Figura 3.11 - Curvas experimentais de IDS $\times$ VDS com diferentes valores de $V_{G S}$ e em função da temperatura para NW-TEFET com fonte de Sio,73Ge, $27 \ldots . . .51$ 
Figura 3.12 - Curvas experimentais de lDS $\times$ VDS para NW-TFET com fonte de $\mathrm{Si}_{0,73} \mathrm{Ge}_{0,27}$, com diâmetros diferentes e em função da temperatura ...52

Figura 3.13 - Curvas experimentais de IDS $x V_{\text {GS }}$ para NW-TFET com diferentes composições de fonte

Figura 3.14 - Curvas experimentais de IDS $x$ VDS para NW-TFET com diferentes composições de fonte

Figura 3.15 - Curvas experimentais de IDS $x V_{G S}$ em função da Temperatura para NW-TFET com fonte de $\mathrm{Si}_{1-x} \mathrm{Ge}$.

Figura 3.16 - Curvas experimentais de IDS $x$ VGS em função da Temperatura para NW-TFET com fonte de Si

Figura 3.17 - Curvas experimentais de $E_{A} \times V_{G S}$ para NW-TFET com diferentes composições de fonte .56

Figura 3.18 - Curvas experimentais de $g_{m} \times$ T para NW-TFET com diferentes composições de fonte .58

Figura 3.19 - Curvas experimentais de gd X T para NW-TFET com diferentes composições de fonte .59

Figura 3.20 - Curvas experimentais de Av x T para NW-TFET com diferentes composições de fonte.

Figura 3.21 - Curvas experimentais de IDS $x$ VGS, comparando NW-TFET com fontes de diferentes materiais

Figura 3.22 - Figura esquemática do dispositivo NW-TFET simulado, demonstrando onde foram considerados o $\mathrm{Nit}_{\text {it }}$

Figura 3.23 - Curvas IDS X VGS variando a quantidade de $\mathrm{N}_{\mathrm{iT}}$ na interface fonte/canal obtida por simulações no TCAD Silvaco

Figura 3.24 - Curvas comparando a influência de cada mecanismo de transporte de portadores no comportamento de IDS com a variação de $V_{G S}$ .63

Figura 3.25 - Curvas de IDS $\times V_{G S}$ variando o $E_{g}$ do material de fonte e considerando a influência de $\mathrm{N}_{\mathrm{it}}$

Figura 3.26 - Curvas experimentais de IDS $x V_{G S}$ em função da temperatura para NW-TFET com diferentes materiais de fonte 65

Figura 3.27 - Curvas simuladas de IDS $x V_{G S}$ comparando a influência do $N_{i t}$ na interface fonte/canal em função da temperatura

Figura 4.1 - Etapas do processo de fabricação de um vertical NW-TFET com fonte de $\mathrm{Ge}(23)$ 
Figura 4.2 - Corte transversal (a) e o Esquemático da seção transversal (b) de um de um NW-TFET

Figura 4.3 - Curvas experimentais de IDS $\times$ VGS para NW-TFETs com diferentes composições de fonte e espessura de $\mathrm{HfO}_{2}$ no dielétrico de porta 72

Figura 4.4 - Curva de IDS $x$ VDs para NW-TFETs com diferentes composições de fonte e espessura de $\mathrm{HfO}_{2}$ no dielétrico de porta 72

Figura 4.5 - Curva experimental $g_{m} \times V_{G S}$ para NW-TFETs com diferentes composições de fonte e espessura de $\mathrm{HfO}_{2}$ no dielétrico de porta .......73

Figura 4.6 - Curvas experimentais de $g_{d} \times V_{G s}$ para NW-TFETs com diferentes composições de fonte e espessura de $\mathrm{HfO} 2$ no dielétrico de porta .....74

Figura 4.7 - Curvas experimentais de $A v \times V_{G S}$ para NW-TFETs com diferentes composições de fonte e espessura de $\mathrm{HfO}_{2}$ no dielétrico de porta .......75

Figura 4.8 - Curvas experimentais de lDs $\times V_{\text {GS }}$ para os diferentes NW-TFETs estudados, sendo dois com fonte de Ge e diferentes espessuras de $\mathrm{HfO}_{2}$ e um com fonte de $\mathrm{Si}$. .77

Figura 4.9 - Curvas de $E_{A} \times V_{G s}$ para os diferentes NW-TFETs estudados .77

Figura 4.10 - Curvas experimentais de $S_{I D} \times f$ e diferentes polarizações de $V_{D S}$ para um NW-TFET com fonte de Ge. .79

Figura 4.11 - Curvas de SID x f para os três diferentes NW-TFETs estudados .80

Figura 4.12 - Curvas de $S_{I D} \times$ IDS extraídas em $f=25 \mathrm{~Hz}$ e $V_{D S}=0,9 \mathrm{~V}$ para os diferentes NW-TFETs estudados

Figura 4.13 - Curvas de SID normalizados por $\operatorname{IDS}^{2} \mathrm{x}$ inclinação de sublimiar (SS) para NW-TFETs com fonte de Ge.

Figura 4.14 - Curvas experimentais de SID normalizada por $\operatorname{lDS}^{2}$ e de $\left(g_{m} / I_{D S}\right)^{2} x \operatorname{lDS}$ para os diferentes NW-TFETs estudados .83

Figura 4.15 - Curvas de $g_{m} \times V_{G S}$ para os diferentes NW-TFETs .83

Figura 4.16 - Curvas de SVG $\times V_{G S}$ para $f=25 \mathrm{~Hz}$ e VDS $=0,9 \mathrm{~V}$ para três diferentes dispositivos estudados

Figura 4.17 -Curvas de IDS $\times V_{G S}$ obtidas de forma experimental e simuladas para um NW-TFET com fonte de Ge.

Figura 4.18 - Perfil de geração e recombinação devido à BTBT obtido por simulação ao longo do dispositivo .86

Figura 4.19 - Curva de SID x f para um transistor NW-MOSFET .87

Figura 4.20 - Curva experimental de SID x IDS para um transistor NW-MOSFET 
Figura 4.21 Curvas de SID x IDS para um NW-TFET com fonte de Ge obtidas de experimentalmente e o modelo proposto

Figura 4.22 Curva experimental de IDS $x$ VGs para um NW-TFET com T $=300 \mathrm{~K}$ e $77 \mathrm{~K}$

Figura 4.23 - Curvas de SID e SID normalizado por IDS $^{2}$ para um NW-TFET com fonte de $\mathrm{Ge}$ .90

Figura 4.24 - Curvas experimentais de SvG x IDs para um NW-TFET com fonte de Ge .90

Figura 4.25 - Curvas experimentais de lDs $\times V_{G S}$ em função da temperatura para transistores NW-TFET com espessuras de $\mathrm{HfO}_{2}$ de $3 \mathrm{~nm}(\mathrm{~A})$ e $2 \mathrm{~nm}$ (B)

Figura 4.26 - Curvas experimentais com dupla varredura de IDS $x V_{G S}$ em função da temperatura para transistores NW-TFET com espessuras de $\mathrm{HfO}_{2}$ de $3 \mathrm{~nm}(\mathrm{~A})$ e $2 \mathrm{~nm}(\mathrm{~B})$. .92

Figura 4.27 - Curva de $\Delta V_{\text {GS }} \times \mathrm{T}$ para o NW-TFET com $3 \mathrm{~nm}$ de $\mathrm{HfO}_{2} \ldots \ldots \ldots \ldots \ldots \ldots . . . . . .93$

Figura 4.28 - Curva de IDs $\times 1 /$ T para o NW-TFET com $\mathrm{HfO}_{2}$ de $3 \mathrm{~nm}$. .94

Figura 5.1: Curvas experimentais de IDS $\times V_{G S}(A)$ e IDS $\times V_{D S}(B)$ para transistores NW-TFET com diferentes composições de fonte .96

Figura 5.2: Curvas experimentais de $g_{m}(A)$ e $g_{d}(B)$ em função da porcentagem de

Ge na fonte .97

Figura 5.3: Curvas experimentais de Avem função da porcentagem de Ge na fonte 


\section{LISTA DE TABELAS}

Tabela 3-1 - Dimensões dos dispositivos NW-TFET fabricados no Imec, Bélgica e utilizados neste trabalho.

Tabela 4-1 - Dimensões dos dispositivos NW-TFET com fonte de Ge e Si fabricados no Imec, Bélgica e utilizados neste trabalho. .70

Tabela 4-2 - Diferença entre os dispositivos NW-TFET utilizados neste capítulo. ...70

Tabela 4-3 - Valores de $\Delta V_{\text {GS }}$ e Not em diferentes temperaturas para o NW-TFET com $3 \mathrm{~nm}$ de $\mathrm{HfO}_{2}$ .93

Tabela 5-1: Dimensões dos NW-TFET provenientes de diferentes processos de fabricação .95

Tabela A-1 - Modelos utilizados no TCAD Sentaurus e TCAD Silvaco 114 


\section{LISTA DE SIGLAS E ABREVIATURAS}

Cl Circuitos Integrados

c-Ge Germânio cristalino

BTBT Band to Band Tunneling (tunelamento de banda para banda)

EOT Equivalent Oxide Thickness (espessura equivalente de óxido)

g-r Geração e recombinação

$\mathrm{HCl} \quad$ Ácido clorídrico

$\mathrm{HfO}_{2} \quad$ Óxido de Háfnio

Imec Interuniversity Microelectronics Centre (Centro Interuniversitário de Microeletrônica)

i-Si Silício com concentração intrínseca de portadores.

KLA Klassen

MOS Metal-Oxide-Semiconductor (Metal-Óxido-Semicondutor)

MOSFET Metal-Oxide-Semiconductor Field-Effect Transistor (transistor de efeito de campo metal-óxido-semicondutor)

nTFET TFET tipo $\mathrm{n}$

NW Nanowire (nanofio)

NW-TFET Nanowire TFET (TFET em estrutura de nanofio)

PIN $\quad$ Estrutura com dopagens do tipo $\mathrm{p}$ - intrínseco - $\mathrm{n}$

PSD Power Specrtral Density (densidade espectral de potência)

PTFET TFET tipo $\mathrm{p}$

RTCVD Rapid Thermal Chemical Vapor Deposition (deposição de vapor químico térmico rápido)

Si Silício

SiGe Liga de Silício e Germânio

Si $i_{1-x} \mathrm{Ge}_{\mathrm{x}} \quad$ Liga de Silício e Germânio, sendo x fração atômica de germânio na liga

$\mathrm{SiO}_{2} \quad$ Dióxido de Silício.

SOI Silicon-on-Insulator (silício-sobre-isolante)

SRH Shockley-Read-Hall

SS Subtreshold Swing (Inclinação de Sublimiar) 
SSRM Scanning Spreading Resistance Microscopy (microscópio de varredura do espalhamento da resistência)

TAT Trap Assisted Tunneling (tunelamento assistido por armadilhas)

TCAD Technology Computer-Aided Design

TEM Transmission electron microscopy (microscópio eletrônico de transmissão)

TFET Tunnel Field Effect Transistor (transistor de tunelamento por efeito de campo)

TiN Nitreto de Titânio

a-Si Silício amorfo

a-Ge Germânio amorfo 


\section{LISTA DE SIMBOLOS}

AвтBT Parâmetro de ajuste da taxa de geração de tunelamento de banda para banda $\left[\mathrm{cm}^{-1} \mathrm{~V}^{-2} \mathrm{~s}^{-1}\right]$

Av $\quad$ Ganho de tensão de malha aberta em baixa frequência [dB]

Ввтвт Parâmetro de ajuste da taxa de geração de tunelamento de banda para banda $\left[\mathrm{cm}^{-1} \mathrm{~V}^{-2} \mathrm{~s}^{-1}\right]$

Cox Capacitância do óxido de porta $\left[\mathrm{F} / \mathrm{cm}^{2}\right]$

D Diâmetro [nm]

$\mathrm{E}_{\mathrm{A}} \quad$ Energia de ativação $[\mathrm{eV}]$

Ec Nível de energia inferior da banda de condução [eV]

Eg Largura da banda proibida [eV]

$\mathrm{E}_{\mathrm{i}} \quad$ Nível de Fermi intrínseco [eV]

$E_{t} \quad$ Diferença entre o nível de energia da armadilha e o nível intrínseco $[\mathrm{eV}]$

Ev Nível de energia superior da banda de valência [eV]

F Campo elétrico $[\mathrm{V} / \mathrm{cm}]$

f $\quad$ Frequência $[\mathrm{Hz}]$

gd Condutância de saída [S]

gm $\quad$ Transcondutância [S]

$\hbar \quad$ Constante reduzida de Plank $\left[6,626 \times 10^{-34} \mathrm{~J} . \mathrm{s}\right]$

ID $\quad$ Corrente de dreno $[\mathrm{A}]$

IDS $\quad$ Corrente entre dreno e fonte $[A]$

lofF $\quad$ Corrente de desligamento $[A]$

Ion Corrente de funcionamento $[\mathrm{A}]$

i-Si Silício com concentração intrínseca de portadores. 


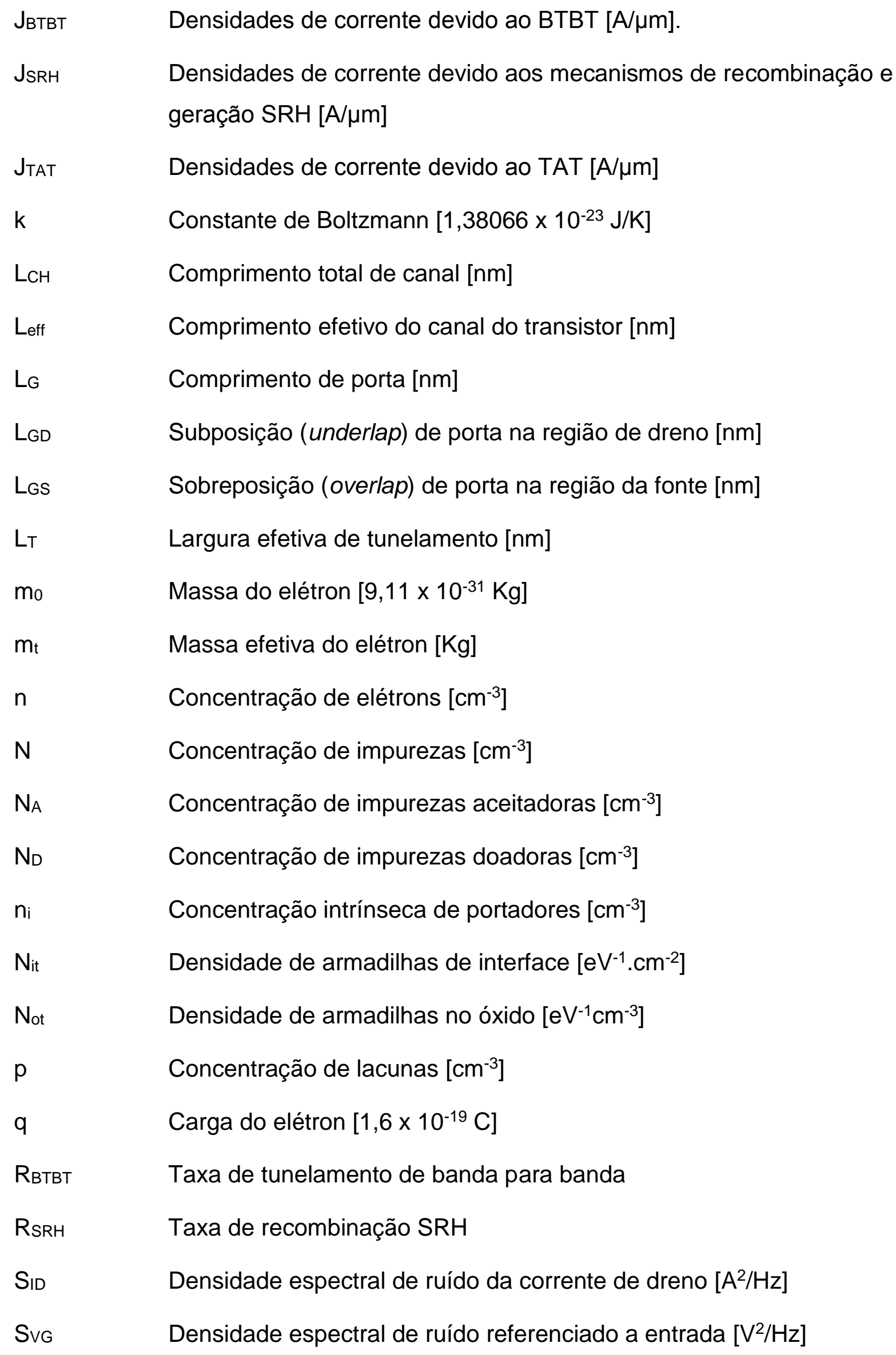


Temperatura $[\mathrm{K}]$

tc-Ge Espessura de Germânio cristalino

$\mathrm{t}_{\mathrm{HFO} 2} \quad$ Espessura de $\mathrm{HfO}_{2}[\mathrm{~nm}]$

VD $\quad$ Tensão aplicada ao dreno [V]

VDS Tensão aplicada entre dreno e fonte [V]

$V_{G} \quad$ Tensão aplicada à porta do transistor [V]

VGS Tensão aplicada entre porta e fonte [V]

Vs $\quad$ Tensão aplicada à fonte [V]

W Largura do canal do transistor [nm]

a Parâmetro de tunelamento $\left[10^{-8} \mathrm{~cm}\right]$

$\Delta V_{G S} \quad$ Variação de $V_{G S}$ na histerese [V]

$\xi \quad$ Campo elétrico $[\mathrm{V} / \mathrm{m}]$

$\tau_{n} \quad$ Tempo de vida das lacunas [s]

$\tau_{p} \quad$ Tempo de vida dos elétrons [s]

$\Gamma_{n}^{T A T} \quad$ Função de efeito de campo para elétrons

$\Gamma_{p}^{T A T} \quad$ Função de efeito de campo para lacunas 


\section{SUMÁRIO}

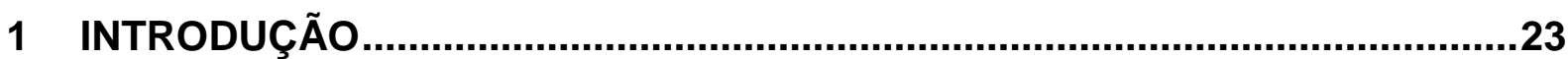

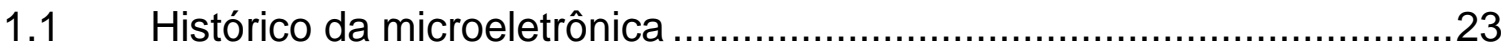

$1.2 \quad$ Histórico dos transistores túnel FET .................................................25

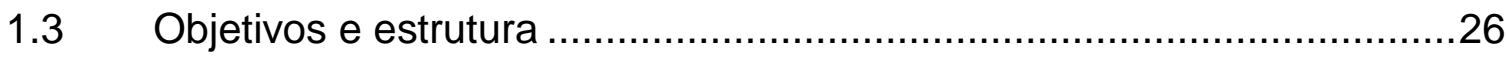

2 TRANSISTORES DE TUNELAMENTO POR EFEITO DE CAMPO...................27

2.1 Modo de funcionamento do TFET ...............................................27

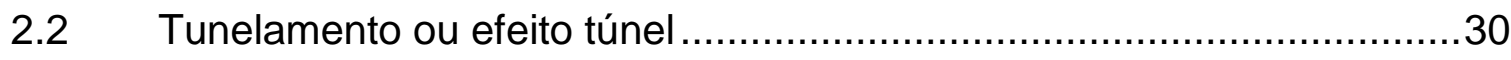

2.3 Mecanismos de condução do TFET ........................................................

2.3.1 Recombinação e geração Shockley-Read-Hall (SRH) ..............31

2.3.2 Tunelamento induzido por armadilhas (TAT) ...........................32

2.3.3 Tunelamento de banda para banda (BTBT) ….........................33

2.3.4 Influência da temperatura nos mecanismos de condução .........34

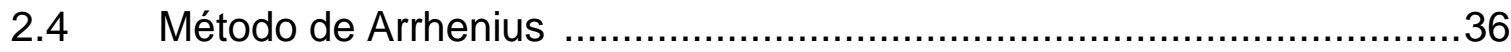

2.5 Ruído em baixa frequência................................................................36

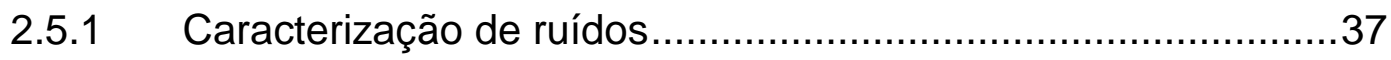

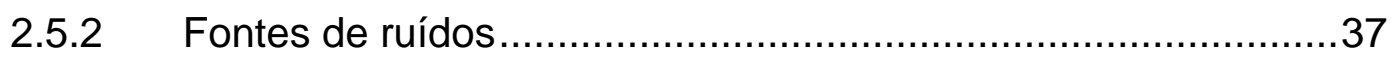

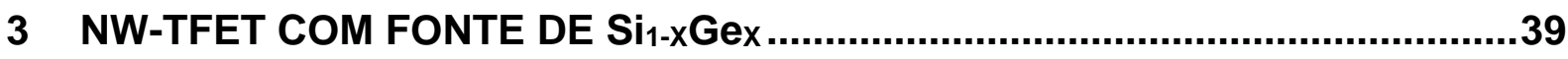

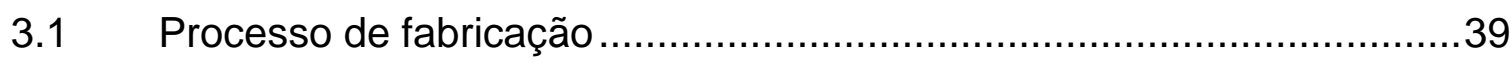

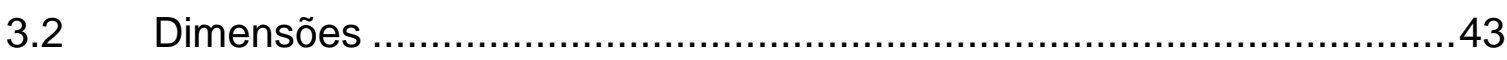

3.3 Estudo do princípio de funcionamento do NW-TFET .............................44

3.4 Influência da temperatura no NW-TFET ...........................................47

3.5 Influência do material de fonte no NW-TFET ……..............................52

3.6 Parâmetros analógicos do NW-TFET com fonte de $\mathrm{Si}_{1-x} \mathrm{Ge}_{\mathrm{x}}$ em função da temperatura

3.7 Influência da densidade de armadilhas de interface para NW-TFET com diferentes materiais de fonte 


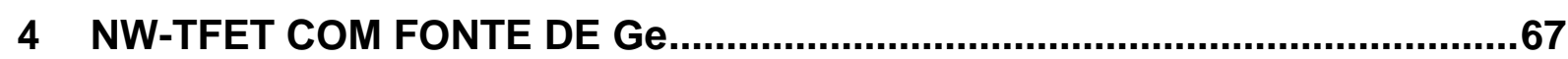

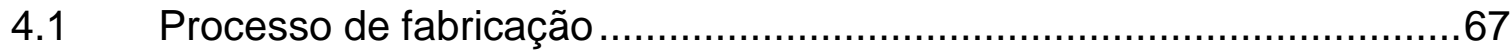

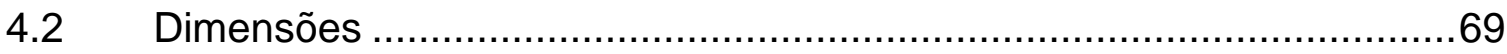

4.3 Parâmetros analógicos do NW-TFET com fonte de Ge e a influência da espessura do óxido de porta .............................................................. 70

4.4 Estudo de ruído de baixa frequência em transistores NW-TFET ............76

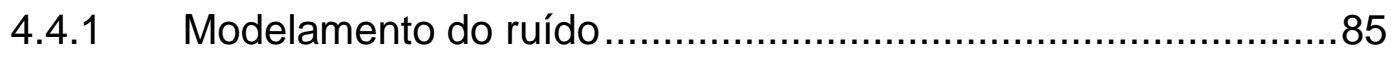

4.4.2 Estudo de ruído em baixa temperatura....................................8

4.5 Estudo de histerese em TFETs com fonte de Ge................................91

5 COMPARAÇÃO DO COMPORTAMENTO ANALÓGICO DOS NW-TFETS COM

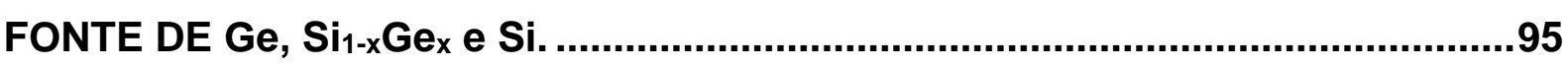

6 CONCLUSÕES E PROPOSTAS DE TRABALHOS FUTUROS .......................100

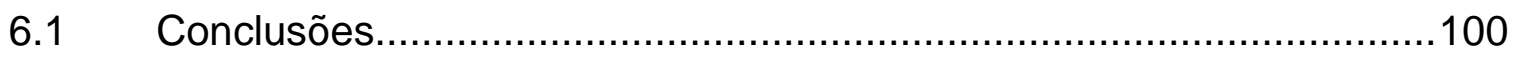

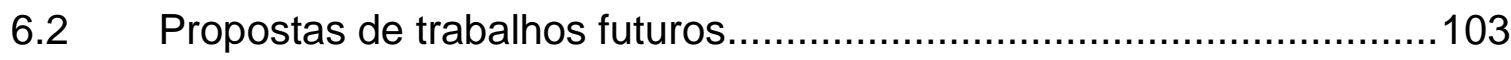

PUBLICAÇÕES GERADAS DURANTE O DOUTORADO .....................................104

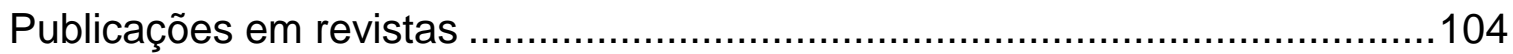

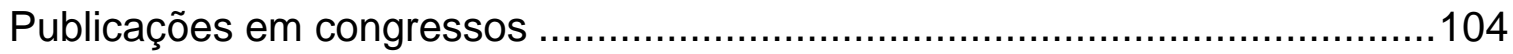

Artigo de revista em processo de submissão..................................................107

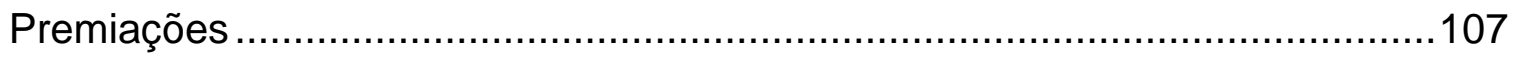

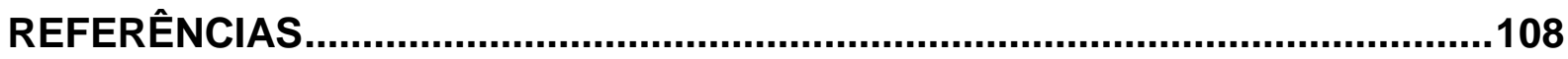

ANEXO A

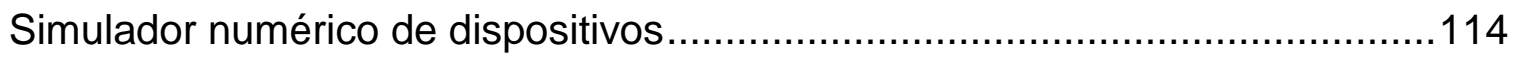

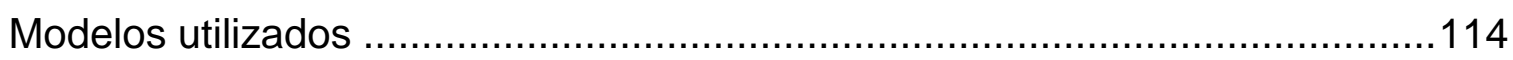

ANEXO B

Exemplo de um script utilizado para gerar uma estrutura em TCAD Sentaurus .....

Exemplo de um script de simulação em TCAD Sentaurus................................121

Exemplo de um script de simulação em TCAD Silvaco ....................................123 


\section{INTRODUÇÃO}

\subsection{Histórico da microeletrônica}

A microeletrônica está relacionada com a tecnologia voltada à produção de circuitos integrados $(\mathrm{Cl})$, promovendo a miniaturização dos componentes eletrônicos. Os circuitos integrados podem englobar bilhões de componentes em uma pequena pastilha de silício, resultando na redução de custo de montagem de sistemas e do volume (1).

O principal componente dos circuitos integrados ao longo das últimas décadas foi o transistor MOSFET (Metal-Oxide-Semiconductor Field-Effect Transistor transistor de efeito de campo metal-óxido-semicondutor). O nome do transistor MOSFET origina-se do seu princípio físico de funcionamento, onde o mecanismo de controle da corrente que flui através do dispositivo é baseado no campo estabelecido pela tensão aplicada no terminal de controle.

Com a miniaturização dos dispositivos MOSFETs surgiram efeitos indesejados, especialmente decorrentes da redução do comprimento de canal, chamados de efeito de canal curto. Com isso, foi incentivado o desenvolvimento de uma nova tecnologia com o intuito de reduzir estes efeitos, dando origem à tecnologia silício sobre isolante (Silicon On Insulator), denominada SOI MOSFET (2).

Apesar dos transistores SOI permitirem uma maior longevidade que os transistores MOSFETs convencionais, o problema do controle das cargas na região de canal em dimensões extremamente reduzidas vem sendo exaustivamente tratado pela comunidade científica mundial na atualidade (2-6).

Tendo em mente a importância de manter esta evolução tecnológica e do escalamento que torna os dispositivos cada vez mais complexos, o estudo de novos materiais, desenvolvimento de novas estruturas e dispositivos com princípios de funcionamentos diferentes vem sendo cada vez mais necessário.

A figura 1.1 apresenta o gráfico da evolução tecnológica ao longo dos últimos anos, apresentando as estruturas mais promissoras a suceder a tecnologia MOSFET. 
Figura 1.1 - Evolução tecnologia ao longo do tempo (7)

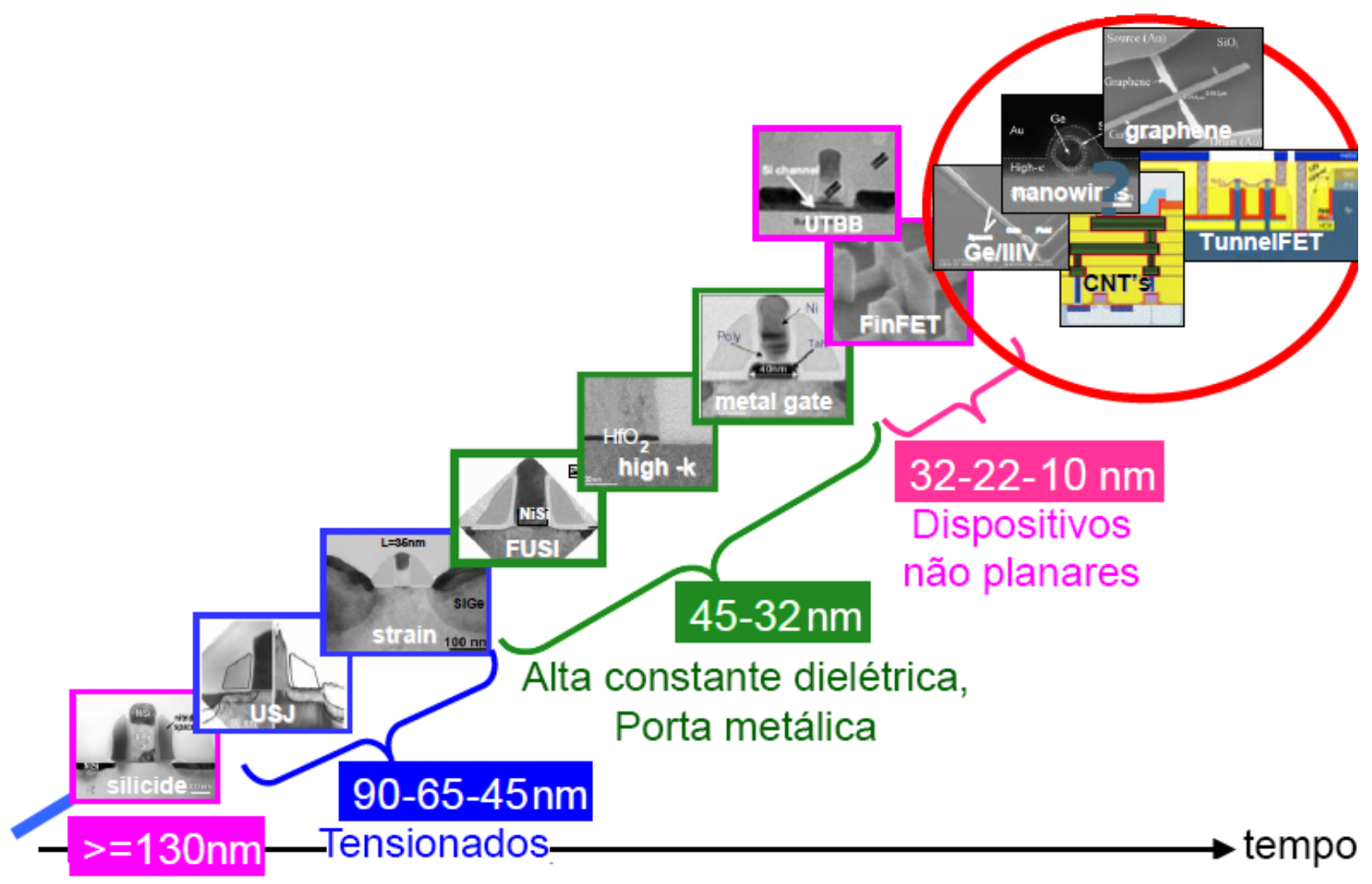

Fonte: Adaptado de Claeys (2009)

Um dos dispositivos considerados mais promissores a dar continuidade ao escalamento e substituir a tecnologia MOSFET em aplicações de baixa potência é o transistor de tunelamento por efeito de campo (Tunnel Field Efect Transitors - TFET) (7-15).

O funcionamento do TFET é baseado nos efeitos de tunelamento quântico, onde o mecanismo de transporte de corrente elétrica deve-se predominantemente ao tunelamento de elétrons induzido pelo campo elétrico (7-15), ao contrário do MOSFET convencional que se deve ao mecanismo de difusão e deriva (2).

Este princípio de condução deve-se ao fato do TFET ser projetado em estrutura do tipo $\mathrm{P}$ - intrínseco - $\mathrm{N}$ controlada por porta, facilitando o tunelamento dos portadores majoritário da região de fonte para o canal.

Devido à corrente de tunelamento ser o mecanismo de transporte predominante no funcionamento do TFET, este dispositivo apresenta diversas vantagens, como potencial de atingir valores de inclinação de sublimiar (Subtreshold Swing - SS) inferiores ao limite físico do MOSFET de $60 \mathrm{mV} / \mathrm{dec}$ em temperatura 
ambiente (11-13), garantindo maior velocidade de chaveamento. Os dispositivos TFET também apresentam corrente de desligamento (loFF) muito baixa, fazendo com que apresente menor potência dissipada quando desligado (16). Além de não apresentar efeitos de canal curto (17).

Embora as características mencionadas levem a uma perspectiva promissora, os TFETs apresentam algumas desvantagens como corrente ambipolar e baixa corrente de funcionamento (lon). Por isso, algumas estratégias vêm sendo estudadas para aperfeiçoar este dispositivo e superar esses problemas (18-23).

\subsection{Histórico dos transistores túnel FET}

O primeiro diodo de tunelamento foi relatado em 1957 por Esaki, utilizando um diodo de germânio com junção P-N altamente dopada (24). Em 1965, Hofstein e Warfield demonstraram o primeiro diodo de tunelamento controlado por porta (25), porém, concluíram que este dispositivo não oferecia vantagens em relação ao MOSFET.

A primeira proposta de um transistor TFET surgiu em 1978 por Quinn et al., onde a estrutura de um transistor MOSFET foi alterada, obtendo uma estrutura do tipo estrutura $\mathrm{P}^{+}-\mathrm{P}^{-}-\mathrm{N}^{+}$com controle de porta (26). Entretanto, este estudo não tem nenhuma relação com as motivações mais recentes, sendo demonstrado apenas a utilidade desse novo transistor em aplicações de espectroscopia.

Em 1987, Banerjee et al., utilizando uma estrutura similar à do Quinn, demonstrou que o TFET apresentava características de saída similares a do MOSFET, exceto pela transcondutância não linear, sugerindo o uso para circuitos analógicos específicos (27).

Posteriormente, surgiram novos trabalhos sobre transistores de tunelamento de banda para banda, porém com diferentes nomenclaturas. Em 1988, Takeda et al. realizaram a caracterização elétrica de um transistor com estrutura do tipo $\mathrm{P}^{+-} \mathrm{N}^{-}-\mathrm{N}^{+} \mathrm{e}$ usaram a denominação B ${ }^{2}$ T- MOSFET (28). Em 1992, Baba propôs um transistor $\mathrm{P}^{+}-\mathrm{I}-\mathrm{N}^{+}$controlados por porta e o nomeou Transistores de Tunelamento Superficial (Surface Tunnel Transistor - STT) (29). Neste estudo o STT foi sugerido como possível substituto do MOSFET devido aos reduzidos efeitos de canal curto. 
A redução da inclinação de sublimiar para valores abaixo de $60 \mathrm{mV} / \mathrm{dec}$ foi reportada pela primeira vez em 2004 por Appenzeller et al., utilizado transistores de nanotubo de carbono (Carbon Nanotube Field Effect Transistor - CNTFET) (11). Assim, abrindo as portas para o TFET como um possível substituto ao MOSFET em circuitos de baixa potência.

Em 2004, Bhuwalka et al. publicaram o primeiro artigo de TFET vertical utilizando SiGe em sua estrutura (12). Neste trabalho foi observado que devido a menor largura da banda proibida do SiGe, houve a redução da barreira de tunelamento e consequentemente, o aumento da corrente de tunelamento e redução da inclinação de sublimiar.

Nos anos seguintes, surgiram novas propostas com variações de estruturas e diferentes condições de polarização (18-23).

\subsection{Objetivos e estrutura}

O objetivo deste trabalho foi estudar detalhadamente a influência de cada mecanismo de transporte em função das diferentes condições de polarização do TFET, a influência da temperatura, a influência dos defeitos de armadilhas de interface

e armadilhas no óxido, decorrentes do processo de fabricação em seu comportamento, caracterização elétrica dos principais parâmetros analógicos, seguido pelo estudo do ruído em baixa frequência e por fim, uma proposta de modelamento de ruído em baixa frequência para o TFET.

Este trabalho está organizado em 6 capítulos, sendo o primeiro este que apresenta um breve histórico e introdução ao tema. No segundo capitulo são apresentados os conceitos teóricos sobre o funcionamento básico dos TFETs e os diferentes mecanismos de condução de portadores envolvidos. Nos capítulos 3 e 4 são apresentados e discutidos resultados decorrentes de medidas experimentais e simulações numéricas para transistores TFETs com fonte de Si, liga de SiGe ou Ge. $\mathrm{Na}$ sequência, serão apresentadas as conclusões do trabalho, propostas para sequência de trabalho, uma lista com todas as publicações geradas até o momento, seguida pelas referências bibliográficas. 


\section{TRANSISTORES DE TUNELAMENTO POR EFEITO DE CAMPO}

Neste capítulo são apresentados os principais conceitos dos Transistores de tunelamento por efeito de campo (TFET), sua estrutura, modo de funcionamento e os principais mecanismos de transporte de portadores.

\subsection{Modo de funcionamento do TFET}

Os dispositivos TFET são baseados na tecnologia do diodo PIN, apresentando junções do tipo $\mathrm{P}^{+}$- Intrínseco - $\mathrm{N}^{+}$com controle de porta (8), conforme pode ser visto na figura 2.1, onde $V_{G}$ é terminal de porta, $V_{s}$ o terminal de fonte e $V_{D} \circ$ terminal de dreno.

Figura 2.1 - Seção transversal de um TFET de porta de simples

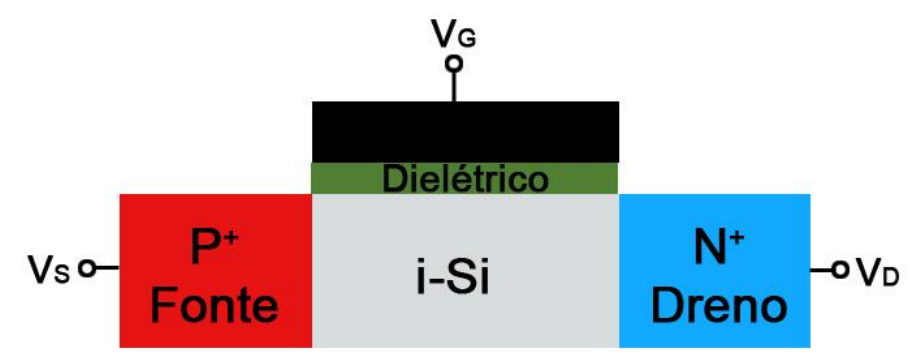

Fonte: Autor

A mesma estrutura TFET pode ser do tipo $\mathrm{p}$ ou $\mathrm{n}$, dependendo das polarizações aplicadas. Em alguns casos para melhorar a corrente de tunelamento do dispositivo, o canal é fracamente dopado com material do tipo N para um nTFET ou com material do tipo $P$ para um pTFET (8).

Assim como na tecnologia MOSFET convencional, a nomenclatura dos terminais de fonte e dreno depende da polarização do dispositivo. Para um nTFET, é considerado uma tensão de porta positiva e polarização reversa das junções P-I-N, dessa forma ocorrendo o tunelamento. A região $\mathrm{N}^{+}$é referida como dreno e a região $\mathrm{P}^{+}$como fonte. De forma análoga, para um pTFET a região $\mathrm{N}^{+}$é referida como fonte e a região $\mathrm{P}^{+}$como dreno. 
O conceito dos dispositivos TFET podem ser implementados utilizando-se de diversos processos de fabricação já existentes, como o de um MOSFET convencional, SOI MOSFET, estruturas de nanofio, estruturas horizontais ou verticais (19). A figura 2.2 apresenta como exemplo a seção transversal de transistores nTFET de múltiplas portas em estrutura horizontal (a) e vertical (b).

Figura 2.2 - Seção transversal de um TFET de múltiplas portas em estrutura horizontal (a) e vertical (b)

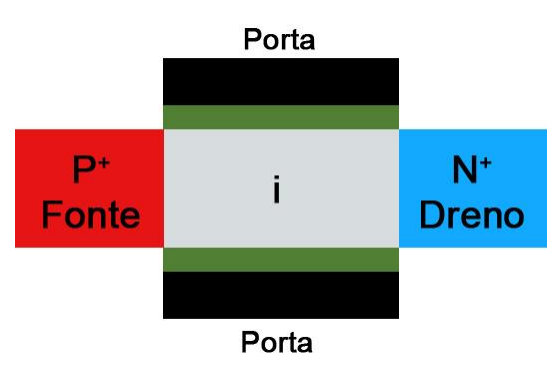

(a)

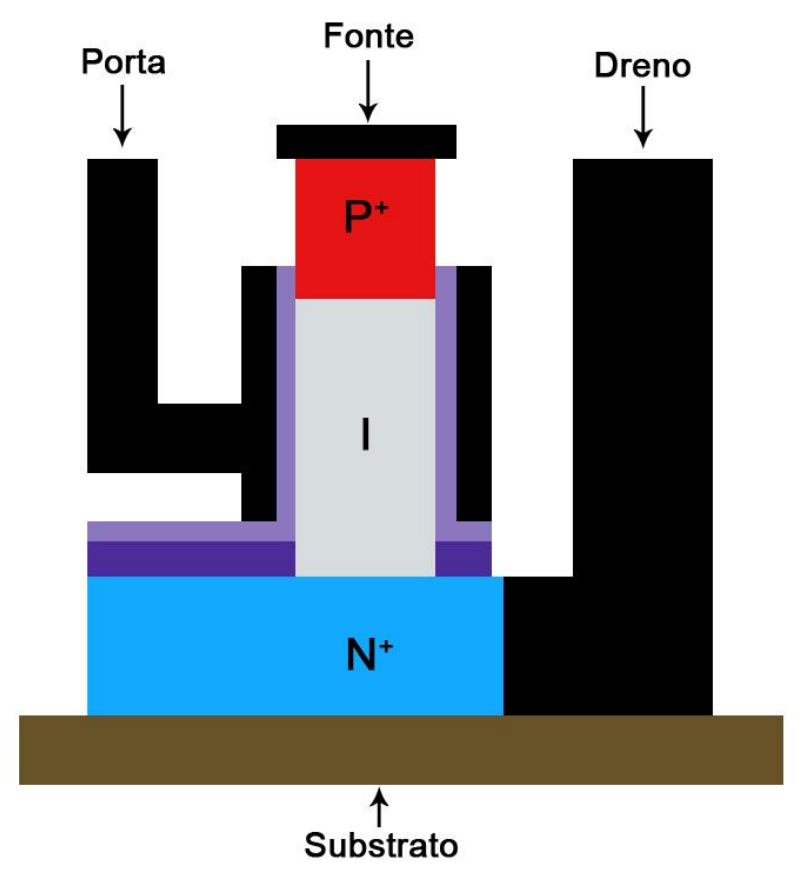

(b)

Fonte: Autor

O princípio de funcionamento do TFET pode ser melhor explicado com a ajuda do diagrama de bandas de energia, apresentado na figura 2.3, onde são exibidos os níveis de energia da banda de valência (Ev) e condução (Ec) das junções $P$-I-N de um nTFET em duas condições de polarização de porta diferentes: com tensão de porta nula (a) e com tensão de porta positiva (b). Para os dois casos está sendo considerado tensão de dreno ( $\left.\mathrm{V}_{\mathrm{DS}}>0 \mathrm{~V}\right)$ positiva.

Quando a tensão de porta é nula ( $\mathrm{V}$ GS $=0 \mathrm{~V})$, o TFET encontra-se em estado desligado, pois a corrente que flui através do dispositivo é muito baixa, sendo devida a recombinação e geração Shockley-Read-Hall $(\mathrm{SRH})$ e a corrente de fuga de junção (16). 
Figura 2.3 - Diagrama de bandas de energia para um nTFET polarizado com tensão de porta nula (a) e tensão de porta positiva (b)

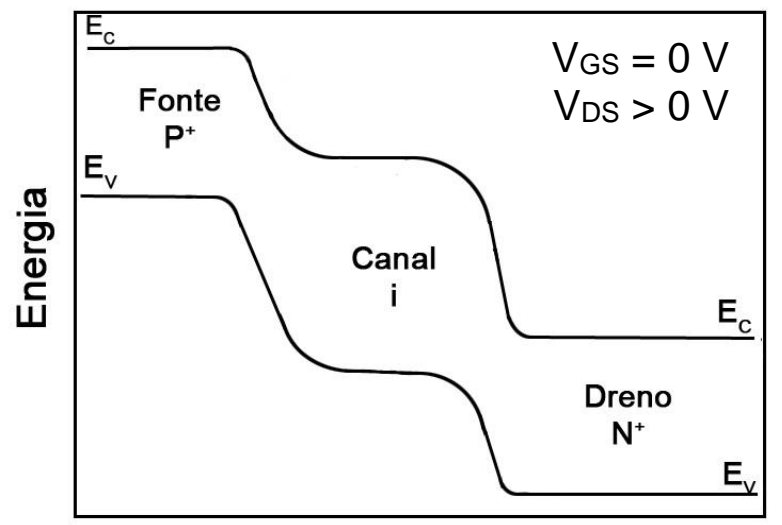

(a)

Posição

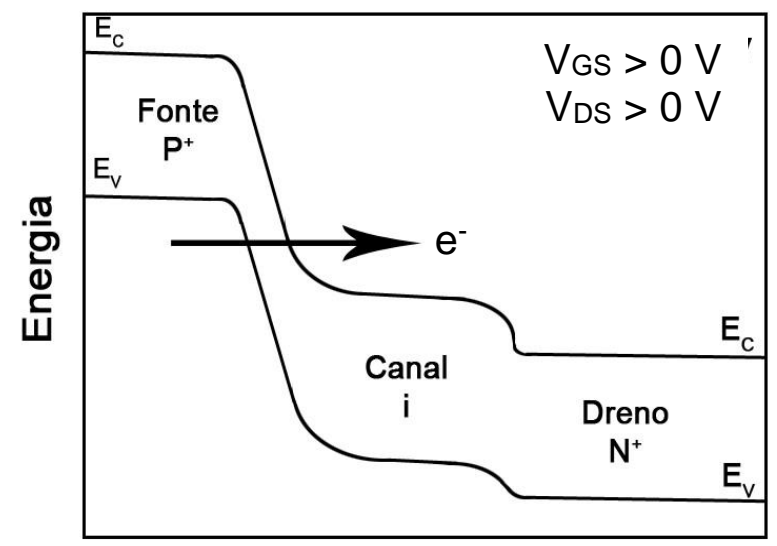

(b)

\section{Posição}

Fonte: Autor

Com tensão de porta positiva ( $\mathrm{VGS}>0 \mathrm{~V}$ ), nota-se na figura 2.3(b) o aumento da barreira de potencial entre a fonte e o canal, fazendo com que o nível máximo da banda de valência na região de fonte fique superior ao nível mínimo da banda de condução na região de canal, viabilizando o tunelamento dos elétrons da banda de valência da fonte para a banda de condução do canal, dando origem à corrente de tunelamento (8).

Quando uma tensão de porta negativa ( $\left.V_{G S}<0\right)$ é aplicada, ocorre o aumento da barreira de potencial entre o dreno e o canal, fazendo com que ocorra 0 tunelamento reverso no dreno, resultando no aumento da corrente de dreno e prejudicando a corrente de desligamento do dispositivo (lofF). Este resultado indesejável é chamado efeito ambipolar (18).

Uma das soluções para que o efeito ambipolar possa ser minimizado ou até mesmo suprimido é fazer com que a porta do dispositivo não esteja auto alinhada com a junção de dreno, criando uma região assimétrica, chamada de subposição entre porta e dreno (gate drain underlap) (18).

O modo de funcionamento detalhado para diversas condições de polarização do TFET é apresentado no capítulo 3.3. 


\subsection{Tunelamento ou efeito túnel}

Segundo a mecânica quântica, um elétron ou uma lacuna possuem probabilidade finita de atravessar uma barreira de potencial mesmo possuindo uma energia menor que a mesma. $O$ valor desta probabilidade, também chamada de coeficiente de transmissão do portador, depende da forma, altura e largura da barreira de potencial (30-32).

Figura 2.4 - Ilha de potencial com barreira de potencial de $2 \mathrm{~nm}$ de largura e $40 \mathrm{mV}$ de altura (a). Função de onda (b) (32)

(a)

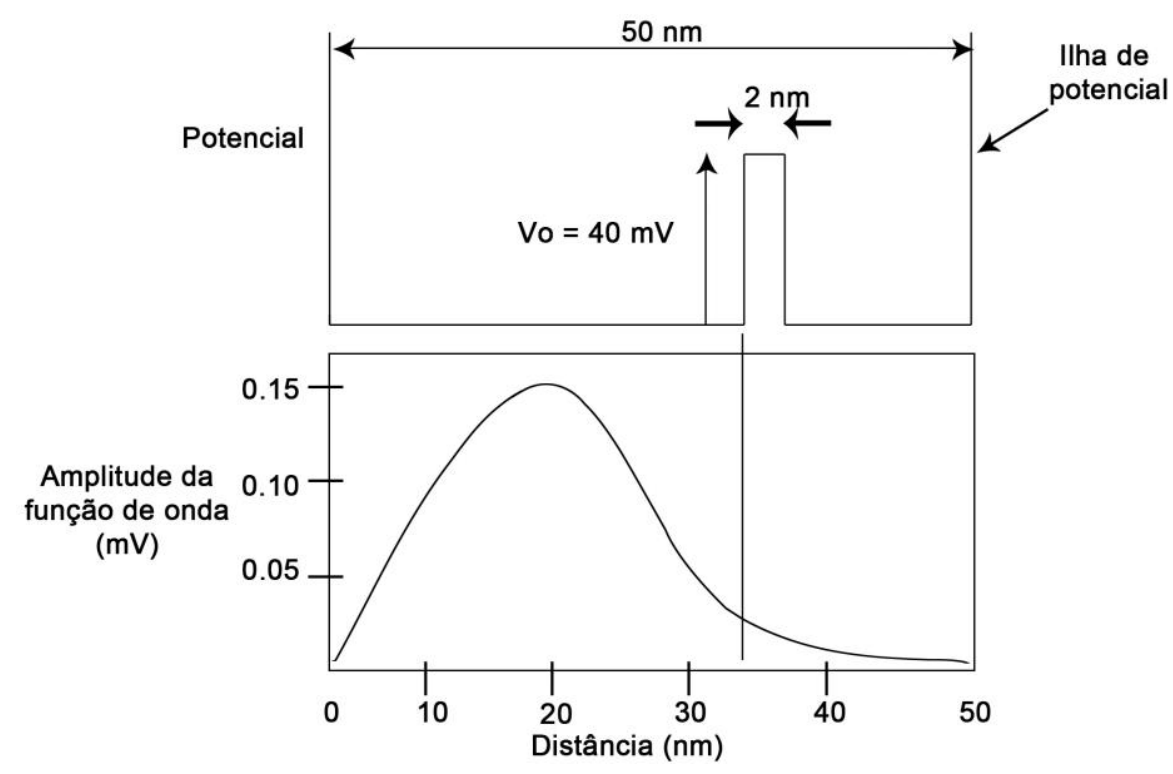

Fonte: Adaptado de Colinge (2002)

Para ilustrar este efeito, considere a figura 2.4(a), onde está apresentado uma ilha de potencial com uma barreira de potencial de $2 \mathrm{~nm}$ de espessura e $40 \mathrm{mV}$ altura. De acordo com a mecânica clássica, se uma partícula estiver posicionada ao lado esquerdo dessa barreira e possui energia superior à altura da mesma, ela terá probabilidade "1" de ser transmitida para o lado direito, caso contrário terá probabilidade "1" de ser totalmente refletida (32).

Porém, segundo a mecânica quântica, as partículas possuem comportamento ondulatório e a função de onda terá valores diferentes de zero dos dois lados da barreira, significando que a partícula poderá estar tanto de um lado quanto do outro 
da barreira. A figura 2.4(b) apresenta esta função onda para um elétron, onde podese observar que o potencial máximo é de $0,15 \mathrm{mV}$, o qual seria insuficiente para vencer a barreira de potencial pela mecânica clássica. Entretanto, de acordo com a mecânica quântica, a função onda possui uma componente capaz de penetrar na barreira, perdendo sua energia exponencialmente com a distância. Quanto mais estreita for esta barreira, maior a probabilidade da partícula ser encontrada do lado direito da barreira de potencial (32). Este fenômeno é chamado de tunelamento ou efeito túnel.

\subsection{Mecanismos de condução do TFET}

Ao longo da análise dos resultados obtidos neste trabalho serão comentadas as influências dos principais mecanismos de condução deste dispositivo, como a recombinação e geração Shockley-Read-Hall ( $\mathrm{g}$-r SRH), o tunelamento induzido por armadilhas (Trap Assisted Tunneling - TAT) e o tunelamento de banda para banda (Band to Band Tunneling - BTBT). Cada uma destas componentes será descrita nos itens a seguir.

\subsubsection{Recombinação e geração Shockley-Read-Hall (SRH)}

A transferência de elétrons entre o nível máximo da banda de valência para o nível mínimo da banda de condução em um material semicondutor não é muito fácil devido a banda de energia proibida (bandgap) ser relativamente elevada, necessitando de uma quantidade de energia muito alta para que isto ocorra. Porém, às impurezas, defeitos na rede cristalina ou contaminações nos semicondutores podem gerar níveis energéticos intermediários de armadilhas na banda proibida. Os pares elétrons-lacunas armadilhados nesta região recombinam-se, dando origem ao efeito de geração Shockley-Read-Hall (SRH) ou multifonons, pois a energia liberada durante o evento de recombinação é dissipada através de vibrações na rede cristalina por meio de fônons (33-34). 
A taxa de recombinação $\mathrm{SRH}(\mathrm{RSRH})$ é dada pela seguinte equação (33):

$$
R_{R S H}=\frac{n \cdot p-n_{i}^{2}}{\tau_{p}\left(n+n_{i} \cdot e^{\left(\frac{E_{t}}{k \cdot T}\right)}\right)+\tau_{n}\left(p+n_{i} \cdot e^{\left(-\frac{E_{t}}{k \cdot T}\right)}\right)}
$$

onde $\mathrm{n}$ é a concentração de elétrons, $\mathrm{p}$ a concentração de lacunas, $\mathrm{n}_{\mathrm{i}}$ a concentração intrínseca de portadores, $\tau_{p}$ o tempo de vida das lacunas, $\tau_{n}$ o tempo de vida dos elétrons, $E_{t}$ a diferença entre o nível de energia da armadilha e o nível intrínseco, $\mathrm{k}$ a constante de Boltzmann e T a temperatura.

A máxima taxa de recombinação e geração $S R H$ é dada quando $E_{t}$ está localizada no meio da banda proibida (34).

\subsubsection{Tunelamento induzido por armadilhas (TAT)}

Assim como a recombinação e geração $\mathrm{SRH}$, o tunelamento induzido por armadilhas (Trap Assisted Tunneling - TAT) ocorre devido ao armadilhamento de elétrons em níveis energéticos intermediários na banda proibida. Com o aumento da barreira de potencial entre a fonte e o canal, torna-se possível o tunelamento dos elétrons armadilhados na região de fonte para a banda de condução do outro lado da junção, na região de canal (35-36).

O modelo de corrente de tunelamento induzido por armadilhas, proposto por Hurkx (36), consiste no acréscimo de um termo denominado função de efeito de campo na equação de recombinação e geração $\mathrm{SRH}$, sendo $\Gamma_{n}^{T A T}$ para elétrons e $\Gamma_{p}^{T A T}$ para lacunas. Reescrevendo a equação 2.1 e acrescentando estes termos: 


$$
R_{T A T}=\frac{n \cdot p-n_{i}^{2}}{\frac{\tau_{p}}{1+\Gamma_{p}^{T A T}}\left(n+n_{i} \cdot e^{\left(\frac{E_{t}}{k \cdot T}\right)}\right)+\frac{\tau_{n}}{1+\Gamma_{n}^{T A T}}\left(p+n_{i} \cdot e^{\left(-\frac{E_{t}}{k \cdot T}\right)}\right)}
$$

sendo:

$$
\begin{aligned}
& \Gamma_{n}^{T A T}=\frac{\Delta E_{n}}{k \cdot T} \int_{0}^{1} e^{\left(\frac{\Delta E_{n}}{k \cdot T} \cdot u-K_{n} \cdot u^{\frac{3}{2}}\right)} d u \\
& \Gamma_{p}^{T A T}=\frac{\Delta E_{p}}{k \cdot T} \int_{0}^{1} e^{\left(\frac{\Delta E_{p}}{k \cdot T} \cdot u-K_{p} \cdot u^{\frac{3}{2}}\right)} d u
\end{aligned}
$$

onde $\Delta \mathrm{E}_{\mathrm{n}}$ e $\Delta \mathrm{E}_{\mathrm{p}}$ são a banda de energia para qual ocorre o tunelamento de elétrons e lacunas, respectivamente, u a variável de integração e $K_{n}$ e $K_{p}$ definidos pelas equações 2.5 e 2.6 .

$$
\begin{aligned}
& K_{n}=\frac{4}{3} \frac{\sqrt{2 \cdot m_{0} \cdot m_{t} \cdot \Delta E_{n}^{3}}}{q \cdot \hbar \cdot|E|} \\
& K_{p}=\frac{4}{3} \frac{\sqrt{2 \cdot m_{0} \cdot m_{t} \cdot \Delta E_{p}^{3}}}{q \cdot \hbar \cdot|E|}
\end{aligned}
$$

onde $\mathrm{m}_{0}$ é a massa do elétron em repouso, $\mathrm{m}_{\mathrm{t}}$ a massa efetiva do elétron, $\hbar$ a constante de Planck reduzida (h/2 $\pi)$.

\subsubsection{Tunelamento de banda para banda (BTBT)}

Devido as características deste dispositivo, quando valores de tensão suficientemente altos são aplicados à porta, o nível superior da banda de valência da região $\mathrm{P}^{+}$(fonte) torna-se maior que o nível inferior da banda de condução da região intrínseca (canal), aumentando muito a probabilidade de tunelamento de banda para banda. Nesta condição de polarização, o tunelamento direto de banda para banda é o principal mecanismo de transporte do TFET (8). 
O modelo de taxa de tunelamento de banda para banda proposto por Hurkx (36) é dado pela equação 2.7.

$$
\begin{gathered}
R_{B T B T}=A_{B T B T} \cdot D \cdot F^{P} \cdot e^{\left(\frac{\left.B_{B T B T \cdot E g \cdot(T)}\right)^{3 / 2}}{E_{g \cdot(300)^{3 / 2} \cdot F}}\right)} \\
D=\frac{n \cdot p-n_{i}^{2}}{\left(n+n_{i}\right)\left(p+n_{i}\right)}
\end{gathered}
$$

onde os coeficiente são $A_{\text {ВтвT }}=4.10^{14} \mathrm{~cm}^{-3} \mathrm{~s}^{-1}, B_{\text {ВTBT }}=1,9.10^{-7} \mathrm{~V} / \mathrm{cm}$ e P $=2,5$, F é a magnitude do campo elétrico.

\subsubsection{Influência da temperatura nos mecanismos de condução}

Para analisar a influência da temperatura em cada um dos mecanismos de condução apresentados, foram obtidas as equações simplificadas da densidade de corrente devido aos mecanismos de recombinação e geração SRH (JSRH), tunelamento assistido por armadilhas (JTAT) e tunelamento de banda para banda (Јвтвт), dado pelas seguintes equações (37):

$$
\begin{aligned}
& J_{R S H} \propto C_{1} \cdot e^{\left(-\frac{E g}{\frac{2}{k}}\right)} \\
& J_{T A T} \propto C_{2} \cdot e^{\left(-\frac{\frac{E_{g}}{2}+\Delta E_{t}}{k \cdot T}\right)} \\
& J_{B T B T} \propto \cdot e^{\left(-C_{3} \cdot \frac{E_{g}^{3 / 2}}{F}\right)}
\end{aligned}
$$


sendo, $\mathrm{C}_{1}, \mathrm{C}_{2}$ e $\mathrm{C}_{3}$ constantes matemática, $\mathrm{E}_{\mathrm{g}}$ a largura de banda proibida, $\Delta \mathrm{E}_{\mathrm{t}}$ a diferença entre o nível de energia da armadilha e o nível intrínseco, $\mathrm{k}$ a constante de Boltzmann, $\mathrm{T}$ a temperatura e $\mathrm{F}$ o campo elétrico.

A partir das simplificações obtidas, podemos observar a dependência direta da temperatura na corrente de recombinação e geração SRH (equação 2.9) e na corrente de tunelamento induzido por armadilhas (equação 2.10), enquanto a corrente de tunelamento de banda para banda (equação 2.11) é influenciada pela temperatura indiretamente devido ao estreitamento da banda proibida com o aumento da temperatura.

A largura da banda proibida para o silício pode ser expressa por (38):

$$
\begin{gathered}
E_{g}=1,17+1,059 \cdot 10^{-5} \cdot T-6,05 \cdot 10^{-7} \cdot T^{2}, \quad \text { para } T \leq 170 \mathrm{~K} \\
E_{g}=1,1785-9,025 \cdot 10^{-5} \cdot T-3,05 \cdot 10^{-7} \cdot T^{2}, \quad \text { para } T>170 \mathrm{~K}
\end{gathered}
$$

A figura 2.5 apresenta a curva da largura de banda proibida em função da temperatura, calculada de acordo com a equação 2.12, onde pode ser visto que, quando a temperatura varia dezenas de graus, a largura de banda proibida varia apenas alguns milésimos.

Figura 2.5 - Curva da $E_{g}$ do Si x T, calculado através da equação 2.12

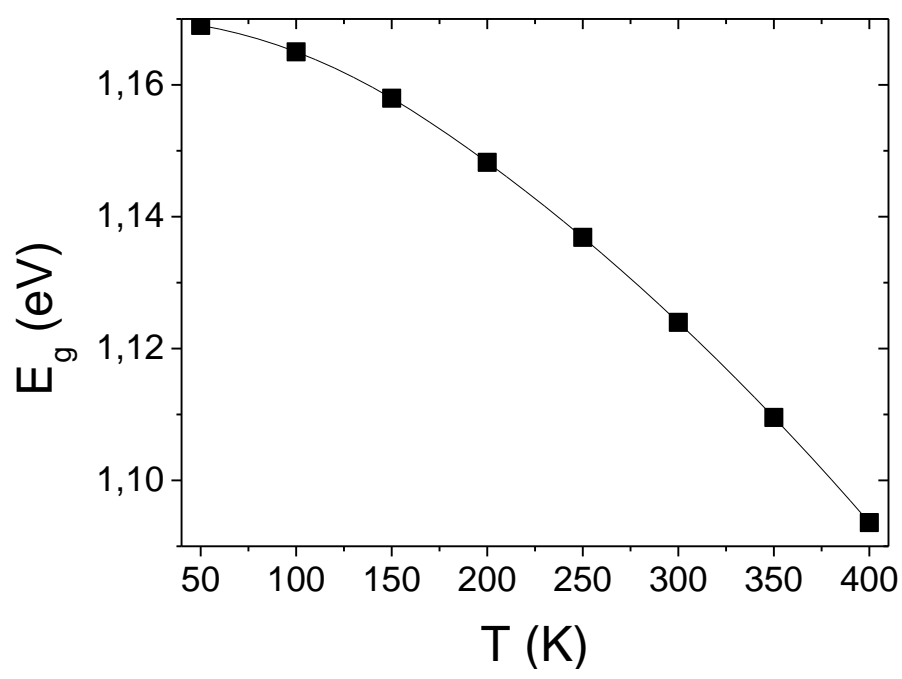

Fonte: Autor 


\subsection{Método de Arrhenius}

O método de Arrhenius (39-40) é um artificio utilizado para determinar qual o mecanismo de condução predominante em diferentes condições de polarização.

Conforme apresentado, cada um dos mecanismos de condução apresentam uma dependência especifica com tensão e temperatura. Através da caracterização elétrica em função da temperatura e utilizando a equação 2.13, é possível calcular a variação de corrente com a temperatura e determinar a energia de ativação $\left(E_{A}\right)$ equivalente.

$$
\ln \left(I_{D S}\right)=-\left(\frac{\boldsymbol{E}_{\boldsymbol{A}}}{K}\right)\left(\frac{1}{T}\right)
$$

Os mecanismos termicamente assistidos, como geração e recombinação $\mathrm{SRH}$ e TAT, tem uma forte sensibilidade à temperatura, apresentando altos valores de $E_{A}$, em torno de $E_{g} / 2$ e $E_{g} / 2+\Delta E_{T}$, respectivamente (41), entretanto, o BTBT apresenta valores tipicamente menores que $0,1 \mathrm{eV}$.

\subsection{Ruído em baixa frequência}

O ruído em um dispositivo eletrônico é uma perturbação aleatória e espontânea de um sinal determinístico inerente a física do dispositivo. Esses distúrbios podem ser originados de fontes externas ao sistema, como linhas de transmissão AC, rádios transmissores ou circuitos adjacentes, perturbando o circuito de interesse devido aos acoplamentos eletrostáticos e eletromagnéticos. Entretanto, o ruído também pode ser de origem interna, quando flutuações randômicas acontecem na estrutura física do material. Esse tipo de ruído não pode ser eliminado, dessa forma, o sinal desejado torna-se difícil de ser distinguido quando a potência do ruído é significativa alta em relação ao sinal, limitando a precisão das medidas e determinando o valor mínimo dos sinais que serão detectados (42). Sendo assim, o ruído é um parâmetro importante a ser compreendido e caracterizado. 


\subsubsection{Caracterização de ruídos}

Um método muito utilizado na literatura para caracterizar o ruído ocorre através da análise da densidade espectral de potência (Power Spectral Density-PSD), obtida através de um analisador de espectro, dando informação de como a potência do ruído é distribuída em função da frequência, a sua unidade é $\mathrm{W} / \mathrm{Hz}$ (42).

O PSD também é comumente expresso nas unidades $A^{2} / \mathrm{Hz}$, representando o PSD de ruído da corrente de dreno (current noise power spectral density - SID) e $\mathrm{V}^{2} / \mathrm{Hz}$, representando a densidade espectral de ruído referenciado a entrada (input referred noise power spectral density - SvG).

\subsubsection{Fontes de ruídos}

Os mecanismos de transporte de portadores através do canal de um transistor é um processo estocástico e apresentam flutuações randômicas, dando origem a diferentes tipos de ruídos, sendo eles (42):

- Ruído térmico ou ruído Johnson-Nyquist:

Este ruído decorre da agitação térmica dos elétrons no interior de um condutor elétrico. $O$ ruído térmico existe em todo resistor e parte resistiva de um dispositivo e corresponde ao menor limite de ruído em um circuito elétrico (42).

- Ruído de barreira ou ruído shot:

Este ruído está associado com a corrente que flui através de uma barreira de potencial, como uma junção p-n, quando os elétrons atravessam essa barreira de forma independente e ao acaso (42).

O ruído térmico e o ruído de barreira apresentam o PSD de ruído constante ao longo de todo o espectro de frequência e por analogia a luz branca que contém todas as frequências do espectro visível, recebem o nome de ruído branco (white noise). 
- Ruido de geração e recombinação (g-r) ou Lorentzian:

O ruído g-r é causado por armadilhas na banda proibida que aprisionam ou emitem portadores aleatoriamente, causando flutuações no número de portadores disponíveis para o transporte de corrente (42). O PSD do ruído g-r em um MOSFET segue o modelo de Lorentz, sendo constante para baixa frequência e sofrendo uma redução com o aumento $f$ em uma taxa de $1 / f^{2}$.

- Ruído Flicker ou ruído 1/f:

O PSD do ruído flicker apresenta uma tendência de $1 / \mathrm{fv}^{\mathrm{v}}$, com $\mathrm{y}$ usualmente no intervalo de 0,7 a 1,3 (42). Este ruído é modelado por dois diferentes mecanismos em um transistor MOSFET. O primeiro deles, proposto por McWhorter (43), considerando que as flutuações na corrente acontecem devido ao armadilhamento e desarmadilhamento das cargas no óxido de porta. Enquanto o segundo, proposto por Hooge (44), considera que o ruído $1 / f$ é devido a flutuações na mobilidade.

A figura 2.6 apresenta o esquemático da densidade espectral de potência (PSD) do ruído na corrente de dreno ( $\mathrm{SID}$ ) em função da frequência (f) para um transistor MOSFET.

Figura 2.6 - Esquemático do SID em função de f para um transistor MOSFET (42)

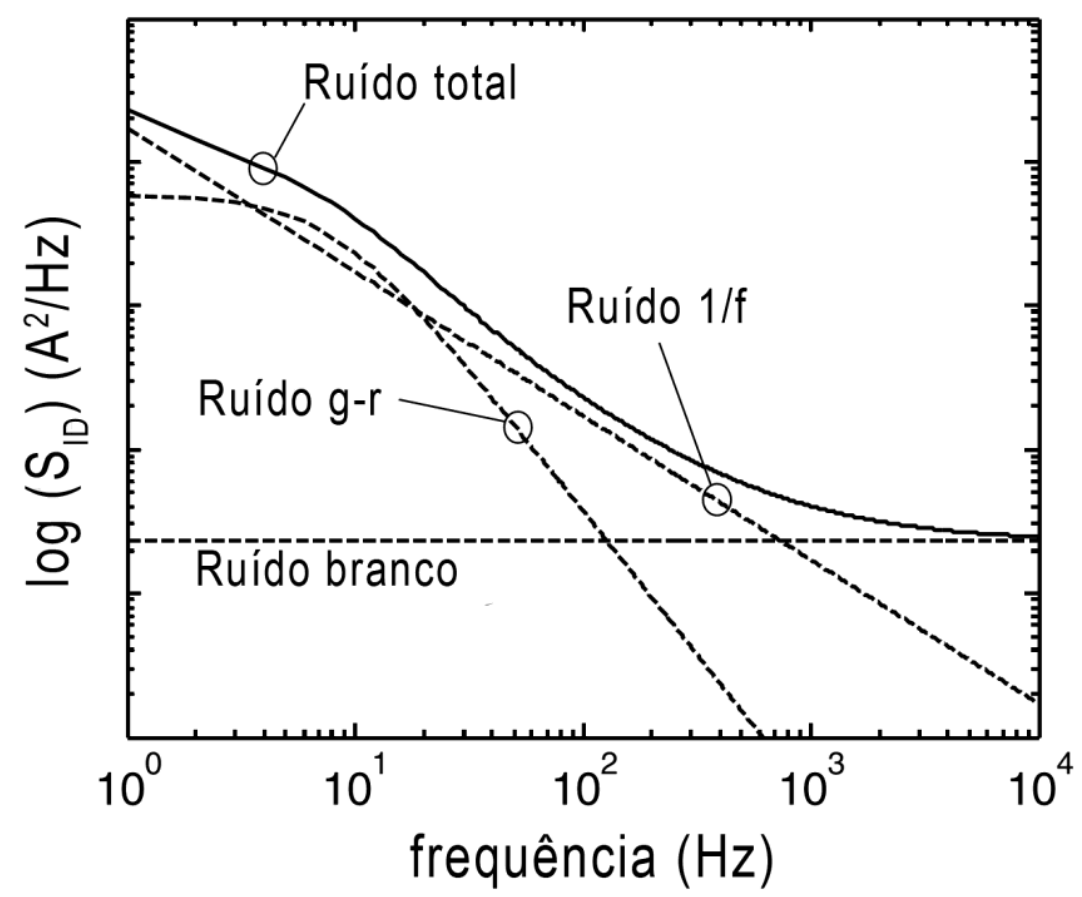

Fonte: Adaptado de Haartman (2007) 


\section{$3 \quad$ NW-TFET COM FONTE DE Si ${ }_{1-x} G e x$}

Recentemente, os transistores TFET atraíram muita atenção devido ao seu rápido chaveamento (SS < $60 \mathrm{mV} / \mathrm{dec}$ ). Entretanto, tem-se reportado que a performance dos TFETs de silício são piores quando comparado aos MOSFETs com baixa corrente de funcionamento, na faixa de 0,1 a $1 \mu \mathrm{A} / \mu \mathrm{m}$ e inclinação de sublimiar (SS) tipicamente entre 100 e $150 \mathrm{mV} /$ dec. Como solução proposta estão a melhora do acoplamento eletrostático com o uso de diferentes materiais de dielétrico de porta, uso de materiais com baixos valores de $E_{g}$ na fonte e otimização das junções, deixando-as mais abruptas (45-49).

Ao longo deste capítulo são apresentados alguns detalhes do processo de fabricação dos TFETs em estruturas de nanofio vertical com fonte de SiGe, o estudo do comportamento destes dispositivos e as vantagens propiciada devido ao uso de SiGe na região de fonte. Para isso, são apresentados resultados experimentais e simulados, iniciando pelo estudo do comportamento dos NW-TFET, seguido pelo estudo da influência da temperatura e das armadilhas de interface em seu funcionamento.

\subsection{Processo de fabricação}

Para realização deste trabalho foram utilizados transistores de tunelamento controlados por efeito de campo em estruturas de nanofio vertical (vertical NW-TFET) fabricados pelo Interuniversity Microelectronics Centre - Imec, Leuven, Bélgica.

A figura 3.1 ilustra algumas etapas do processo de fabricação realizado no Imec, Bélgica (22), iniciando com um substrato de silício altamente dopado com arsênio (material do tipo $\mathrm{N}^{+}$). Em seguida, para a formação da região de canal, é realizado o crescimento epitaxial de uma camada de silício não dopada ou levemente dopada com material do tipo $\mathrm{N}$ com concentração de $10^{15} \mathrm{~cm}^{-3}$. A região de fonte é formada através do crescimento epitaxial de silício, conforme ilustrado na figura 3.1(a). Uma variação deste processo consiste no crescimento epitaxial de uma fina camada de liga $\mathrm{Si}_{1-\mathrm{x}} \mathrm{Ge}_{\mathrm{x}}$, sendo x a porcentagem de germânio na liga, com espessura de algumas dezenas de nanômetros seguida pelo crescimento epitaxial de silício. Esta camada de liga $\mathrm{Si}_{1-\mathrm{x}} \mathrm{Ge}_{\mathrm{x}}$ induz a redução da banda proibida, aumentando a taxa de 
tunelamento e consequentemente, aumentando a corrente de dreno (47). Para reduzir os defeitos apenas alguns nanômetros são construídos em liga $\mathrm{Si}_{1-x} \mathrm{Ge}_{\mathrm{x}}$.

A estrutura de nanofio (nanowire - NW) é obtida através do método de litografia por feixe de elétrons (e-beam litography), figura 3.1(b). Em seguida é depositado o óxido inferior, criando um isolamento entre a porta e o substrato para reduzir a corrente de fuga de porta e a capacitância porta-substrato, além de criar o subposicionamento (underlap) da porta na região de dreno, ilustrado na figura 3.1(c). 0 subposicionamento de porta na região de dreno diminui o tunelamento na interface canal/dreno, reduzindo ou suprimindo a corrente ambipolar $(18,46)$.

Para a formação da região de porta, foi crescido termicamente uma fina camada de óxido de silício $\left(\mathrm{SiO}_{2}\right)$, seguido pela deposição de óxido de háfnio $\left(\mathrm{HfO}_{2}\right)$, um material isolante com alta constante dielétrica (high-k), resultando no aumento da taxa de tunelamento, elevando a corrente de dreno $(47,48)$. A qualidade da interface $\mathrm{Si} / \mathrm{HfO}_{2}$ não é boa, por isso, é necessário a presença de uma fina camada de óxido de silício. Para o metal de porta foi utilizado o nitreto de titânio (TiN). E por fim, depositado Si amorfo a fim de encapsular a camada de TiN e evitar a contaminação nas etapas seguintes do processo, permitindo o auto alinhamento ou a sobreposição (overlap) de porta na região de fonte, figura 3.1(d).

Uma segunda camada de óxido é depositada, figura 3.1(e). As camadas dos materiais de porta expostas no topo da estrutura são corroídas e em seguida é realizada a implantação de boro (material do tipo $\mathrm{P}^{+}$) na região da fonte, conforme apresentado na figura 3.1(f). O contato de fonte é isolado da porta por um espaçador de nitreto, figura 3.1(g). E finalmente são abertas as regiões de contatos do dispositivo, ilustrado na figura $3.1(\mathrm{~h})$. 
Figura 3.1 - Etapas do processo de fabricação de um vertical NW-TFET com fonte de $\mathrm{Si}_{1-x} \mathrm{Ge}_{\mathrm{x}}$ (22)

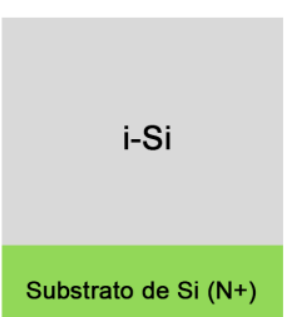

(a)

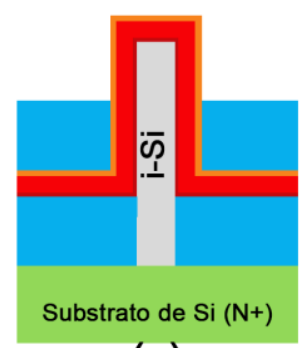

(e)

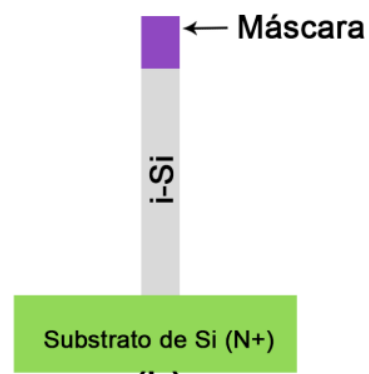

(b)

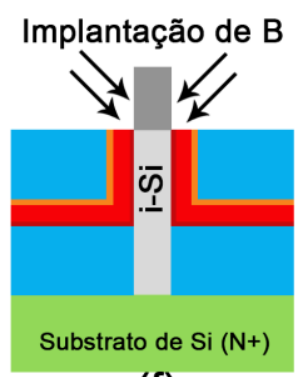

(f)

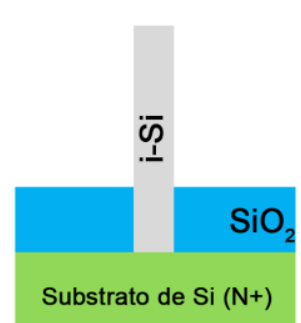

(c)

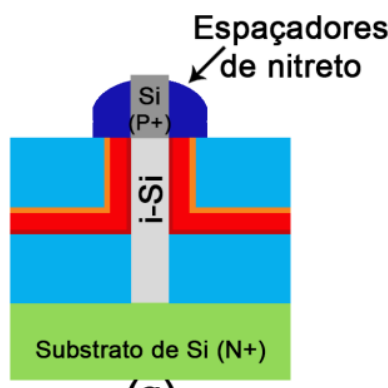

(g)

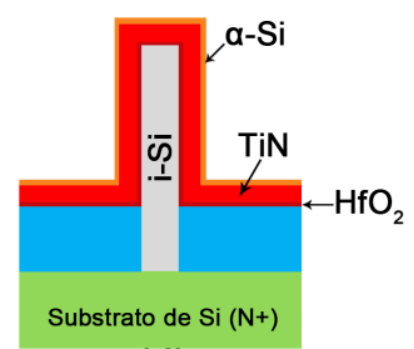

(d)

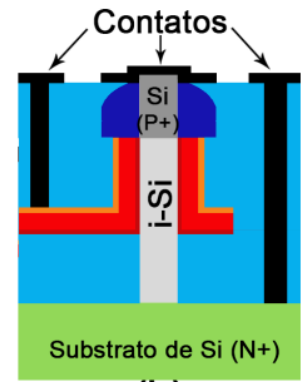

(h)

Fonte: Adaptado de Leonelli (2011)

A figura 3.2(a) apresenta o esquemático da seção transversal de um NW-TFET vertical e a figura 3.2(b) o corte transversal de um dos dispositivos fabricados no Imec, Bélgica obtido em um microscópio eletrônico de transmissão (Transmission electron microscopy - TEM) (50), onde (1) óxido inferior, (2) dielétrico de porta, (3) segundo óxido de isolamento, (4) Isolamento entre fonte e porta (óxido espaçador) e (5) siliceto.

Devido à baixa temperatura de recozimento, todas as dopagens são aproximadamente abruptas. A figura 3.3 apresenta a concentração de dopantes obtida através de um microscópio de varredura da resistência de espalhamento (scanning spreading resistance microscopy - SSRM) (50). 
Figura 3.2 - Esquemático da seção transversal (a) e o corte transversal (b) de um de um NW-TFET vertical

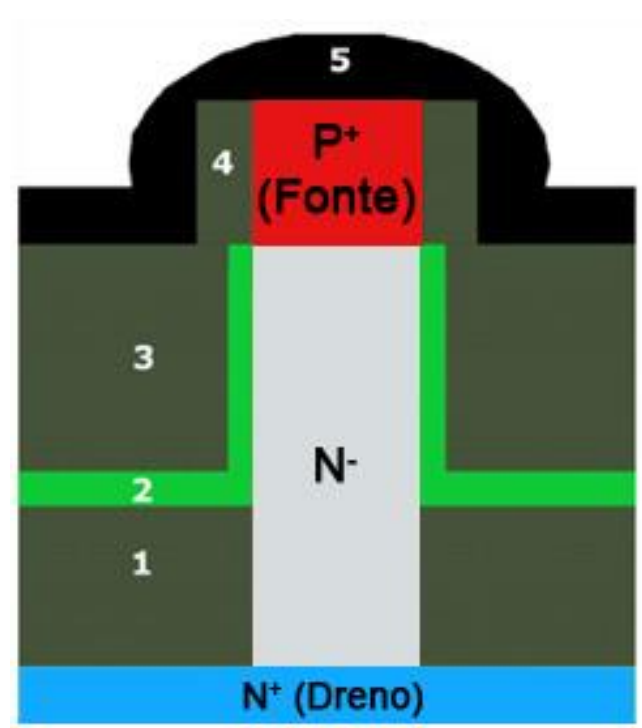

(a)

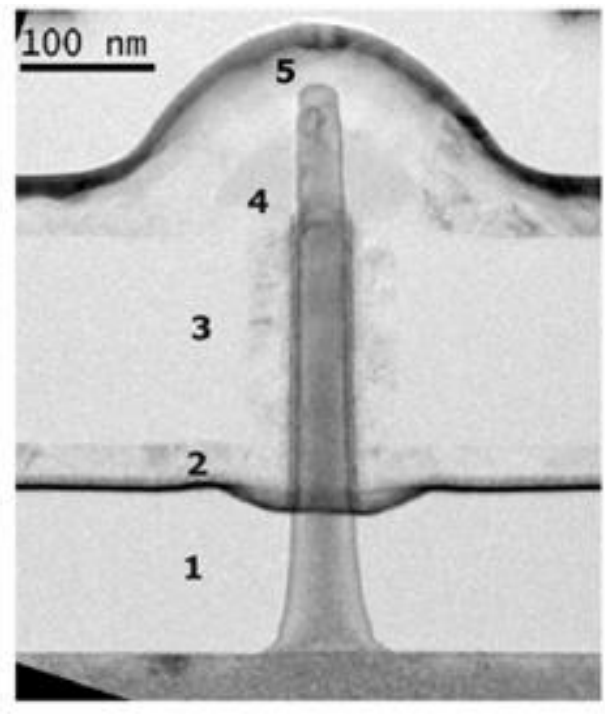

(b)

Fonte: Adaptado de Schulze (2011)

Figura 3.3 - Concentração de dopantes de um NW-TFET (50)
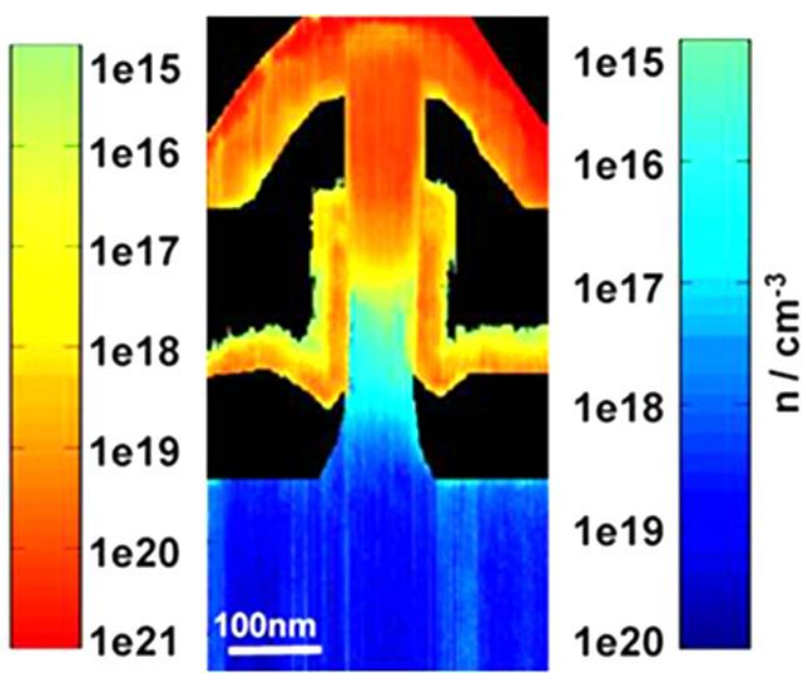

Fonte: Schulze (2011) 


\subsection{Dimensões}

A figura 3.4 ilustra a seção transversal de um NW-TFET indicando o comprimento total de canal ( $\mathrm{LcH}$ ), comprimento de porta $(\mathrm{LG})$, sobreposição (overlap) de porta na região da fonte (LGS), subposição (underlap) de porta na região de dreno (LGD) e diâmetro (D).

Figura 3.4 - Seção transversal de um NW-TFET indicando suas dimensões

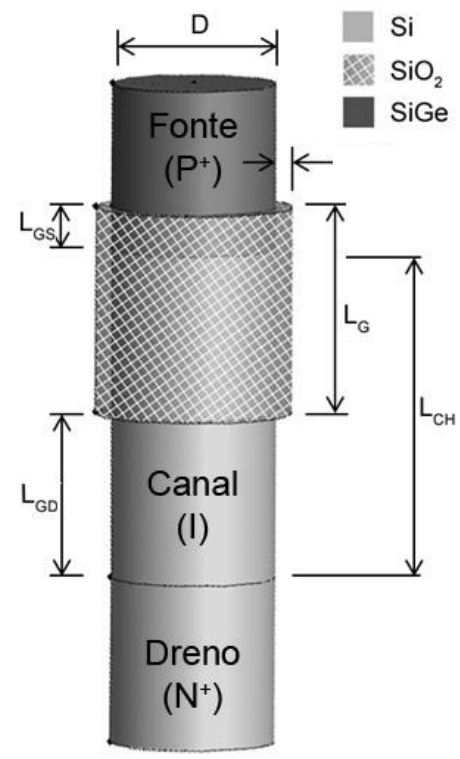

Fonte: Autor

Os resultados experimentais foram obtidos utilizando quatro lâminas de dispositivos diferentes, sendo a Lâmina 1 fabricada em 2011 no primeiro processo de NW-TFET vertical realizado no Imec, Bélgica e as lâminas 2, 3 e 4 em 2012, em uma segunda rodada de fabricação. A tabela 3.1 apresenta as dimensões de cada uma das lâminas utilizadas neste trabalho.

Todas as estruturas apresentam sobreposição da porta na região de fonte, fazendo com que tensões positivas de porta reduzam ainda mais o estreitamento da banda proibida, resultando no aumento da corrente de tunelamento. Todas as estruturas também apresentam subposição da porta na região de dreno, para diminuir ou suprimir a corrente ambipolar.

Além disso, foram utilizadas cascatas com 20 ou 100 transistores em paralelo (NWs), com o intuito de obter valores mais altos de corrente de funcionamento dos dispositivos. 
Tabela 3-1 - Dimensões dos dispositivos NW-TFET fabricados no Imec, Bélgica e utilizados neste trabalho

\begin{tabular}{|c|c|c|c|c|}
\hline & Lâmina 1 & Lâmina 2 & Lâmina 3 & Lâmina 4 \\
\hline Material da fonte & $\mathrm{Si}_{0,73} \mathrm{Ge}_{0,27}$ & $\mathrm{Si}_{0,73} \mathrm{Ge}_{0,27}$ & $\mathrm{Si}_{0,54} \mathrm{Ge}_{0,46}$ & $\mathrm{Si}$ \\
\hline Comprimento de canal ( $\mathrm{LCH})$ & $200 \mathrm{~nm}$ & \multicolumn{3}{|c|}{$160 \mathrm{~nm}$} \\
\hline Comprimento de porta $\left(\mathrm{L}_{\mathrm{G}}\right)$ & $150 \mathrm{~nm}$ & \multicolumn{3}{|c|}{$150 \mathrm{~nm}$} \\
\hline Sobreposição da porta/fonte (LGS) & $30 \mathrm{~nm}$ & \multicolumn{3}{|c|}{$30 \mathrm{~nm}$} \\
\hline Subposição de porta/dreno (LGD) & $100 \mathrm{~nm}$ & \multicolumn{3}{|c|}{$40 \mathrm{~nm}$} \\
\hline Dopagem de fonte & $1.10^{20} \mathrm{~cm}^{-3}$ & \multicolumn{3}{|c|}{$1.10^{20} \mathrm{~cm}^{-3}$} \\
\hline Dopagem de canal & $5.10^{15} \mathrm{~cm}^{-3}$ & \multicolumn{3}{|c|}{$1.10^{15} \mathrm{~cm}^{-3}$} \\
\hline Dopagem de dreno & $5.10^{19} \mathrm{~cm}^{-3}$ & \multicolumn{3}{|c|}{$1.10^{20} \mathrm{~cm}^{-3}$} \\
\hline Espessura equivalente de óxido (EOT) & $1,6 \mathrm{~nm}$ & \multicolumn{3}{|c|}{$3 \mathrm{~nm}$} \\
\hline Diâmetro (D) & $\begin{array}{c}98,160 \mathrm{e} \\
213 \mathrm{~nm}\end{array}$ & \multicolumn{3}{|c|}{$200 \mathrm{~nm}$} \\
\hline Nanofios em paralelo (NWs) & 20 & \multicolumn{3}{|c|}{100} \\
\hline
\end{tabular}

Fonte: Autor

\subsection{Estudo do princípio de funcionamento do NW-TFET}

Nesta primeira etapa do trabalho, a fim de melhor entender o princípio de funcionamento e determinar os principais mecanismos de condução em cada polarização do NW-TFET foram realizadas simulações numéricas tridimensionais no TCAD Sentaurus, utilizando estruturas com dimensões e características similares às da lâmina 1, apresentadas no capítulo 3.2, sendo: comprimento total de canal $\left(\mathrm{L}_{\mathrm{CH}}\right)$ de $200 \mathrm{~nm}$, comprimento de porta $(\mathrm{LG})$ de $150 \mathrm{~nm}$, sobreposição de porta na região da fonte (LGS) de $30 \mathrm{~nm}$, subposição de porta na região de dreno (LGD) de $100 \mathrm{~nm}$, espessura equivalente de óxido (EOT) de 1,6 nm e diâmetro (D) de $200 \mathrm{~nm}$. Região de fonte dopada com $\mathrm{N}_{\mathrm{A}}=1.10^{20} \mathrm{~cm}^{-3}$, canal com $\mathrm{ND}_{\mathrm{D}}=5.10^{15} \mathrm{~cm}^{-3}$ e dreno com $\mathrm{N}_{\mathrm{D}}=$ $5.10^{19} \mathrm{~cm}^{-3}$. Fonte projetada com liga $\mathrm{Si}_{0,73} \mathrm{Ge}_{0,27}$.

Foram obtidas nestas simulações as características da corrente de dreno em função da tensão aplicada à porta (IDS $\times V_{G S S}$ ), com tensão de dreno de $0.9 \mathrm{~V}$, demonstradas na figura 3.5 indicando qual dos diferentes mecanismos de condução predomina em cada região da curva. E os diagramas de bandas de energia para as regiões de fonte, dreno e canal com diferentes tensões de porta ( $\left(\mathrm{VGS}_{\mathrm{G}}\right)$, obtidos a partir da mesma simulação, apresentados na figura 3.6. 
Baseado no diagrama de bandas de energia da figura 3.6(a) é possível observar que para tensões de porta inferiores a 0,5 V há uma considerável barreira de energia separando as bandas de valência da fonte e a banda de condução do canal, dificultando o tunelamento. Nesta condição, o transistor está em estado desligado (OFF State) e a baixa corrente de dreno deve-se à corrente de fuga das junções e da recombinação e geração Shockley-Read-Hall (SRH).

Com o aumento da tensão de porta, observa-se na figura 3.6(b) que para valores próximos a $0,5 \mathrm{~V}$ ocorre o aumento da barreira de potencial, resultando na diminuição da diferença entre o nível máximo da banda de valência da fonte e o nível mínimo da banda de condução do canal, dando início ao tunelamento induzido por armadilhas. Nestas simulações, as armadilhas foram consideradas no centro da banda proibida $\left(E_{t}=0 \mathrm{~V}\right)$.

Figura 3.5 - Curva de los $x$ VGs para um NW-TFET com fonte de $\mathrm{Si}_{0,73} \mathrm{Ge}_{0,27}$ simulada no TCAD Sentaurus

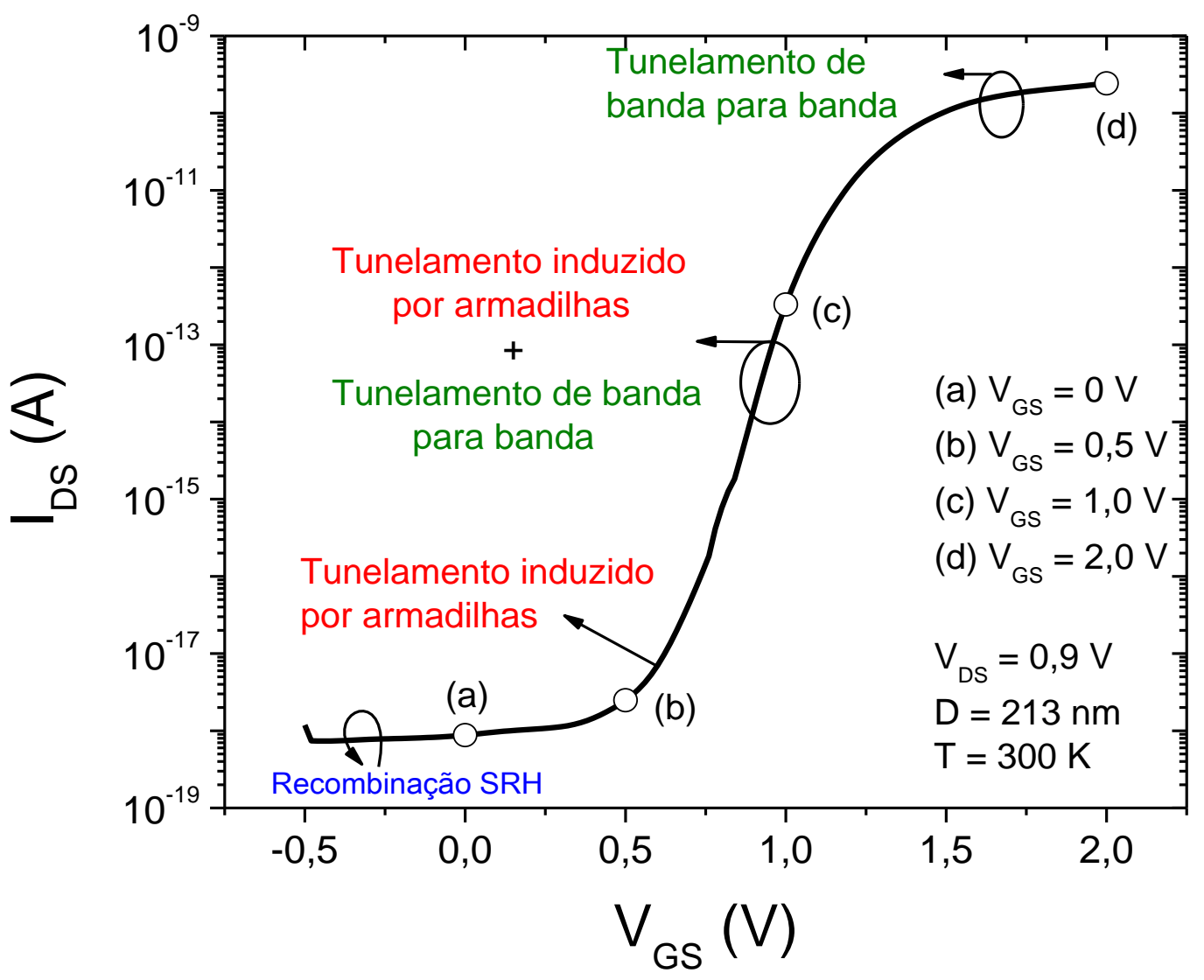

Fonte: Autor 
Figura 3.6 - Diagramas de bandas de energia para um NW-TFET com fonte de $\mathrm{Si}_{0,73} \mathrm{Ge}_{0,27} \mathrm{em}$ diferentes condições de polarização, obtido por simulações no TCAD Sentaurus
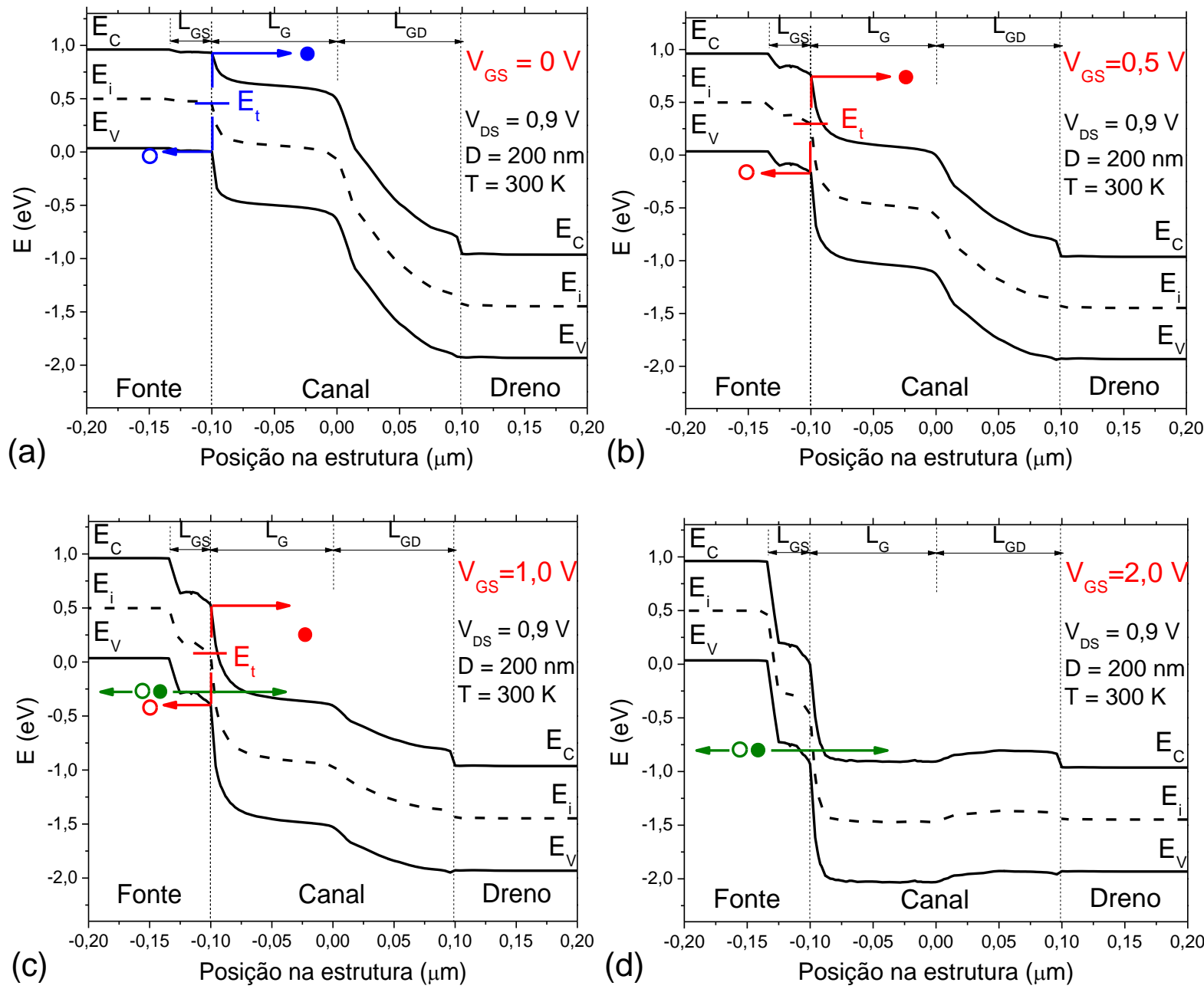

Fonte: Autor

Aumentando um pouco mais a tensão de porta, para valores próximos à $1 \mathrm{~V}$, nota-se na figura 3.6(c) que na interface fonte/canal o ponto máximo da banda de valência e o ponto mínimo da banda de condução estão quase alinhados, fazendo com que comece a ocorrer o tunelamento de banda para banda. Nesta condição a taxa de tunelamento induzidos por armadilhas e a taxa de tunelamento de banda para banda são muito próximas.

Finalmente, para valores de tensão de porta mais altos e consequentemente maior aumento da barreira de potencial, o ponto máximo da banda de valência da fonte torna-se maior que o ponto mínimo da banda de condução do canal, resultando no tunelamento direto de banda para banda, fazendo com que este seja o principal mecanismo de transporte do dispositivo, conforme demonstrado na figura 3.6(d). 


\subsection{Influência da temperatura no NW-TFET}

Paralelamente aos estudos anteriores, a partir da lâmina 2, cujas dimensões e características estão apresentadas no capítulo 3.2 (tabela 3.1), foram obtidas experimentalmente as curvas de corrente de dreno em função da tensão aplicada à porta (IDS $\times$ VGS) com tensão de dreno ( $V_{D S}$ ) de $0,9 \mathrm{~V}$ e 1,2 $\mathrm{V}$ e temperatura variando de $300 \mathrm{~K}$ à $420 \mathrm{~K}$. Os resultados obtidos para os NW-TFET com dois diâmetros diferentes, $98 \mathrm{~nm}$ e $213 \mathrm{~nm}$, com 20 nanofios em paralelo (NWs) e VDS de 0,9 estão plotados na figura 3.7 .

A caracterização elétrica em função da temperatura é utilizada para melhor entender os mecanismos de condução, já que o BTBT é pouco sensível à temperatura, enquanto o SRH e o TAT são muito sensíveis.

Figura 3.7 - Curvas experimentais de IDs $x V_{G S}$ para dispositivos com fonte de $\mathrm{Si}_{0,73} \mathrm{Ge}_{0,27} \mathrm{e}$ diferentes diâmetros em função da temperatura

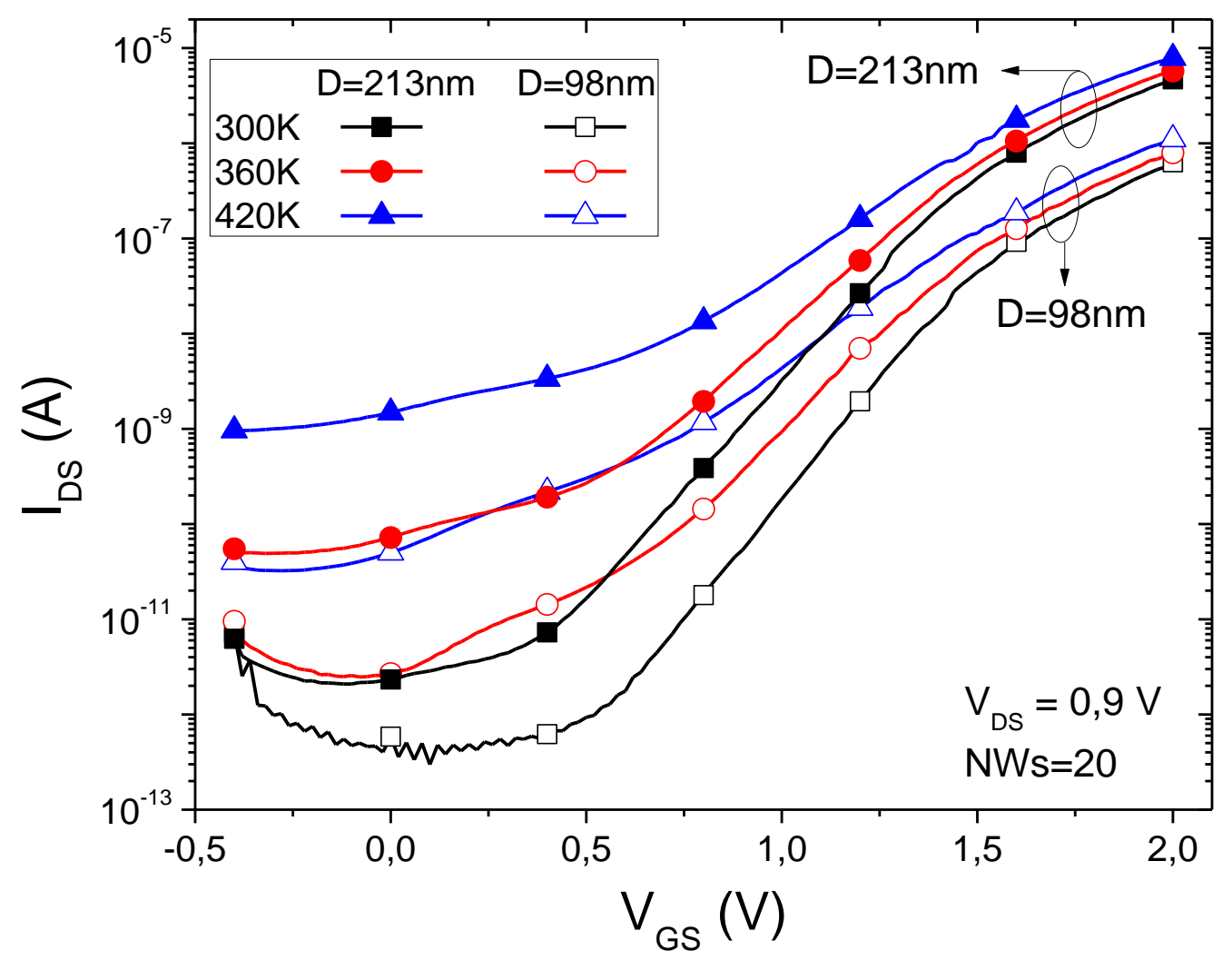

Fonte: Autor 
Observando figura 3.7, nota-se o aumento da corrente de dreno devido ao aumento do diâmetro do NW-TFET. Além disso, verifica-se que devido à elevação da temperatura, a corrente de dreno aumenta para todos os valores de tensão de porta, sendo este aumento relativamente menor para alta tensão de porta e relativamente maior para baixa tensão de porta. Conforme explicado, o mecanismo de condução predominante na corrente de desligamento do dispositivo (OFF State) deve-se principalmente à recombinação e geração $\mathrm{SRH}$, enquanto a corrente para o dispositivo em estado ligado (ON State) depende do tunelamento assistido por armadilhas (TAT) e do tunelamento de banda para banda (BTBT), a influência de cada um dos mecanismos de condução na corrente de dreno em função da tensão aplicada a porta está ilustrada na figura 3.8.

Figura 3.8 - Curva experimental de IDS $x V_{G S}$ para um TFET com fonte de $S_{0,73} G_{0,27}$, apresentando a influência predominante dos mecanismos de condução em cada região de polarização

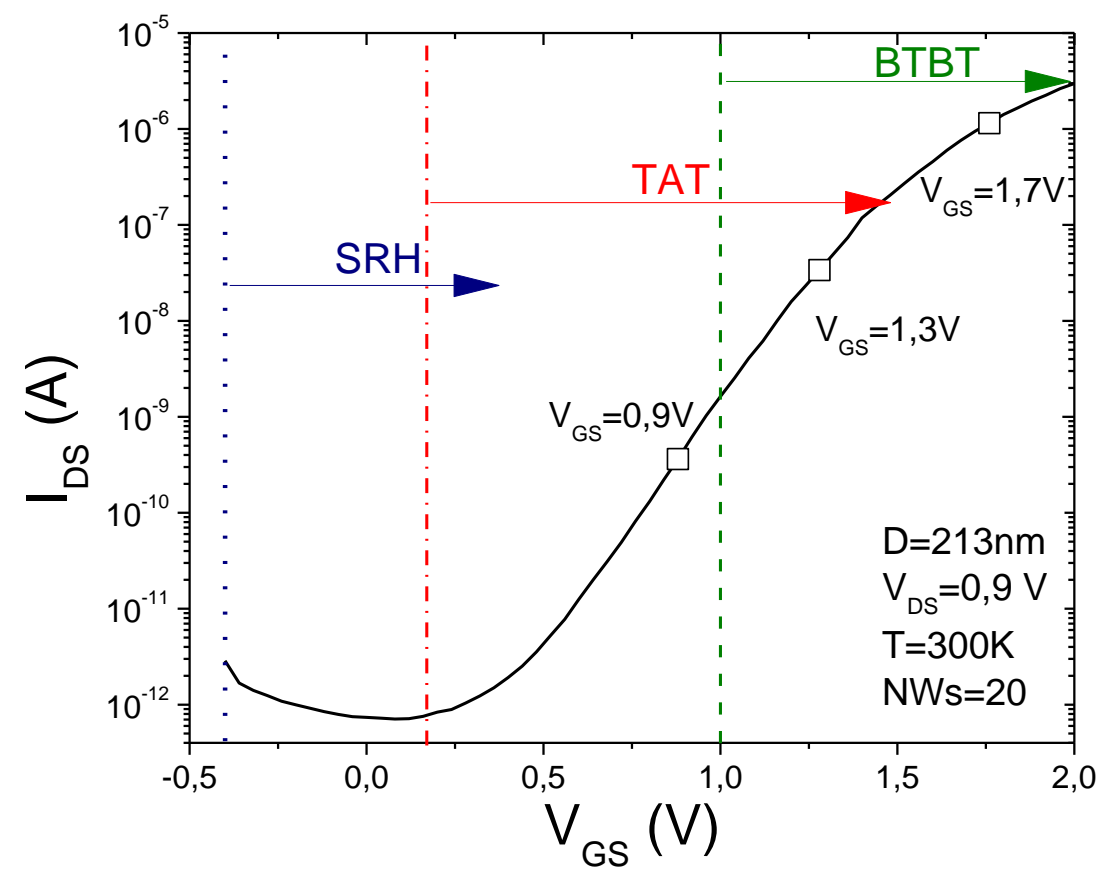

Fonte: Autor

Cada um dos mecanismos de condução apresentam uma dependência especifica com tensão e temperatura conforme apresentado no capítulo 2.2.3. A energia de ativação $\left(E_{A}\right)$ dependente do campo elétrico pode ser calculada através do método de Arrhenius (39-40), utilizando medidas experimentais em função da 
temperatura. Os mecanismos termicamente assistidos, como geração e recombinação SRH e TAT, tem uma forte sensibilidade à temperatura, apresentando altos valores de $E_{A}$, em torno de $E_{g} / 2$ e $E_{g} / 2+\Delta E_{T}$, respectivamente (41), entretanto, o BTBT apresenta valores tipicamente menores que $0,1 \mathrm{eV}$. Os resultados obtidos estão apresentados na figura 3.9, onde nota-se que o BTBT é predominante para valores de $\mathrm{V}_{\mathrm{GS}}$ acima de $1,5 \mathrm{~V}$, estando de acordo com o que foi previamente apresentado.

Figura 3.9 - Curva de $\mathrm{E}_{\mathrm{A}} \times \mathrm{V}_{\mathrm{GS}}$ para um NW-TFET com fonte de $\mathrm{Si}_{0,73} \mathrm{Ge}_{0,27}$

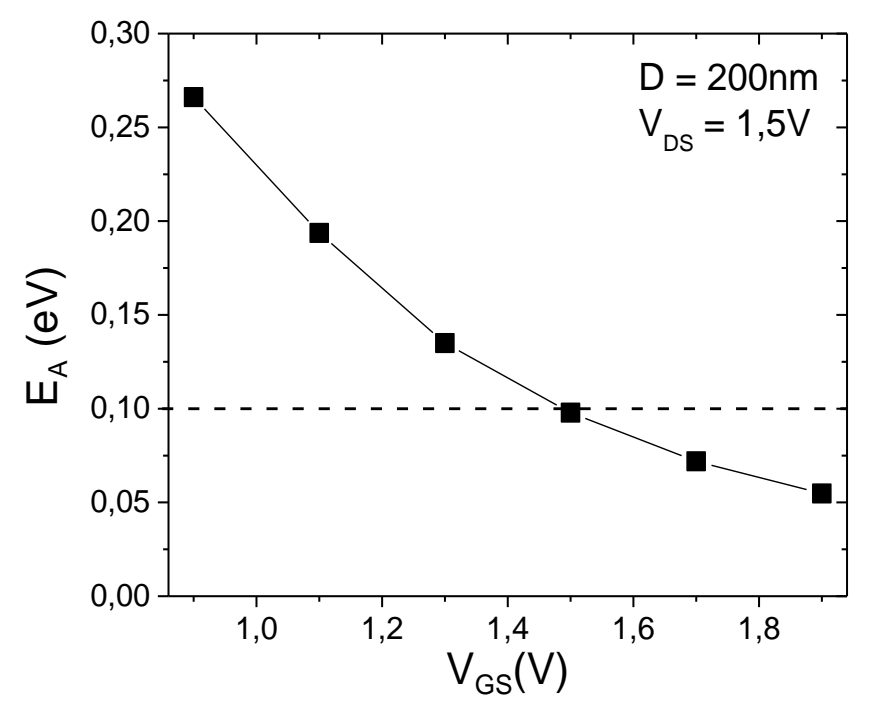

Fonte: Autor

Analisando a tendência da inclinação de sublimiar das curvas apresentadas na figura 3.7, nota-se que com o aumento da temperatura, a inclinação de sublimiar tornase pior. Esta característica pode ser justificada pelo aumento relativo da corrente de desligamento (lofF) devido ao aumento da recombinação $\mathrm{SRH}$ e da corrente de fuga térmica reversa, enquanto a corrente (ION) do dispositivo ativo apresenta uma pequena variação.

Para analisar estes dados foram definidas como corrente lon e loff a corrente de dreno (IDS) para tensão de porta (VGS) de $2 \mathrm{~V}$ e $0 \mathrm{~V}$, respectivamente.

A figura 3.10 apresenta a relação lon/loff em função da temperatura para dois diâmetros diferentes. Analisando os dois dispositivos, nota-se que ambos apresentam o mesmo comportamento com a variação da temperatura. O aumento da temperatura 
causa uma degradação na relação lon/loff, fazendo com o que os dispositivos TFET tornem-se menos interessantes para aplicações digitais em altas temperaturas.

Figura 3.10 - Relação lon/loff x T para NW-TFET com fonte de $\mathrm{Si}_{0,73} \mathrm{Ge}_{0,27}$ e dois diâmetros diferentes

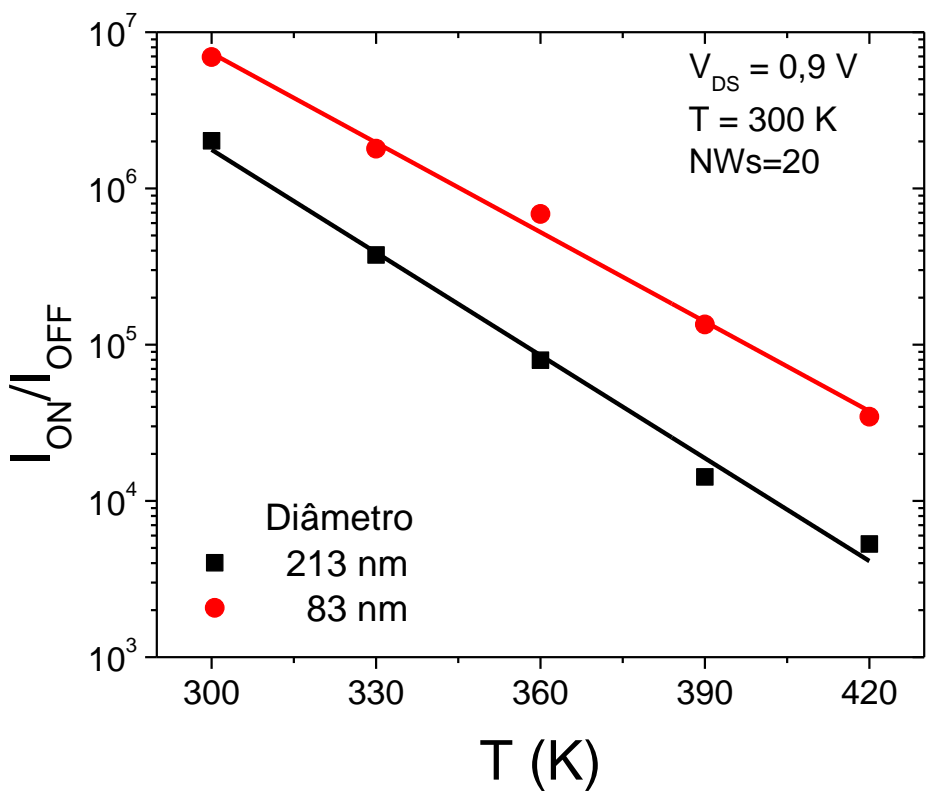

Fonte: Autor

Foram obtidas experimentalmente as curvas de corrente de dreno em função da tensão aplicada ao dreno (IDS $\times V_{D S}$ ) com $V_{G S}$ de $0,9 \mathrm{~V}, 1,3$, e 1,7 V e temperatura variando de $300 \mathrm{~K}$ a $420 \mathrm{~K}$, para um NW-TFET de $213 \mathrm{~nm}$ de diâmetro. Os resultados estão demonstrados na figura 3.11.

É interessante notar que, embora o conceito inicial do TFET seja para aplicações digitais devido à sua alta velocidade de chaveamento, os dados apresentados na figura 3.11 demonstram que esse dispositivo também pode ser interessante para aplicações analógicas. Esta ideia baseia-se na região quase plana mostrado na corrente de dreno (IDS) para altas tensões de dreno (VDS), apresentando uma corrente de saturação praticamente estável para todas as tensões de porta (VGS), ilustrando a baixa susceptibilidade ao VDS.

Analisando o impacto da temperatura na região de saturação das curvas apresentadas na figura 3.11, mais uma vez observa-se que a corrente de dreno depende do principal mecanismo de condução em cada polarização. 
Figura 3.11 - Curvas experimentais de IDS $\times V_{D S}$ com diferentes valores de $V_{G S}$ e em função da temperatura para NW-TEFET com fonte de $\mathrm{Si}_{0,73} \mathrm{Ge}_{0,27}$

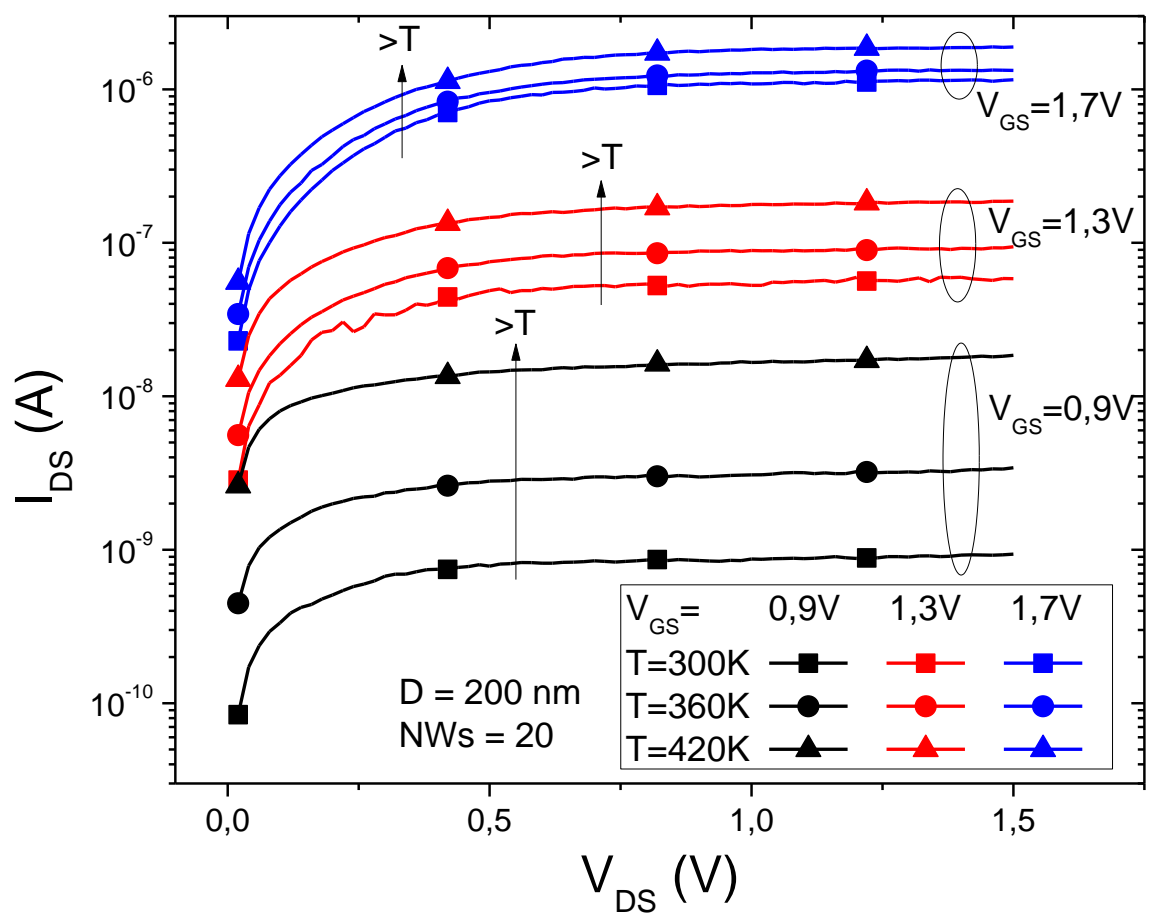

Fonte: Autor

Para tensões de porta mais altas $\left(\mathrm{V}_{\mathrm{GS}}=1,7 \mathrm{~V}\right)$, o mecanismo de condução predominante é devido ao tunelamento direto de banda para banda, resultando em uma pequena variação relativa da corrente de dreno. Para tensões intermediárias ( $V_{G S}=1,3 \mathrm{~V}$ e 0,9 V), além do BTBT, ocorre influência do tunelamento induzido por armadilhas, fazendo com que haja uma variação um pouco maior da corrente de dreno.

Comparando as curvas de IDS $x$ VDS para dispositivos com diferentes diâmetros e com tensão de porta de 1,3 V, apresentado na figura 3.12, é possível observar o que foi previamente mencionado, o aumento da corrente de dreno com o aumento do diâmetro. 
Figura 3.12 - Curvas experimentais de IDS $\times V_{\text {DS }}$ para NW-TFET com fonte de $\mathrm{Si}_{0,73} \mathrm{Ge}_{0,27}$, com diâmetros diferentes e em função da temperatura

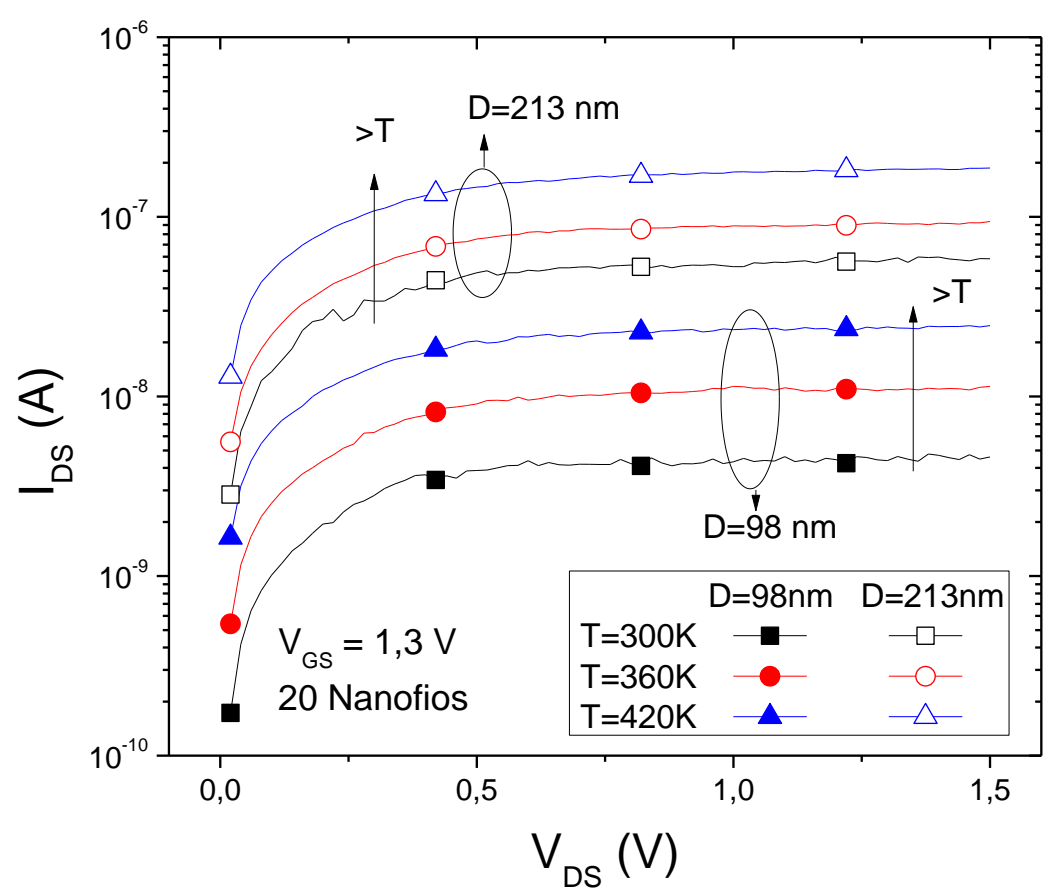

Fonte: Autor

\subsection{Influência do material de fonte no NW-TFET}

Neste capítulo será estudada a influência de diversos materiais de fonte no comportamento destes dispositivos. Serão utilizadas as lâminas 2, 3 e 4, onde as fontes são compostas por: $\mathrm{Si}_{0,73} \mathrm{Ge}_{0,27}, \mathrm{Si}_{0,54} \mathrm{Ge}_{0,46}$ e $\mathrm{Si}$, respectivamente. As dimensões de cada uma delas estão apresentadas na tabela 3.1, sendo: comprimento total de canal $\left(L_{C H}\right)$ de $160 \mathrm{~nm}$, comprimento de porta $\left(L_{G}\right)$ de $150 \mathrm{~nm}$, sobreposição de porta na região da fonte (LGS) de $30 \mathrm{~nm}$, subposição de porta na região de dreno (LGD) de $40 \mathrm{~nm}$, espessura equivalente de óxido (EOT) de $3 \mathrm{~nm}$ e diâmetro (D) de 200 $\mathrm{nm}$. Região de fonte dopada com $N_{A}=1.10^{20} \mathrm{~cm}^{-3}$, canal com $N_{D}=1.10^{15} \mathrm{~cm}^{-3}$ e dreno com $\mathrm{ND}_{\mathrm{D}}=1.10^{20} \mathrm{~cm}^{-3}$. Foram medidas cascatas com 100 nanofios (NWs) em paralelo.

Duas variações do dispositivo com fonte de Si foram utilizadas. Uma delas foi obtida através do processo de crescimento epitaxial de $\mathrm{Si}$, seguido pela dopagem por implantação iônica, conforme apresentado no capítulo 3.1, o que resulta em uma junção canal/fonte não abrupta. A outra foi obtida através do método de substituição, conforme será apresentado no capítulo 4.1, gerando uma estrutura com uma junção aproximadamente abrupta na região canal/fonte 
A figura 3.13 apresenta a curva experimental de IDS $\times$ VGs em temperatura ambiente para os quatro NW-TFETs com diferente composição de fonte. Conforme já demonstrado, a presença de $\mathrm{Si}_{1-x} \mathrm{Ge}_{\mathrm{x}}$, e consequentemente redução de $\mathrm{E}_{\mathrm{g}}$, resulta no aumento da corrente de funcionamento do dispositivo (IoN), sem aumentar a corrente de desligamento (lofF), impactando diretamente na melhora da inclinação de sublimiar (SS). Observa-se também que o NW-TFET com fonte de Si e junção abrupta apresenta maior lon quando comparado ao com junção não abrupta, indicando uma melhora nos mecanismos de tunelamento.

Figura 3.13 - Curvas experimentais de IDS $x$ VGs para NW-TFET com diferentes composições de fonte

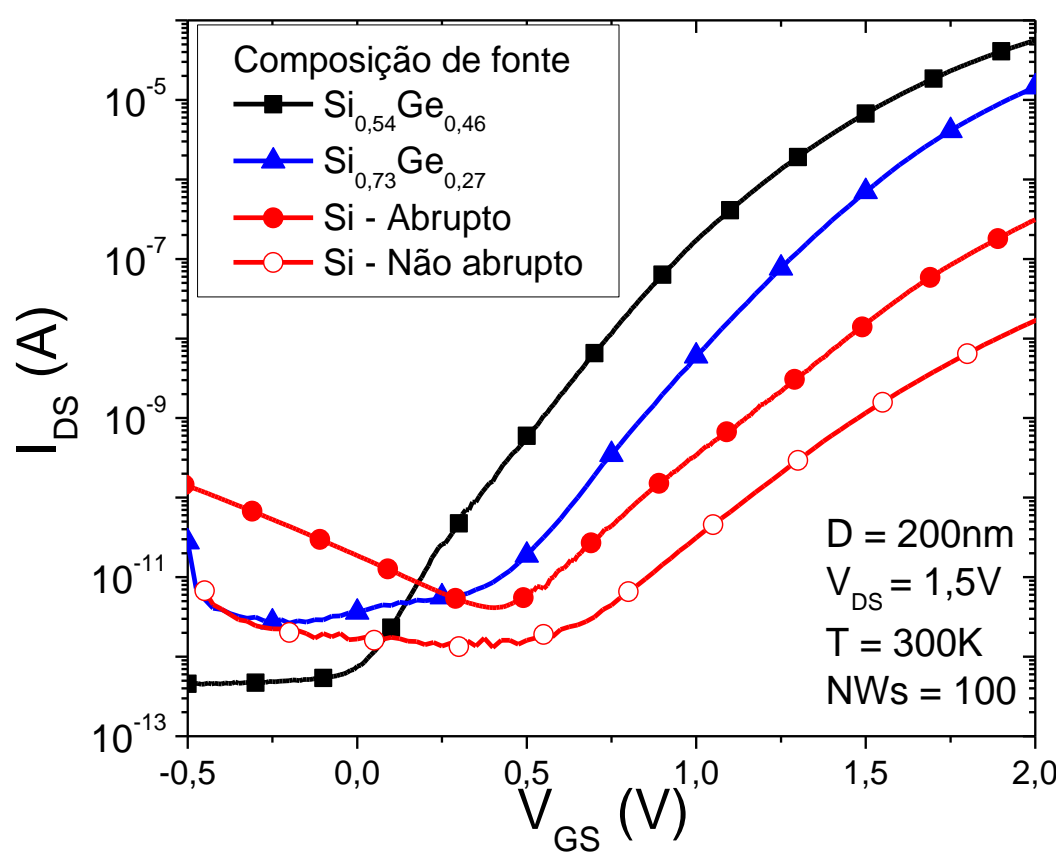

Fonte: Autor

As curvas de IDS em função de $V_{D S}$ em temperatura ambiente para os dispositivos com diferentes composições de fonte estão plotadas na figura 3.14. Conforme já demonstrado, para altas tensões de $V_{D S}$, O NW-TFET apresenta uma corrente de saturação praticamente estável, se assemelhando com a região de saturação de um transistor MOSFET. E assim como nas curvas de IDS $\times V_{G S}$, o dispositivo com maior concentração de Ge apresenta níveis maiores de IDS, devido ao menor $E_{g}$ e consequentemente maior taxa de tunelamento. 
Figura 3.14 - Curvas experimentais de IDS $\times$ VDS para NW-TFET com diferentes composições de fonte

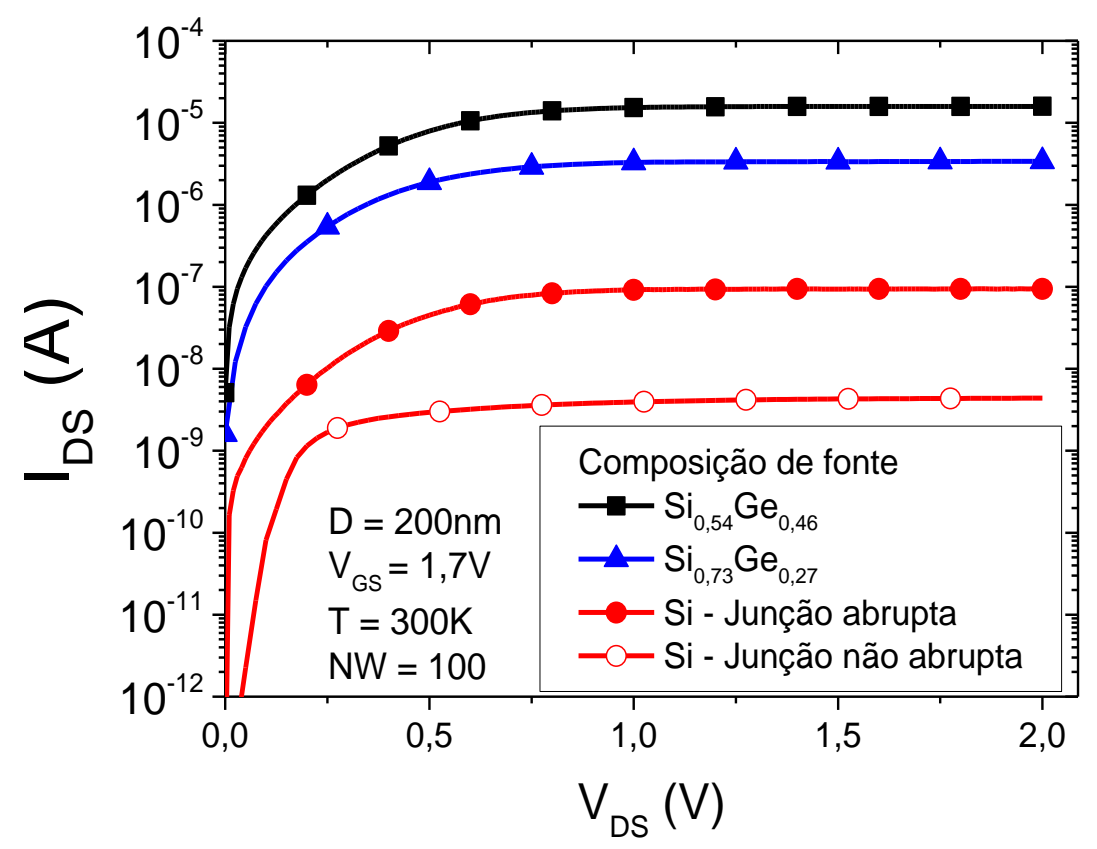

Fonte: Autor

A influência de altas temperaturas no comportamento destes transistores está demonstrada na figura 3.15 e 3.16. Estas figuras apresentam as curvas de corrente de dreno (IDS) em função da tensão aplicada a porta ( $V_{G S)}$ com temperatura variando de $300 \mathrm{~K}$ à $425 \mathrm{~K}$ para os dispositivos com fonte de $\mathrm{Si}_{1-\mathrm{x}} \mathrm{Ge}_{\mathrm{x}}$ e Si.

Conforme apresentado no capítulo 2.3.4, o aumento da temperatura resulta no aumento da corrente de tunelamento induzido por armadilhas (TAT) devido a sua dependência direta com a temperatura, enquanto o BTBT apresenta uma dependência indireta através da variação de $E_{g}$ com a temperatura.

A partir das curvas de lDS $x V_{G s}$ plotadas é possível observar a menor influência da temperatura na região de lon nos dispositivos com fonte de $\mathrm{Si}_{1-x} \mathrm{Ge}_{\mathrm{x}}$ (figura 3.15), quando comparado aos com fonte de Si (figura 3.16). 
Figura 3.15 - Curvas experimentais de IDS $x V_{G S}$ em função da Temperatura para NW-TFET com fonte de $\mathrm{Si}_{1-x} \mathrm{Ge}_{x}$

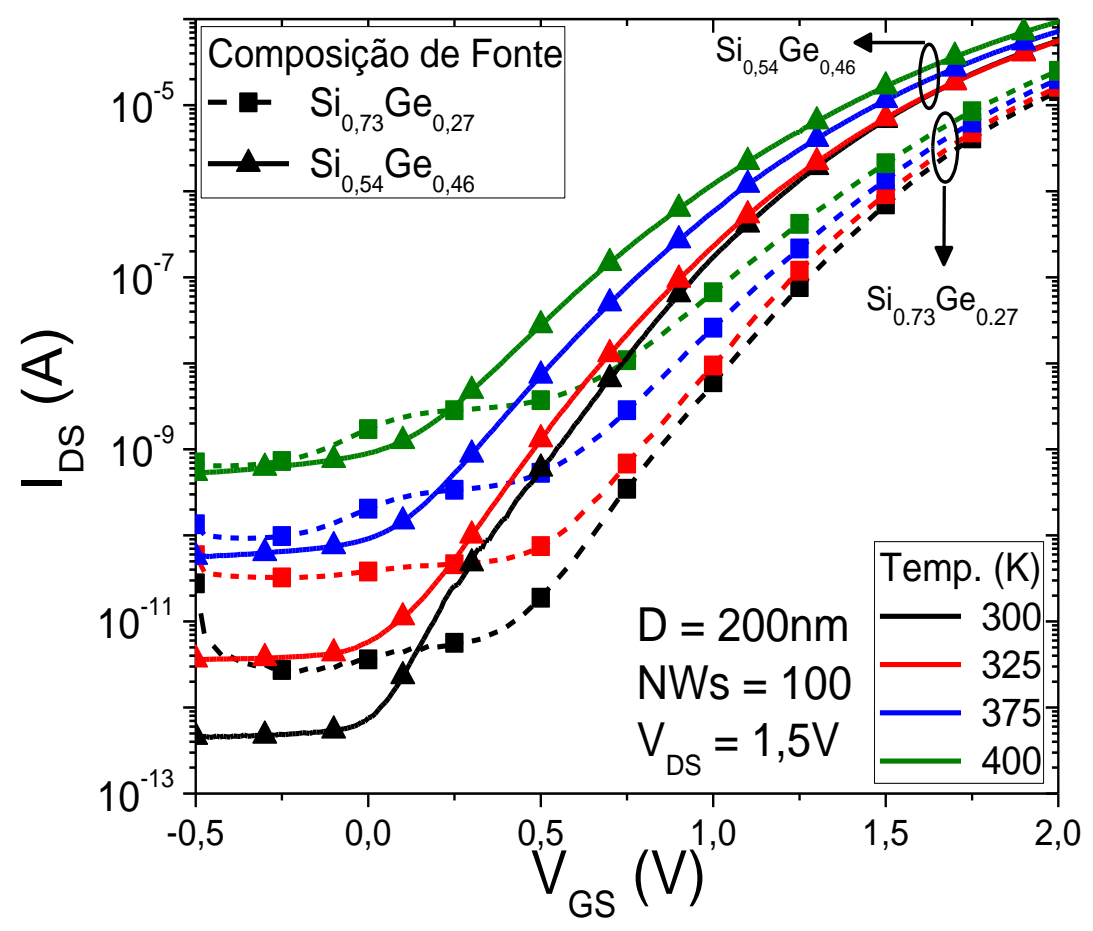

Fonte: Autor

Figura 3.16 - Curvas experimentais de lDS $x V_{G S}$ em função da Temperatura para NW-TFET com fonte de $\mathrm{Si}$

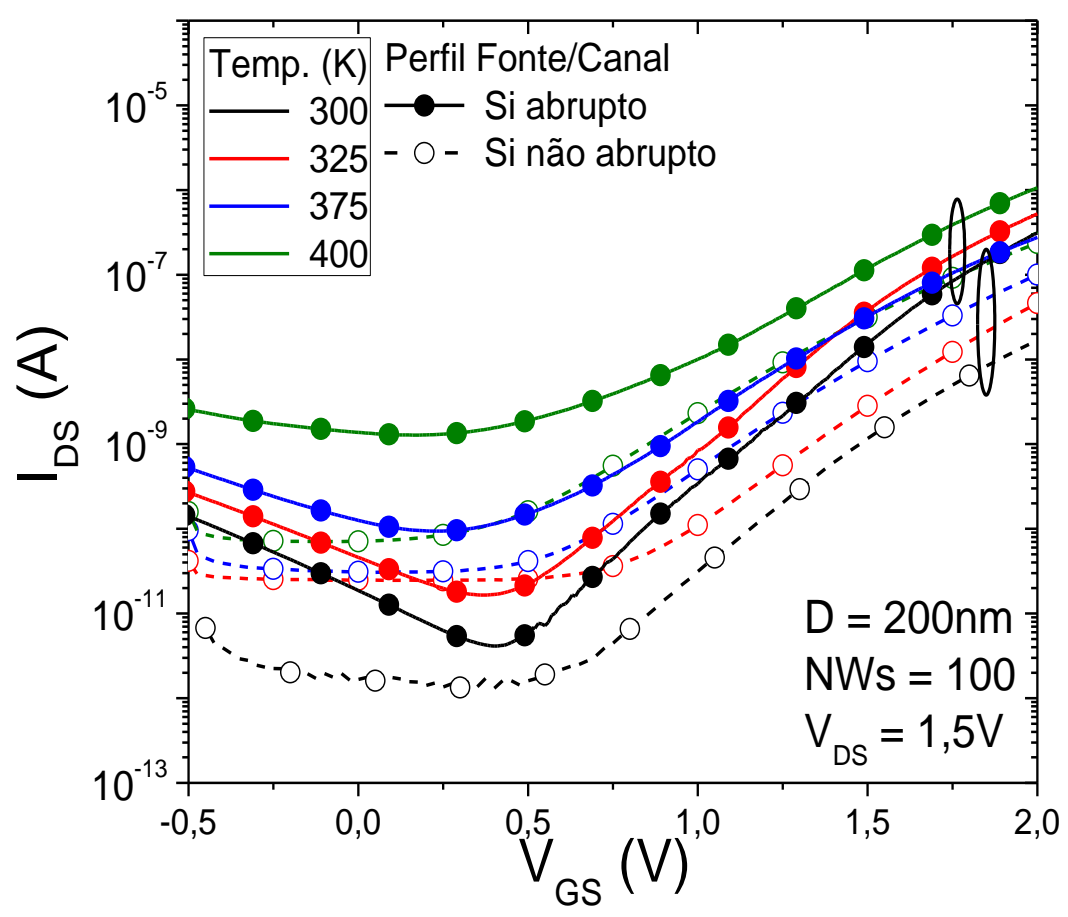

Fonte: Autor 
Para melhor analisar essa influência da temperatura e atuação de cada um dos mecanismos de condução, foram extraídas as curvas de energia de ativação equivalente $\left(E_{A}\right)$ em função de $V_{G S}$ através do método de Arrhenius (39-40) (figura 3.17). Considerando que os mecanismos termicamente assistidos, como TAT e geração e recombinação $S R H$ apresentam valores mais altos de $E_{A}$, enquanto $B T B T$ apresenta valores tipicamente menores do que $0,1 \mathrm{eV}$, observa-se que os transistores NW-TFET com fonte de $\mathrm{Si}_{1-\mathrm{x}} \mathrm{Ge}_{\mathrm{x}}$ são dominados por BTBT para $\mathrm{V}_{\mathrm{GS}}>1,5 \mathrm{~V}$.

Porém, ambos os dispositivos com fonte de $\mathrm{Si}$ apresentam $\mathrm{E}_{\mathrm{A}}>0,1$ para $V_{G S}>2 \mathrm{~V}$, indicando que não atingiram BTBT e são dominados predominantemente por TAT, justificando a maior dependência com a temperatura na região de loN. Entretanto, vale ressaltar que o dispositivo com junção abrupta está próximo de atingir BTBT em $V_{G S}=2 \mathrm{~V}$, justificando os níveis de IDs mais altos quando comparado ao não abrupto.

Figura 3.17 - Curvas experimentais de $E_{A} \times V_{G S}$ para NW-TFET com diferentes composições de fonte

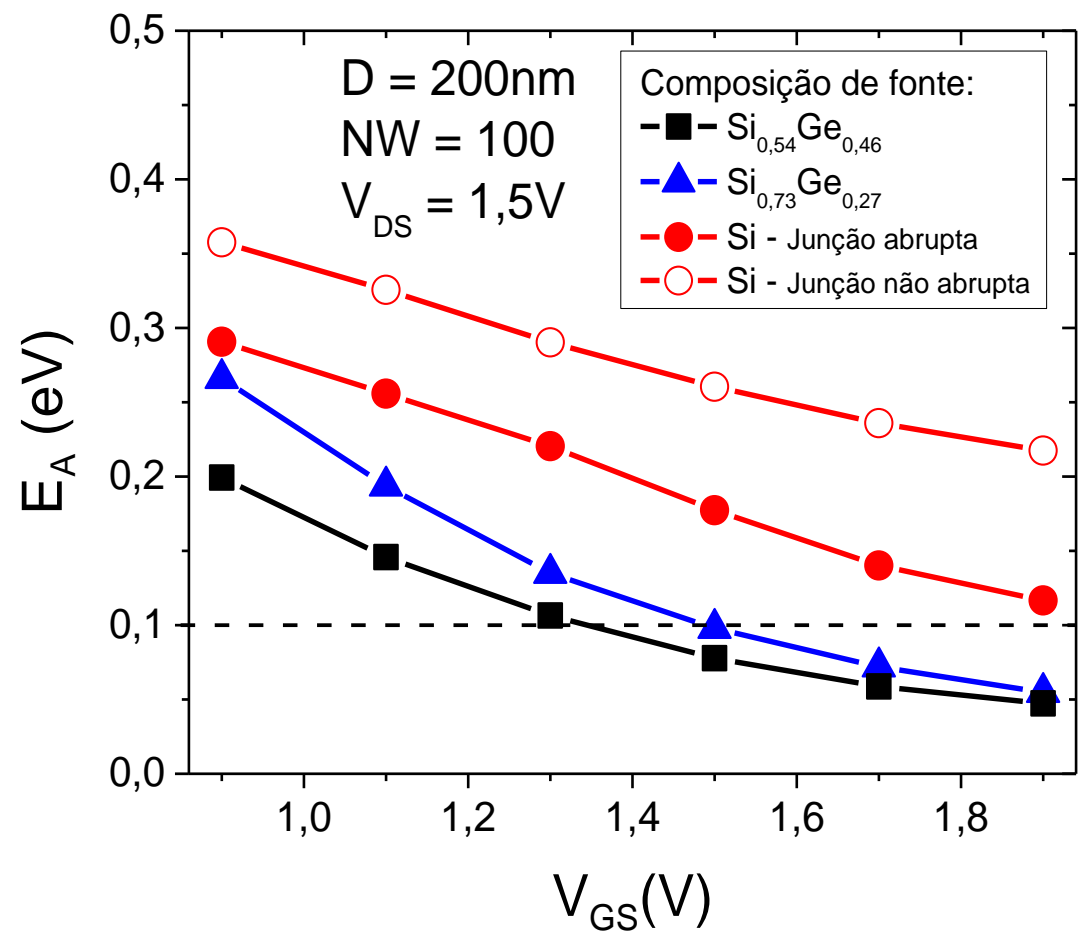

Fonte: Autor 


\subsection{Parâmetros analógicos do NW-TFET com fonte de $\mathrm{Si}_{1-\mathrm{x}} \mathrm{Ge}_{\mathrm{x}}$ em função da temperatura}

Apesar do TFET ter sido desenvolvido com o intuito de aplicações digitais devido ao seu chaveamento mais rápido ( $S S<60 \mathrm{mV} / \mathrm{dec}$ ), o processo de fabricação dos NW-TFET ainda não se encontra otimizado, fazendo com que este apresente inclinação de sublimiar superior a $100 \mathrm{mV} / \mathrm{dec}(25,26)$. Porém, conforme apresentado no capítulo anterior e na referência (51), esses dispositivos têm apresentados alguns resultados analógicos promissores.

Uma figura de mérito importante para caracterizar o comportamento analógico de um transistor é ganho intrínseco de tensão $(A v)$, dado pela relação entre transcondutância $\left(\mathrm{g}_{\mathrm{m}}\right)$ e a condutância de saída $\left(\mathrm{g}_{\mathrm{d}}\right)$ conforme apresentado na equação 3.1 .

$$
\left|A_{V}\right|=\frac{g_{m}}{g_{d}}
$$

O $g_{m}$ mede a eficácia do controle de corrente de dreno pela tensão de porta e é dada pela equação 3.2, enquanto o gd é dado pela variação da corrente de dreno em função da tensão aplicada ao dreno (equação 3.3), quanto menor o seu valor, melhor a sua relação.

$$
\begin{aligned}
& g_{m}=\frac{d I_{D S}}{d V_{G S}}, \text { com } V_{D S} \text { constante } \\
& g_{d}=\frac{d I_{D S}}{d V_{D S}}, \text { com } V_{G S} \text { constante }
\end{aligned}
$$

A figura 3.18 apresenta as curvas de $\mathrm{g}_{\mathrm{m}}$ extraídos para $\mathrm{V}_{\mathrm{GS}}=1,7 \mathrm{~V} \mathrm{e}$ $\mathrm{V}_{\mathrm{DS}}=1,5 \mathrm{~V}$ e temperatura variando de $300 \mathrm{~K}$ à $425 \mathrm{~K}$. Pode-se observar que os dispositivos com maior concentração de Ge na fonte apresentam maior nível de $\mathrm{gm}_{\mathrm{m}}$, seguidos pelo dispositivo com fonte de $\mathrm{Si}$ e junção abrupta. Além disso, nota-se que para os transistores com fonte de $\mathrm{Si}_{1-x} \mathrm{Ge}_{\mathrm{x}}$, devido a predominância de BTBT, para esta condição de polarização quase não há influência da variação de temperatura. 
O $g_{m}$ dos dispositivos com fonte de Si e junção abrupta, apresentam um comportamento similar aos com fonte de $\mathrm{Si}_{1-x} \mathrm{Ge}_{\mathrm{x}}$. Nesta condição de polarização, apesar do mecanismo de condução predominante ser o TAT, existe uma parcela considerável de BTBT, como pode ser inferido na figura 3.17, $\mathrm{E}_{\mathrm{A}}=0,14$ para $\mathrm{V}_{\mathrm{GS}}=1,7 \mathrm{~V}$. Entretanto, o dispositivo com fonte de Si e junção não abrupta apresenta maior variação relativa de $g_{m}$, sendo justificado pela predominância de TAT com influência mínima de $B T B T$, como pode ser observado na figura $3.17, E_{A}=0,23$ para $V_{G S}=1,7 \mathrm{~V}$.

A partir da equação 2.7 é possível notar que o BTBT é fortemente dependente do campo elétrico, sendo assim para tensões mais altas de $V_{D S}$, maior o campo elétrico, levando à maior corrente de tunelamento e transcondutância. Em contrapartida, ocorre o aumento da condutância de saída $\left(g_{d}\right)$, o que significa maior degradação de gd, como pode ser visto na figura 3.19.

Figura 3.18 - Curvas experimentais de $g_{m} \times$ T para NW-TFET com diferentes composições de fonte

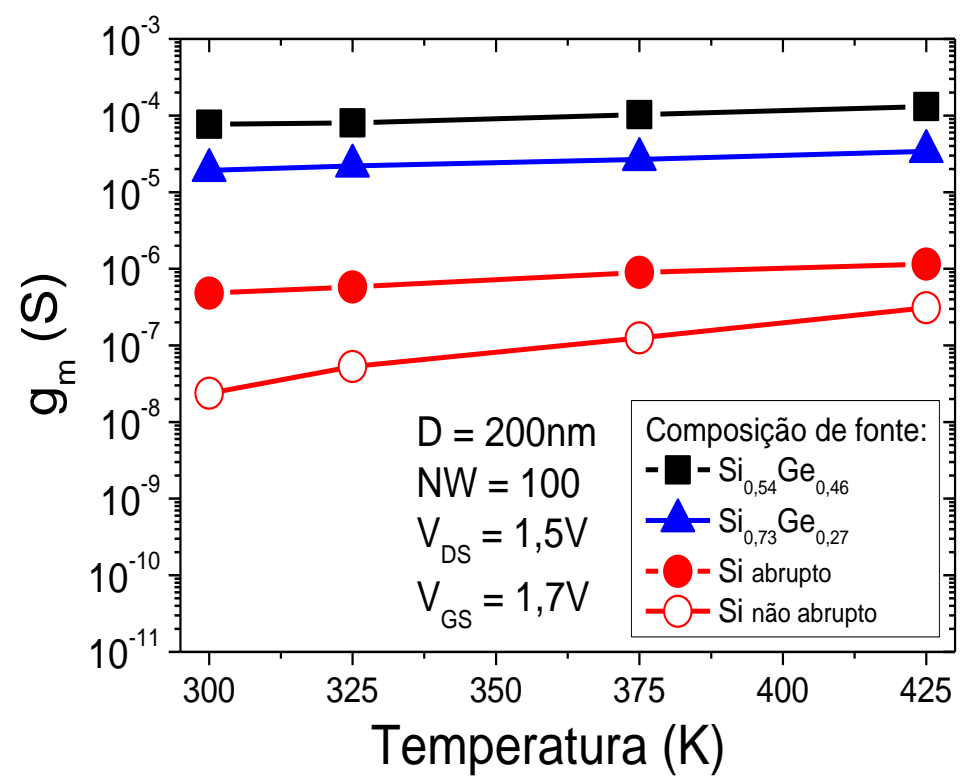

Fonte: Autor 
Figura 3.19 - Curvas experimentais de $g_{d} \mathrm{X}$ T para NW-TFET com diferentes composições de fonte

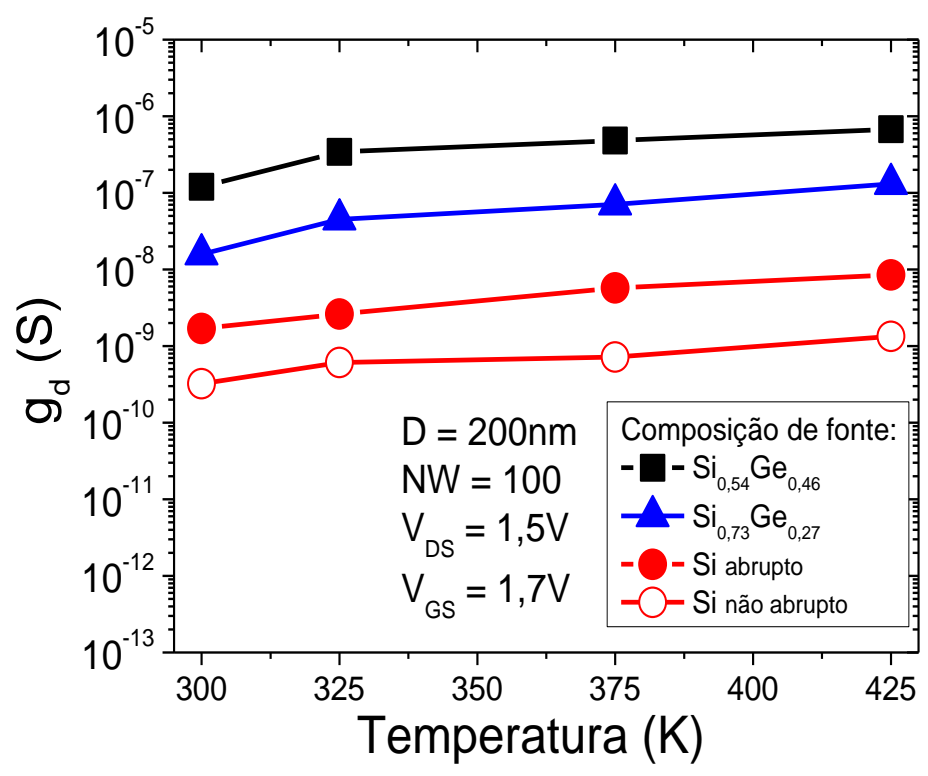

Fonte: Autor

Ao analisar a influência da temperatura no $g_{d}$, nota-se que todos os dispositivos apresentam uma variação relativa muito similar com a variação de temperatura.

A partir da equação 3.1 foram obtidos os valores Av para todos os dispositivos estudados neste capítulo, os valores em função da temperatura estão plotados na figura 3.20. A partir deste gráfico, observa-se que é possível obter diferentes tendências de Av dependendo dos mecanismos de condução predominantes.

Figura 3.20 - Curvas experimentais de Av x T para NW-TFET com diferentes composições de fonte.

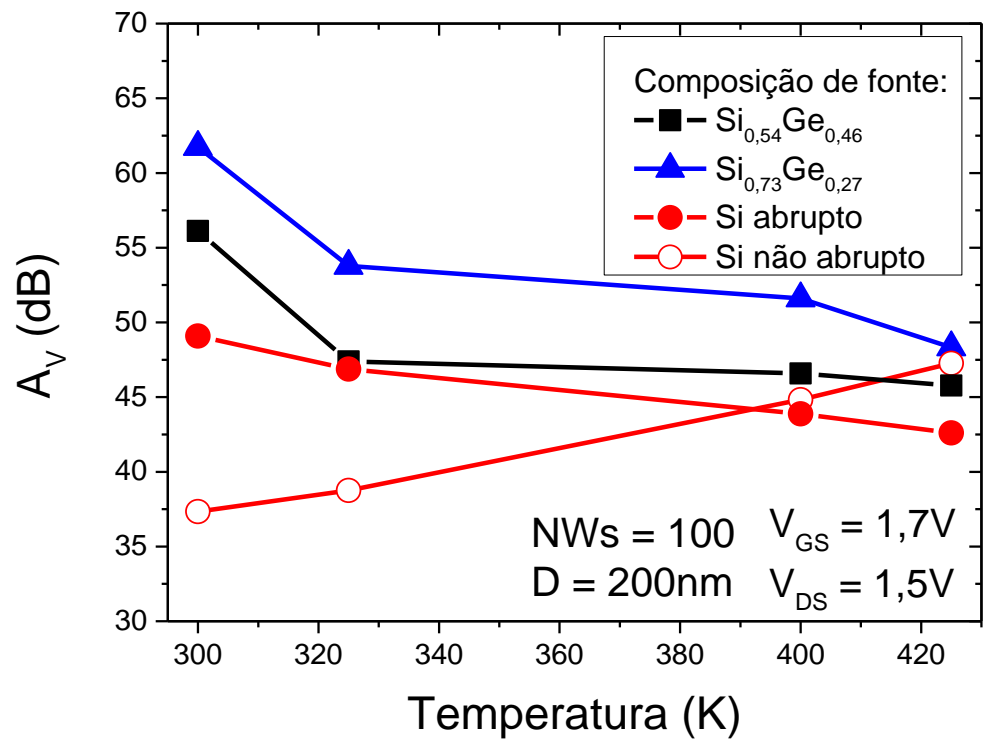

Fonte: Autor. 
Os transistores com fonte de $\mathrm{Si}_{1-x} \mathrm{Ge}_{\mathrm{x}}$, dominados por BTBT apresentam uma forte diminuição de Av com o aumento de temperatura, o que pode ser claramente justificado pela maior variação de $g_{d}$ quando comparado à variação de $g_{m}$ com a temperatura. Além disso, o NW-TFET com maior concentração de Ge $(x=0,46)$ apresenta piores resultados quando comparado ao com menor concentração de Ge $(x=0,27)$, graças a maior degradação da condutância de saída $\left(g_{d}\right)$.

Os dispositivos com fonte de Si e junção abrupta, por estar próximo à predominância de BTBT em tensões de polarização mais altas, apresentaram a mesma tendência quando comparado aos com fonte de $\mathrm{Si}_{1-x} \mathrm{Ge}$. Porém, nota-se que os dispositivos com fonte de Si e junção não abruptas, apresentaram um comportamento totalmente contrário, sendo explicado pelo maior aumento de $\mathrm{gm}_{\mathrm{m}}$ conforme observado na figura 3.18, sendo assim, demostrando um pior desempenho analógico para temperaturas mais baixas, mas uma melhora neste comportamento para altas temperaturas, apresentando valores de Av superiores ao dispositivo com fonte de $\mathrm{Si}_{0,54} \mathrm{Ge}_{0,46} \mathrm{em} \mathrm{T}=420 \mathrm{~K}$.

\subsection{Influência da densidade de armadilhas de interface para NW-TFET com diferentes materiais de fonte}

Nesta etapa do trabalho foram obtidas medidas experimentais a partir das lâminas 2 e 4, apresentadas no capítulo 3.2, cujas dimensões são: comprimento total de canal $\left(L_{C H}\right)$ de $160 \mathrm{~nm}$, comprimento de porta $\left(L_{G}\right)$ de $150 \mathrm{~nm}$, sobreposição de porta na região da fonte (LGs) de $30 \mathrm{~nm}$, subposição de porta na região de dreno (LGD) de $40 \mathrm{~nm}$, espessura equivalente de óxido (EOT) de $3 \mathrm{~nm}$ e diâmetro (D) de $200 \mathrm{~nm}$. Região de fonte dopada com $N_{A}=1.10^{20} \mathrm{~cm}^{-3}$, canal com $N_{D}=1.10^{15} \mathrm{~cm}^{-3}$ e dreno com $N_{D}=1.10^{20} \mathrm{~cm}^{-3}$. Fonte projetada com liga $\mathrm{Si}_{0,73} \mathrm{Ge}_{0,27}$ e Si. Foram medidas cascatas com 100 nanofios em paralelo.

A figura 3.21 apresenta curvas de corrente de dreno em função da tensão aplicada à porta, obtidas experimentalmente, para dois NW-TFET com fontes de Si e liga $\mathrm{Si}_{0,73} \mathrm{Ge}_{0,27}$, com tensão de dreno de $1,5 \mathrm{~V}$ e em temperatura ambiente.

É possível observar que devido à redução da banda proibida $\left(E_{g}\right)$ do material de fonte, houve um aumento de duas décadas na corrente de dreno na região lon, 
sendo isso devido ao aumento da corrente de tunelamento (BTBT). Além disso, na região de loff, também é possível observar que o uso de liga SiGe promove o aparecimento de um "platô" na curva, entre 0,2 V e 0,5 V.

Considerando que o aumento da quantidade de germânio na fonte, pode causar um aumento na densidade de defeitos na interface da junção fonte/canal ( $\mathrm{N}_{\text {it }}$ ). $A$ corrente de dreno torna-se mais dependente do tunelamento induzido por armadilhas (TAT), aumentando IDs para baixos valores de tensão de porta, onde este passa a ser o mecanismo de transporte predominante.

Figura 3.21 - Curvas experimentais de IDS $\times V_{G S}$, comparando NW-TFET com fontes de diferentes materiais

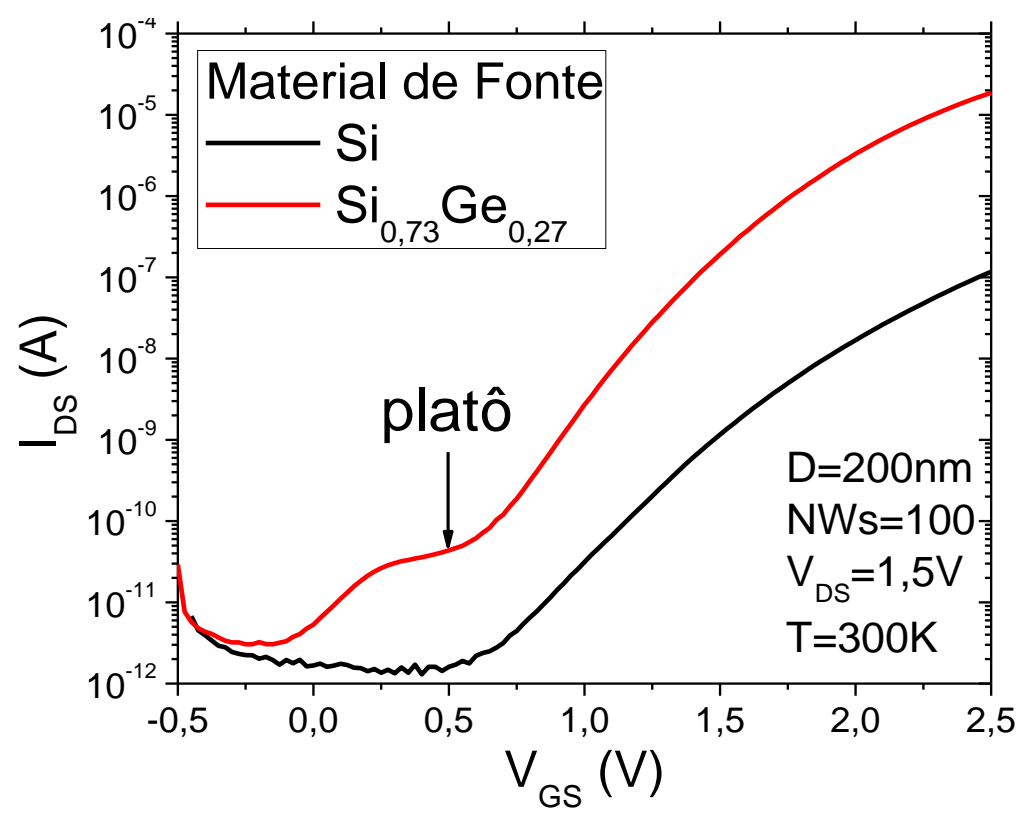

Fonte: Autor

Para melhor entender como cada mecanismo de transporte é influenciado pelas armadilhas de interface ( $\mathrm{Nit}_{\mathrm{it}}$ ) e como isso influencia no comportamento do NW-TFET, foram realizadas simulações numéricas bidimensionais de dispositivos com características e dimensões similares aos fabricados no Imec/Bélgica e descritos acima. Devido à incompatibilidade dos modelos de armadilhas e construção de estruturas com heterojunções no TCAD Sentaurus, foi necessária a utilização do TCAD Silvaco, que por sua vez só possui modelos bidimensionais de tunelamento. 
A densidade de armadilhas de interface $\left(\mathrm{N}_{\mathrm{it}}\right)$ foi considerada em toda extensão da interface entre silício e oxido de porta do dispositivo, conforme demonstrado na figura 3.22 .

A figura 3.23 apresenta o comportamento do NW-TFET para diferentes densidades de armadilhas de interface $\left(\mathrm{N}_{\mathrm{it}}\right)$, obtidas a partir destas simulações.

Figura 3.22 - Figura esquemática do dispositivo NW-TFET simulado, demonstrando onde foram considerados o $\mathrm{N}_{\mathrm{it}}$

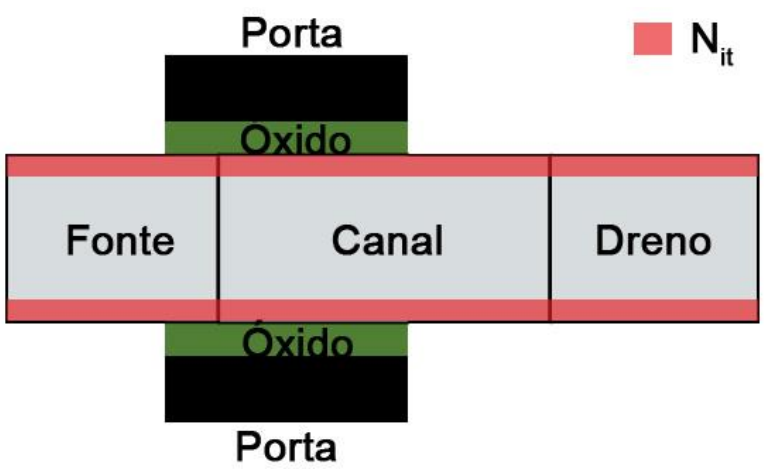

Fonte: autor

Figura 3.23 - Curvas IDS $\times V_{G S}$ variando a quantidade de $N_{i T}$ na interface fonte/canal obtida por simulações no TCAD Silvaco

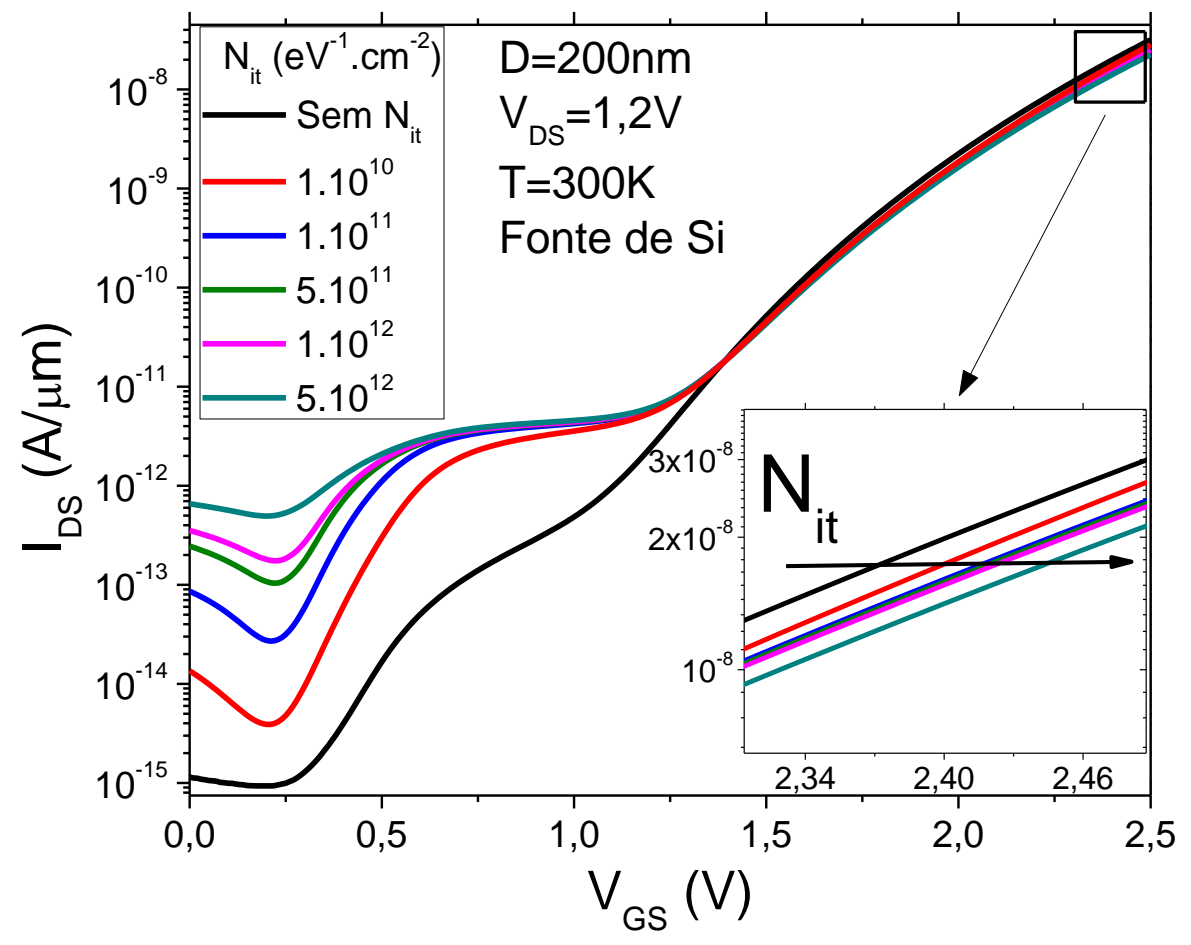

Fonte: Autor 
Para V Gs menor que 1,2 V, nota-se que o aumento das armadilhas de interface ( $\left(\mathrm{Nit}_{t}\right.$ ), ocasiona uma elevação na corrente de dreno (IDS), sendo este resultado devido ao aumento do tunelamento induzido por armadilhas (TAT). Para $\mathrm{V}_{\text {GS }}$ maior que 1,2V, observa-se que o ponto onde o tunelamento de banda para banda (BTBT) começa a prevalecer sobre os outros mecanismos de condução é atrasado devido a presença do $\mathrm{N}_{\mathrm{it}}$, estendendo a predominância de TAT para valores mais altos de $\mathrm{V}_{\mathrm{GS}}$.

Continuando com a ajuda do simulador, foram obtidas as curvas IDS $\times$ VGS considerando apenas o tunelamento de banda para banda ou somente o tunelamento induzido por armadilhas e por fim, a interação entre estas duas componentes.

Para que fosse possível realizar as simulações considerando apenas o tunelamento assistido por armadilhas, foi necessário reduzir o parâmetro de ajuste da taxa de geração de tunelamento de banda para banda $\left(A_{B T B T}\right)$ na equação 2.7 de $3,5.10^{20} \mathrm{~cm}^{-1} \mathrm{~V}^{-2} \mathrm{~s}^{-1}$ para $1.10^{15} \mathrm{~cm}^{-1} \mathrm{~V}^{-2} \mathrm{~s}^{-1}$, fazendo com que a sua influência fosse menor que a influência do modelo de tunelamento assistido por armadilhas. Em todas as curvas foram considerados baixos $\left(\mathrm{N}_{\mathrm{it}} \approx 0\right)$ e altos valores de densidade de armadilhas de interface $\left(\mathrm{Nit}_{\mathrm{it}}=1.10^{12} \mathrm{eV}^{-1} \cdot \mathrm{cm}^{-2}\right)$, conforme ilustrado na figura 3.24 .

Figura 3.24 - Curvas comparando a influência de cada mecanismo de transporte de portadores no comportamento de lDS com a variação de $V_{G S}$

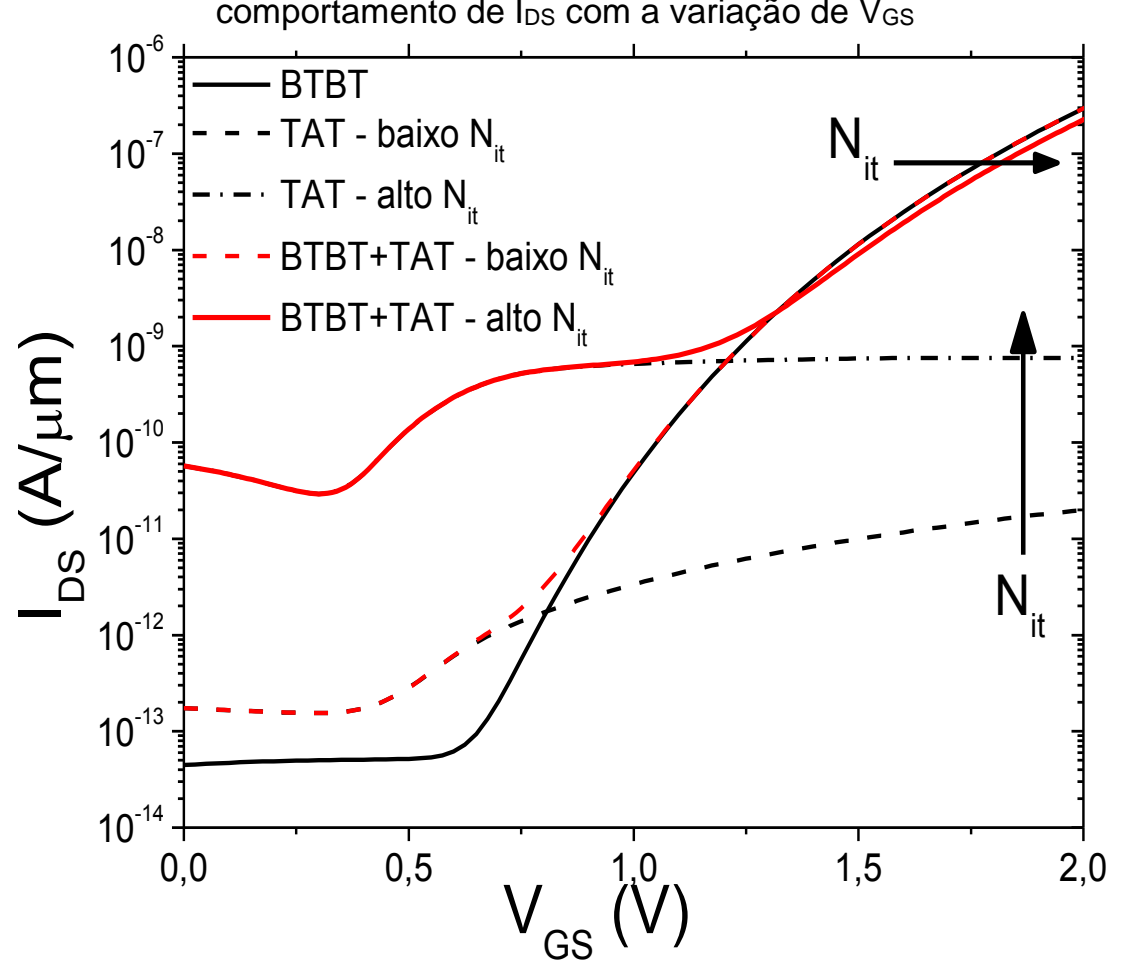

Fonte: Autor 
Para baixos valores de tensão de porta a taxa de tunelamento induzido por armadilhas é maior do que o tunelamento de banda para banda e esta diferença é ainda maior quando considerado altos valores de densidade de armadilhas de interface. Observando a interação entre as duas componentes de tunelamento (BTBT + TAT), nota-se que ocorre uma alta degradação relativa na corrente loff e menor degradação relativa da corrente lon, demonstrando a maior influência das armadilhas onde ocorre o TAT, fazendo com que aconteça um atraso do ponto onde o tunelamento de banda para banda prevalece sobre os outros mecanismos de transporte.

Para estudar a influência do germânio na região fonte, foram obtidas através de simulações curvas de IDS $x V_{\text {GS }}$ com diferentes larguras de banda proibida na região de fonte, com e sem a presença de densidades de armadilhas de interface $\left(\mathrm{N}_{\mathrm{it}}\right)$. Os resultados obtidos foram plotados na figura 3.25, onde $E_{g}=1,08 \mathrm{eV}, 0,93 \mathrm{eV} e$ $0,82 \mathrm{eV}$, equivale à $\mathrm{Si}, \mathrm{Si}_{0,73} \mathrm{Ge}_{0,27}$ e $\mathrm{Si}_{0,56} \mathrm{Ge}_{0,44}$, respectivamente.

Figura 3.25 - Curvas de lDS $x V_{G S}$ variando o $E_{g}$ do material de fonte e considerando a influência de $N_{i t}$

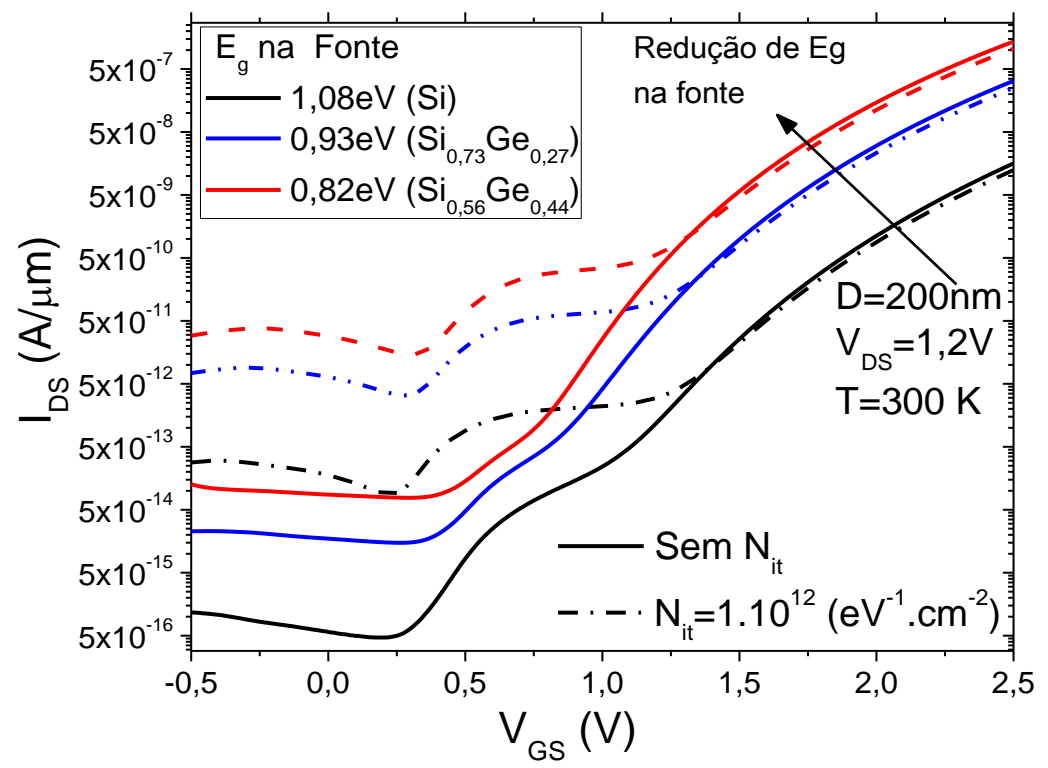

Fonte: Autor

Observa-se que a curva de lds aumenta com a mesma taxa para todos os valores de $\mathrm{V}_{\mathrm{GS}}$ com a diminuição de $\mathrm{E}_{\mathrm{g}}$, assim como observado experimentalmente. Nota-se também que para valores de $\mathrm{V}_{\mathrm{GS}}$ abaixo de 1,2 $\mathrm{V}$, devido a presença de armadilhas de interface ocorre um aumento da corrente de dreno, sendo este aumento devido a influência indireta das armadilhas de interface na geração e recombinação 
$\mathrm{SRH}$ e a influência direta na região entre $0,2 \mathrm{~V}$ e $1,2 \mathrm{~V}$, onde o mecanismo de condução predominante é o TAT. Se o $\mathrm{N}_{\text {it }}$ não for considerado, a componente do TAT é reduzida demonstrando uma melhor inclinação de sublimiar para $\mathrm{V}_{\mathrm{Gs}}$ abaixo de $0.6 \mathrm{~V}$.

A figura 3.26 mostra o comportamento experimental de IDS $\times V_{G S}$ para os dois NW-TFET com fontes de Si e liga $\mathrm{Si}_{0,73} \mathrm{Ge}_{0,27}$, com temperatura variando entre $300 \mathrm{~K}$ e $425 \mathrm{~K}$.

Figura 3.26 - Curvas experimentais de IDS $x V_{G S}$ em função da temperatura para NW-TFET com diferentes materiais de fonte

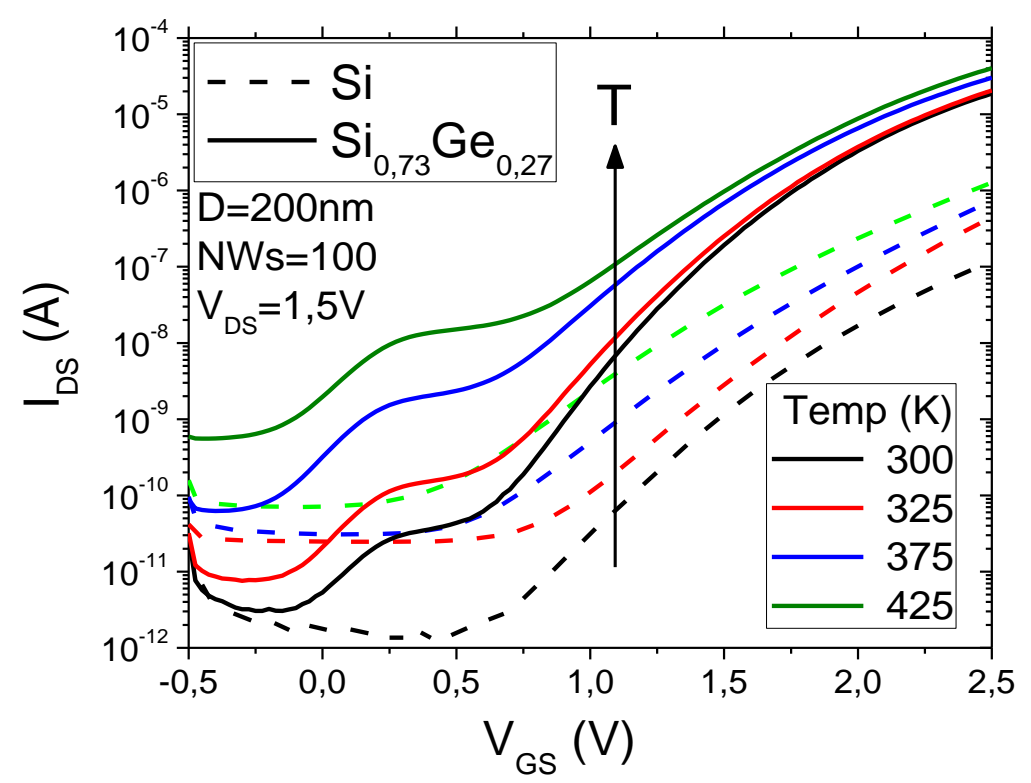

Fonte: Autor

Focando no NW-TFET com fonte de liga SiGe, é possível observar que a elevação da temperatura causa um aumento na corrente de dreno na região de lofF devido à dependência direta da recombinação e geração $\mathrm{SRH}$ com a temperatura, conforme demonstrado na equação 2.9. Observa-se também que a região do platô torna-se mais pronunciada devido à alta influência do $\mathrm{N}_{\text {it, }}$ que enfatiza o tunelamento pelas armadilhas, sendo este aumento ainda mais pronunciado devido à influência da temperatura no TAT, conforme demonstra a equação 2.10. Embora lon apresente também um aumento, este é relativamente menos afetado pela temperatura devido a influência indireta no tunelamento de banda para banda, conforme apresentado na equação 2.11. Dessa forma, o aumento do $\mathrm{N}_{\text {it }}$ provoca maior degradação da taxa de IoN/loff em altas temperaturas. 
Analisando o dispositivo com fonte de silício, é possível observar que as curvas de IDs para diferentes temperaturas mantem-se praticamente paralelas para todos os valores de $V_{G S}$. Conforme apresentado na figura 3.17, isso ocorre devido ao dispositivo não ter atingindo a região onde o tunelamento de banda para banda é o mecanismo de transporte dominante, ou seja, valores de $\mathrm{V}_{\mathrm{Gs}}$ muito acima de $2 \mathrm{~V}$.

A figura 3.27 apresenta a influência do $N_{\text {it }}$ em função da temperatura para dispositivos com fonte de silício. Nesta figura são apresentadas as curvas IDS $\mathrm{x}$ VGS, com $\mathrm{V}_{\mathrm{DS}}=1,2 \mathrm{~V}$, temperatura variando de $300 \mathrm{~K}$ à $475 \mathrm{~K}$ e Nit $=1.10^{12} \mathrm{ev}^{-1} \mathrm{~cm}^{-2}$.

Figura 3.27 - Curvas simuladas de lDS $x V_{G S}$ comparando a influência do $N_{i t}$ na interface fonte/canal em função da temperatura

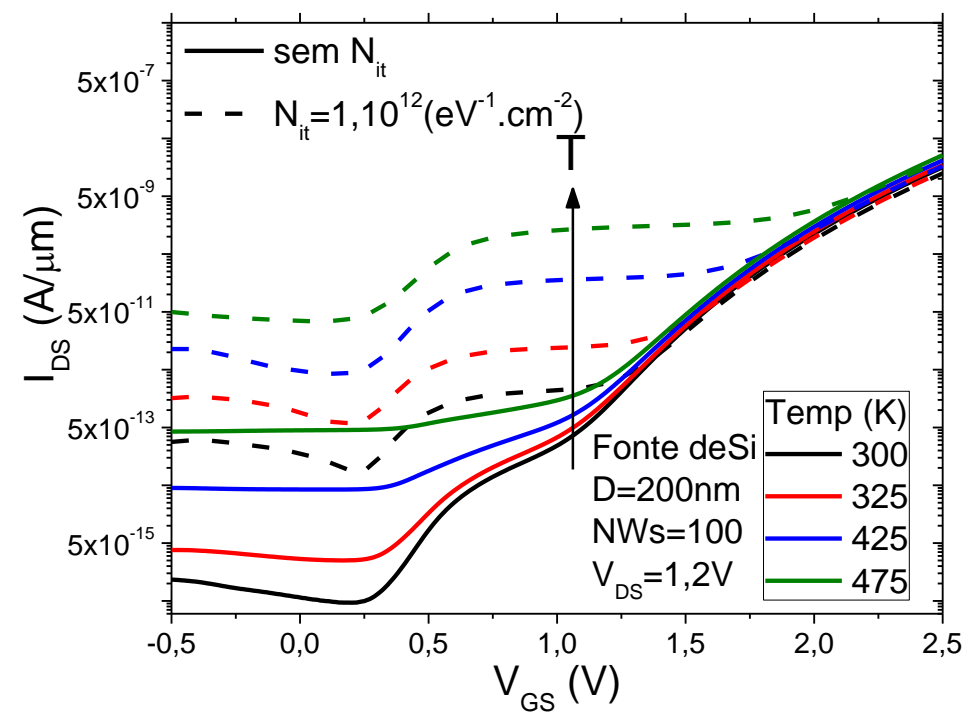

Fonte: Autor

Observava-se que com o aumento da temperatura, a região de loff aumenta e o TAT torna-se predominante, mesmo para altos valores de $V_{G s}$. Quando considerado a densidade das armadilhas, nota-se que com o aumento da temperatura devido ao $\mathrm{N}_{\text {it }}$ e a direta influência da temperatura TAT predomina para quase todos os valores de $V_{\text {Gs. }}$ Para $\mathrm{T}=475 \mathrm{~K}$ o tunelamento de banda para banda, só prevalece sobre 0 TAT para valores acima de $2 \mathrm{~V}$. 


\section{$4 \quad$ NW-TFET COM FONTE DE Ge}

Com o intuito de obter dispositivos com um desempenho ainda melhor do que os com fonte de $\mathrm{Si}_{1-x} \mathrm{Ge}_{\mathrm{x}}$, apresentados no capítulo 3, foram fabricados no Imec, Bélgica transistores NW-TFET com fonte puramente de Ge. Além disso, foram realizadas algumas modificações no processo de fabricação afim de garantir menos defeitos (23).

Ao longo deste capítulo são apresentados alguns detalhes do processo de fabricação dos NW-TFETs com fonte de Ge e os resultados experimentais obtidos, iniciando pelo estudo do comportamento dos NW-TFET com fonte de Ge, a influência da espessura do dielétrico de porta, seguido pelo estudo da influência da temperatura nos parâmetros analógicos e por fim uma análise e modelamento do ruído de baixa frequência para esses transistores.

Todas as análises aqui apresentadas foram realizadas no Imec, Bélgica, durante o estágio de doutorado no exterior com duração de 13 meses.

\subsection{Processo de fabricação}

O processo de fabricação do NW-TFET com fonte de Ge foi realizado no Imec, Bélgica em 2014. Neste novo processo foi empregado o método de substituição, onde uma camada de sacrifício de $\mathrm{Si}_{0,7} \mathrm{Ge}_{0,3}$ policristalino é utilizada na região de fonte, como apresentado na figura 4.1(a) e descrito em (23). Esta alteração no processo garante que o material dielétrico de porta não seja danificado devido a implantação de dopantes, como utilizado no processo anterior, além de garantir um perfil de junção abrupta (52).

As etapas seguintes ocorrem assim como apresentado no capítulo 3.1. Sendo realizada a formação da estrutura de nanofio através do método de litografia por feixe de elétrons (e-beam litography), figura 4.1(b). Em seguida é crescido o óxido inferior, criando um isolamento entre a porta e o substrato, criando o subposicionamento (underlap) da porta na região de dreno, figura 4.1(c). E então é criada a região de porta, com a deposição de uma camada de óxido de háfnio ( $\mathrm{t}_{\mathrm{H}} \mathrm{O}_{2}$ ) com $2 \mathrm{~nm}$ ou $3 \mathrm{~nm}$ sobre uma camada de $1 \mathrm{~nm}$ de óxido de silício, seguida por $5 \mathrm{~nm}$ de nitreto de titânio 
(TiN) como metal de porta e por fim, 30nm de Si amorfo, conforme apresentado na figura figura 4.1 (d). O canal é isolado através de uma camada de óxido, figura 4.1 (e).

Logo após, a camada de sacrifício é totalmente removida com vapor de $\mathrm{HCl}$ e uma camada Ge cristalino (c-Ge) dopado com átomos de Boro e espessura de 0,5 nm a $15 \mathrm{~nm}$ é crescida epitaxialmente. Em seguida a lâmina é transferida à câmara de deposição de vapor químico térmico rápido (Rapid Thermal Chemical Vapor Deposition - RTCVD), onde 100nm de Ge amorfo ( $\alpha-\mathrm{Ge}$ ), também dopado com átomos de Boro, é depositado, conforme apresentado na figura 4.1(f). Para reduzir a resistência de acesso, uma etapa de recozimento térmico é realizada para cristalizar a camada de $\alpha-G e$ na região da fonte.

Para finalizar, o contato de fonte é isolado por espaçadores de nitreto, figura 4.1(g) e as regiões de contato abertas, figura 4.1 (h).

Figura 4.1 - Etapas do processo de fabricação de um vertical NW-TFET com fonte de Ge (23)

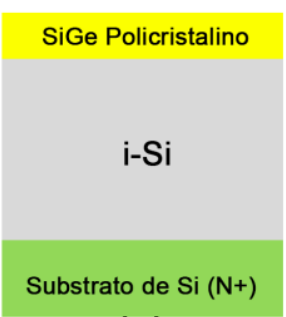

(a)

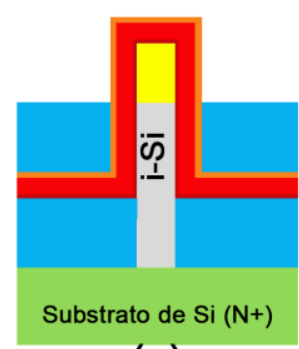

(e)

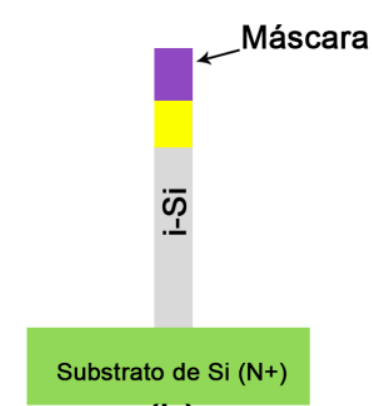

(b)

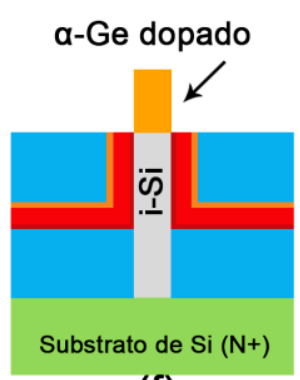

(f)

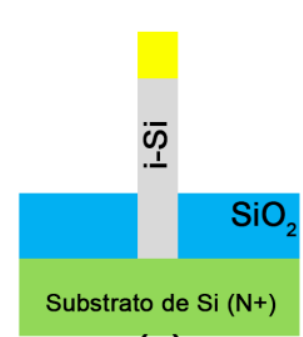

(c)

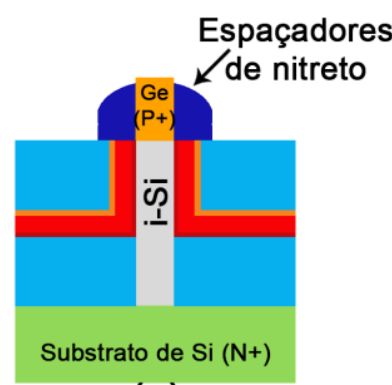

(g)

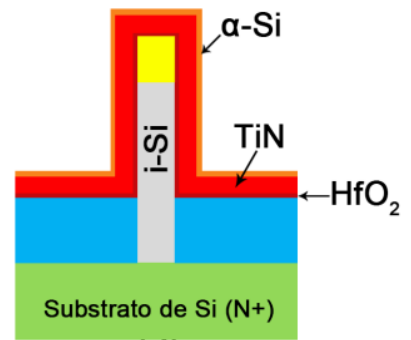

(d)

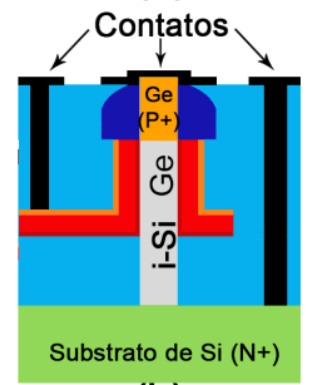

(h)

Fonte: Adaptado de Rooyackers (2013) 


\subsection{Dimensões}

A seção transversal de um NW-TFET está ilustrada na figura 4.2, onde, LcH é o comprimento total de canal, LG o comprimento de porta, LGs a sobreposição (overlap) de porta na região da fonte, LGD a subposição (underlap) de porta na região de dreno e D o diâmetro.

Estes dispositivos apresentam sobreposição de porta na região de fonte, a fim de aumentar a corrente de tunelamento e subposição de porta na região de dreno, para diminuir ou suprimir a corrente ambipolar.

A tabela 4.1 apresenta todas as dimensões de cada uma das lâminas utilizadas nesta sequência de trabalho.

Figura 4.2 - Corte transversal (a) e o Esquemático da seção transversal (b) de um de um NW-TFET
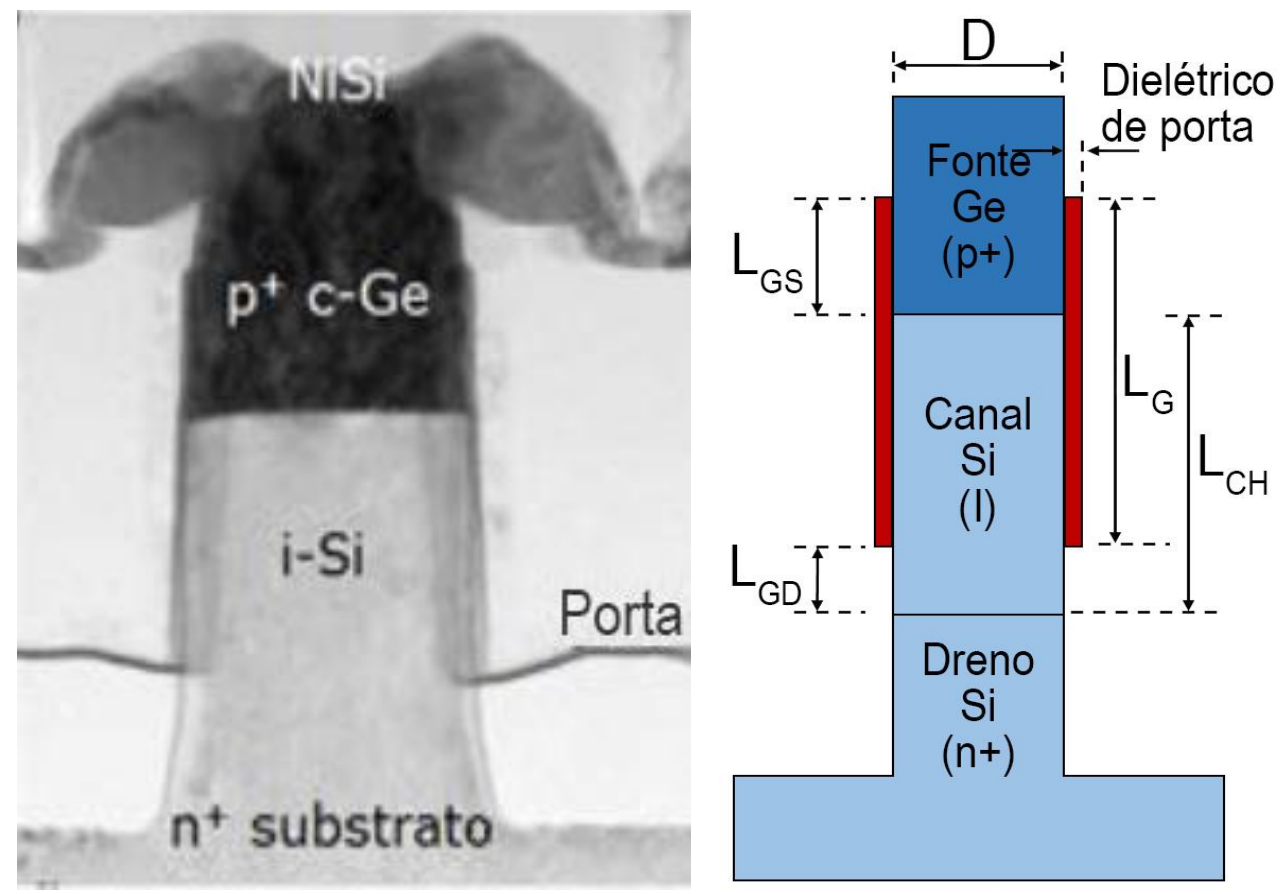

Fonte: Rooyackers (2013) (a) e Autor (b) 
Tabela 4-1 - Dimensões dos dispositivos NW-TFET com fonte de Ge e Si fabricados no Imec, Bélgica e utilizados neste trabalho

\begin{tabular}{|c|c|c|c|c|}
\hline & Lâmina 5 & Lâmina 6 & Lâmina 7 & Lâmina 8 \\
\hline Material da fonte & $\mathrm{Ge}$ & $\mathrm{Ge}$ & $\mathrm{Ge}$ & Si \\
\hline Comprimento de canal ( $\mathrm{LCH})$ & $220 \mathrm{~nm}$ & $220 \mathrm{~nm}$ & $220 \mathrm{~nm}$ & $220 \mathrm{~nm}$ \\
\hline Comprimento de porta $\left(L_{G}\right)$ & $250 \mathrm{~nm}$ & $250 \mathrm{~nm}$ & $250 \mathrm{~nm}$ & $250 \mathrm{~nm}$ \\
\hline Sobreposição da porta/fonte (LGS) & $85 \mathrm{~nm}$ & $85 \mathrm{~nm}$ & $85 \mathrm{~nm}$ & $85 \mathrm{~nm}$ \\
\hline Subposição de porta/dreno ( $\mathrm{L}_{\mathrm{GD}}$ ) & $50 \mathrm{~nm}$ & $50 \mathrm{~nm}$ & $50 \mathrm{~nm}$ & $50 \mathrm{~nm}$ \\
\hline Dopagem de fonte & $1.10^{20} \mathrm{~cm}^{-3}$ & $1.10^{20} \mathrm{~cm}^{-3}$ & $1.10^{20} \mathrm{~cm}^{-3}$ & $1.10^{20} \mathrm{~cm}^{-3}$ \\
\hline Dopagem de canal & $1.10^{16} \mathrm{~cm}^{-3}$ & $1.10^{16} \mathrm{~cm}^{-3}$ & $1.10^{16} \mathrm{~cm}^{-3}$ & $1.10^{16} \mathrm{~cm}^{-3}$ \\
\hline Dopagem de dreno & $2.10^{19} \mathrm{~cm}^{-3}$ & $2.10^{19} \mathrm{~cm}^{-3}$ & $2.10^{19} \mathrm{~cm}^{-3}$ & $2.10^{19} \mathrm{~cm}^{-3}$ \\
\hline Diâmetro (D) & $200 \mathrm{~nm}$ & $200 \mathrm{~nm}$ & $200 \mathrm{~nm}$ & $200 \mathrm{~nm}$ \\
\hline Espessura de Ge cristalino (tc-Ge) & $15 \mathrm{~nm}$ & 0 & $15 \mathrm{~nm}$ & $15 \mathrm{~nm}$ \\
\hline Espessura de $\mathrm{HfO}_{2}\left(\mathrm{tHfO}_{2}\right)$ & $3 \mathrm{~nm}$ & $3 n m$ & $2 \mathrm{~nm}$ & $3 \mathrm{~nm}$ \\
\hline Nanofios em paralelo & 400 ou 5000 & 400 ou 5000 & 400 ou 5000 & 400 ou 5000 \\
\hline
\end{tabular}

Fonte: Autor

\subsection{Parâmetros analógicos do NW-TFET com fonte de Ge e a influência da espessura do óxido de porta}

Como dispositivo de referência foi adotada a lâmina 5, apresentada no capítulo

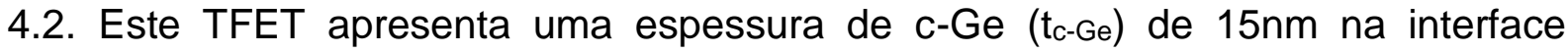
fonte/canal e $3 \mathrm{~nm}$ de $\mathrm{HfO}_{2}$ no material dielétrico de porta. O comportamento analógico deste dispositivo foi comparado com um TFET sem c-Ge (tc-Ge $=0 \mathrm{~nm}$ ), ou seja, apenas a camada de $\alpha-G$ e foi depositada (lâmina 6 ). E outro dispositivo com a mesma espessura de c-Ge, porém com uma camada mais fina de $\mathrm{HfO}_{2}$ (thfor $=2 \mathrm{~nm}$ ) no dielétrico de porta (lâmina 7). A variação em cada um dos dispositivos estudados está sumarizada na tabela 4.2 .

Tabela 4-2 - Diferença entre os dispositivos NW-TFET utilizados neste capítulo

\begin{tabular}{l|c|c|c}
\hline & Lâmina 5 (Ref.) & Lâmina 6 & Lâmina 7 \\
\hline Material da fonte & $\mathrm{Ge}$ & $\mathrm{Ge}$ & $\mathrm{Ge}$ \\
\hline Espessura de Ge cristalino (tc-Ge) & $15 \mathrm{~nm}$ & 0 & $15 \mathrm{~nm}$ \\
Espessura de $\mathrm{HfO}_{2}$ (tHfo2) & $3 \mathrm{~nm}$ & $3 \mathrm{~nm}$ & $2 \mathrm{~nm}$ \\
Nanofios em paralelo (NWs) & 400 & 400 & 400 \\
\hline
\end{tabular}


A figura 4.3 apresenta a curva experimental de IDS $x$ VGS, com VDS variando de $1,1 \mathrm{~V}$ à 1,8 $\mathrm{V}$. Dos resultados obtidos, é possível observar que o dispositivo de referência apresenta maior corrente de funcionamento (ION) quando comparado ao dispositivo sem a camada de c-Ge (lâmina 6). Isso ocorre devido à menor concentração de Boro resultante do processo de fabricação.

O NW-TFET com tHFO2 $_{\text {H }} 2 \mathrm{~nm}$ apresenta menor lDs para menores $\mathrm{V}_{\text {GS }}$ devido ao melhor acoplamento eletrostático entre a porta e o canal, dessa forma, reduzindo o componente de TAT da corrente. Já para valores mais altos de $\mathrm{V}_{\mathrm{GS}}$, a menor espessura de $\mathrm{HfO}_{2}$ resulta em um campo elétrico mais forte, induzindo à melhora do BTBT, o que resulta no chaveamento mais rápido entre os estados ligado/desligado (on/off), explicando a menor inclinação de sublimiar (SS) observada na figura 4.3.

Ao comparar o dispositivo com menor espessura de $\mathrm{HfO}_{2}$ (lâmina 7) com o de referência, nota-se que este apresenta um pequeno aumento de lon e inclinação de sublimiar mais íngreme. Esta melhora pode ser atribuída ao melhor acoplamento eletrostático entre a fonte e o canal, devido ao aumento da corrente de tunelamento nesta região.

Além disso, é possível observar que independente da tensão aplicada ao dreno, não houve variação no nível de lDs para tensões de porta (VGS) mais altas devido a saturação de tunelamento.

As curvas de corrente de dreno (IDS) em função da tensão aplicada ao dreno (VDS) para os três dispositivos estão plotadas na figura 4.4. Verifica-se que todos os dispositivos apresentam uma região de platô, que se assemelha à região de saturação do MOSFET convencional. 
Figura 4.3 - Curvas experimentais de IDS $\times V_{\text {GS }}$ para NW-TFETs com diferentes composições de fonte e espessura de $\mathrm{HfO}_{2}$ no dielétrico de porta

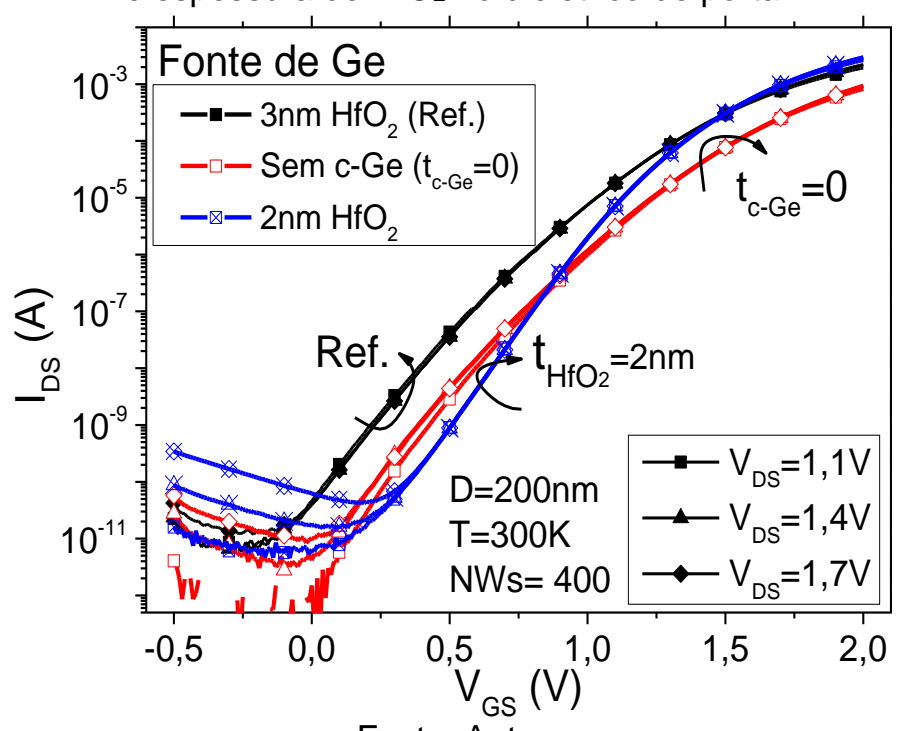

Fonte: Autor

Figura 4.4 - Curva de lDS $x$ VDS para NW-TFETs com diferentes composições de fonte e espessura de $\mathrm{HfO}_{2}$ no dielétrico de porta
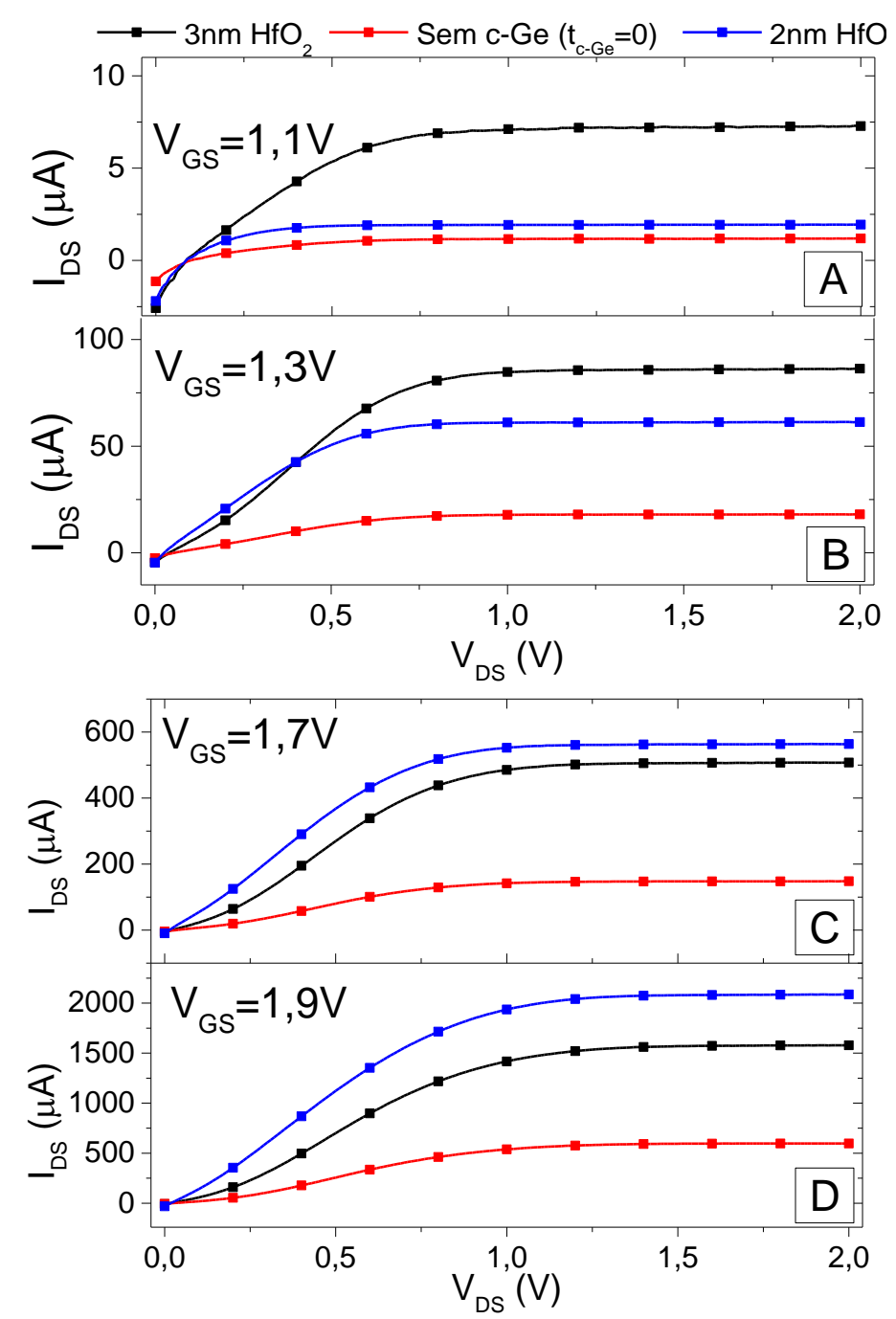

Fonte: Autor 
O dispositivo sem a camada C-Ge apresenta o menor valor de IDs para todos os valores de tensão porta aplicados. Entretanto, o dispositivo de referência, quando comparado ao com menor espessura de $\mathrm{HfO}_{2}$, apresenta maior nível IDS para $V_{G S}<1,5 \mathrm{~V}$, figura 3 (a) e (b), e menor lDs para $V_{G S}>1,5 \mathrm{~V}$, figura 3 (c) e (d).

Os valores de $g_{m}$ obtidos experimentalmente estão plotados em função de $V_{G s}$ na figura 4.5. Observa-se que o dispositivo sem a camada de c-Ge apresenta o mesmo comportamento que o dispositivo de referência, porém com um nível de gm reduzido em uma ordem de grandeza.

Os dispositivos de referência e o com espessura mais fina de $\mathrm{HfO}_{2}$ apresentam valores similares de $\mathrm{gm}_{\mathrm{m}}$ em $\mathrm{V}_{\mathrm{GS}}=1,3 \mathrm{~V}$. Entretanto, para $\mathrm{V}_{\mathrm{GS}}<1,3 \mathrm{~V}$, os dispositivos com espessura mais fina de $\mathrm{HfO}_{2}$, devido à redução da influência de TAT, apresentam menores valores de corrente e como consequência, menores valores de $g_{m}$, quando comparado ao dispositivo de referência nesta condição de polarização. Porém, para VGS $>1,3 \mathrm{~V}$ apresenta maior nível de $\mathrm{gm}_{\mathrm{m}}$ devido à melhora do BTBT com o aumento do campo elétrico.

Figura 4.5 - Curva experimental $g_{m} \times V_{G S}$ para NW-TFETs com diferentes composições de fonte e espessura de $\mathrm{HfO}_{2}$ no dielétrico de porta

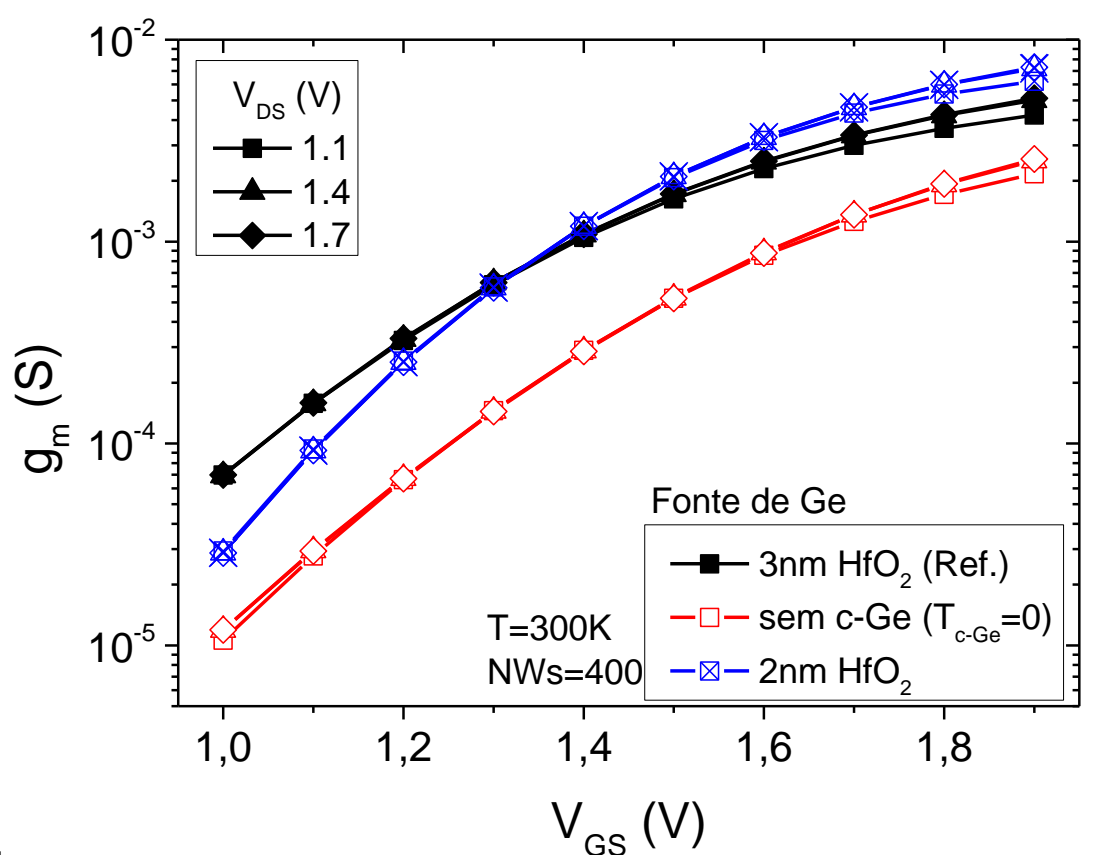

Fonte: Autor 
As curvas de condutância de saída ( $\left.g_{d}\right)$ em função da tensão aplicada a porta (VGS) estão apresentados na figura 4.6. Nesta figura, nota-se que devido ao menor valor de IDS, o dispositivo sem a camada de c-Ge apresenta melhor gd para todas as condições de polarização quando comparado ao dispositivo de referência. Entretanto, o dispositivo com espessura fina de $\mathrm{HfO}_{2}$ apresenta melhor $g_{d}$ para $\mathrm{V}_{\mathrm{GS}}=1,1 \mathrm{~V}$ devido à redução da influência de TAT e tendendo ao mesmo valor de gd para $V_{G S}>1,8 \mathrm{~V}$, onde o BTBT atinge a taxa máxima de tunelamento. Além disso, é possível observar que em todos os casos, gd degrada com o aumento de VGs.

Figura 4.6 - Curvas experimentais de $g_{d} \times V_{G S}$ para NW-TFETs com diferentes composições de fonte e espessura de $\mathrm{HfO} 2$ no dielétrico de porta

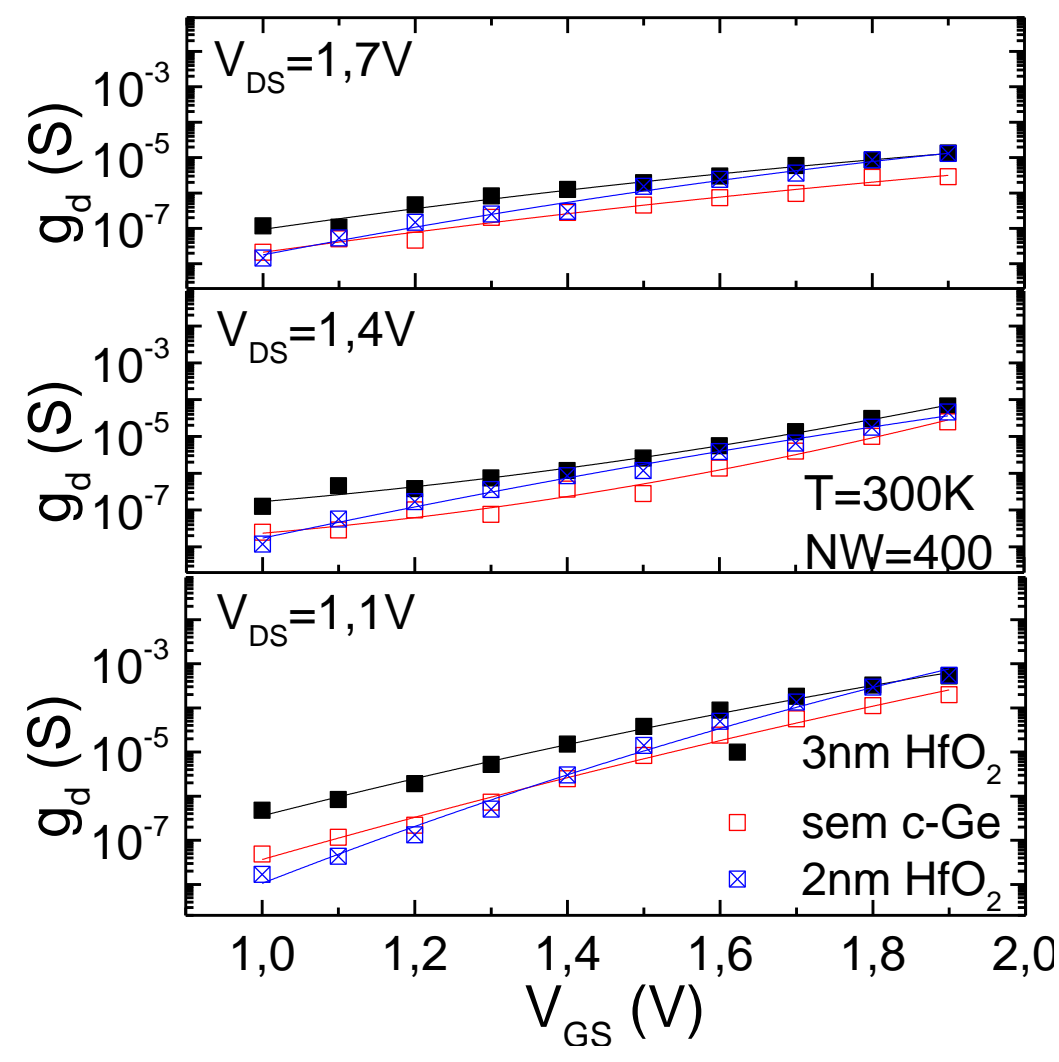

Fonte: Autor

A figura 4.7 apresenta as curvas de $A v$ em função de $V_{G S}$ para todos os três dispositivos. Desta figura, é possível observar que o TFETs sem a camada de c-Ge e o com menor espessura de $\mathrm{HfO}_{2}$ apresentaram maior $\mathrm{Av}$ que o dispositivo de referência, indicando que $g_{d}$ é um fator dominante para todos os transistores analisados. Ao comparar a variação da tensão aplicada à porta, nota-se a degradação de $A v$ com o aumento de $V_{G s}$, também induzido pela degradação de gd com o aumento de VGS. 
Considerando $\mathrm{V}_{\mathrm{GS}}=1,1$ e $\mathrm{V}_{\mathrm{DS}}=1,0 \mathrm{~V}$, o dispositivo sem a camada de $\mathrm{c}-\mathrm{Ge}$ apesenta $A v$ em torno de 4,3 dB maior que o dispositivo de referência, enquanto 0 com menor espessura de $\mathrm{HfO}_{2}$ apresenta $21,4 \mathrm{~dB}$ a mais. Para $\mathrm{V}_{\mathrm{GS}}=1,8 \mathrm{~V}$ e $\mathrm{V}_{\mathrm{DS}}=$ $1,0 \mathrm{~V}$, quando comparado ao dispositivo de referência, esta diferença diminui para 2,7 dB para o TFET sem a camada de c-Ge e 2,9 dB para o com menor thfo2. Isto ocorre devido a variação de $g_{d}$ com $V_{G s}$ como demonstrado anteriormente. Esta variação de $g_{d}$ é resultante do deslocamento da região de saturação com $V_{G S}$, como observado na figura $4.4(\mathrm{a})$, considerando $\mathrm{V}_{\mathrm{GS}}=1,0 \mathrm{~V}$, observa-se que $\mathrm{V}_{D S}>1,0 \mathrm{~V}$ encontra-se na região de saturação, enquanto que nas figuras 4.4 (c) e (d), onde $V_{G S}=1.7 \mathrm{~V}$ e 1.9V, respectivamente, o platô de saturação desloca-se para valores mais altos de $\mathrm{V}_{\mathrm{DS}}$, resultando no aumento de $g_{d}$ e como consequência, diminuição de Av.

Figura 4.7 - Curvas experimentais de $A v \times V_{G s}$ para NW-TFETs com diferentes composições de fonte e espessura de $\mathrm{HfO}_{2}$ no dielétrico de porta

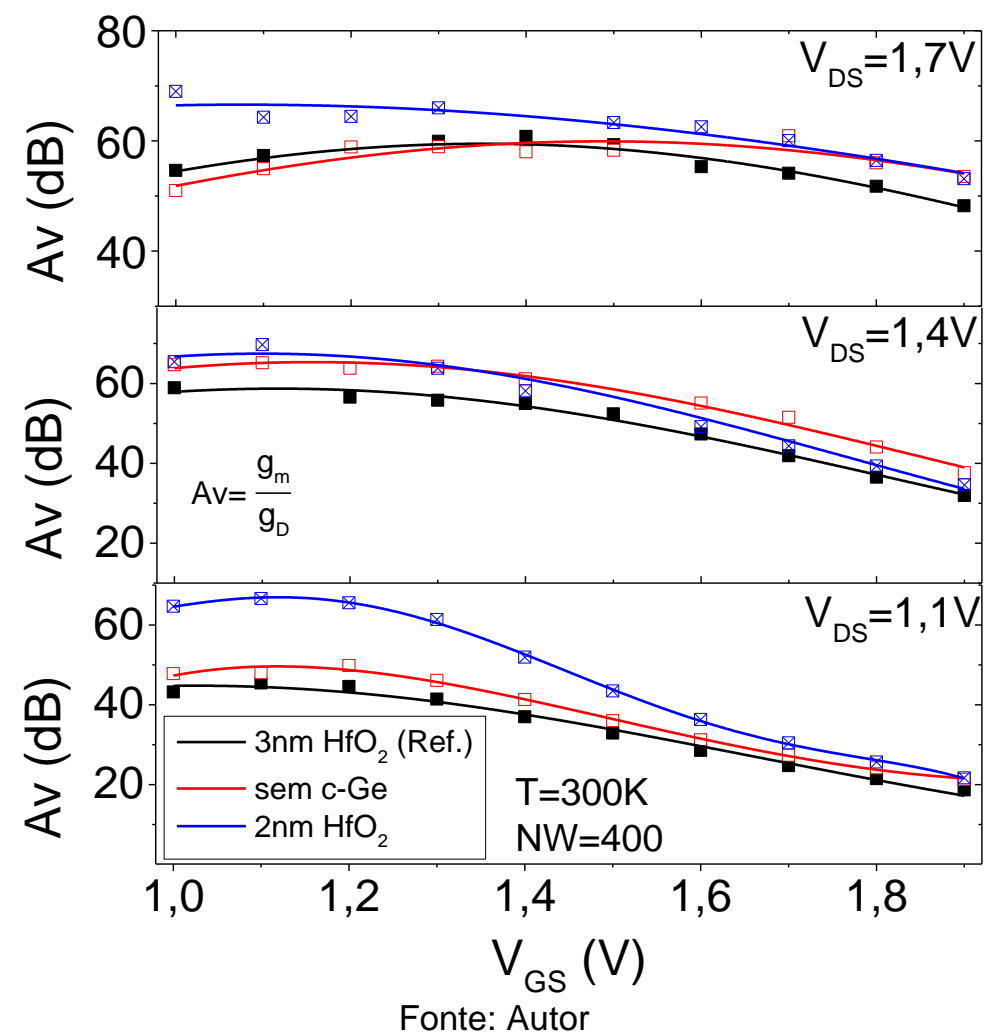

Um ponto importante a ser destacado são os altos valores de Av atingidos, sendo observado valores em torno de 60 à $65 \mathrm{~dB}$ para os três TFETs com VDS 1,4 V e $V_{G S}=1,1 \mathrm{~V}$, reforçando que apesar destes transistores terem sido desenvolvidos com o intuito de aplicações digitais, estes estão apresentando bons resultados analógicos. 


\subsection{Estudo de ruído de baixa frequência em transistores NW-TFET}

Nesta parte do trabalho foi investigado o impacto da heterojunção e da espessura de $\mathrm{HfO}_{2}$ no dielétrico de porta na densidade espectral de ruído nos transistores NW-TFET.

Até o presente momento não existem modelos propostos para o estudo de ruído em TFETs, por isso serão usados os modelos propostos para transistores MOSFETs e comparados os resultados. Para que seja compatível com os estudos de MOSFETs existentes, todos os resultados aqui apresentados serão normalizados pela circunferência e pelo número de nanofios em paralelo ( $\left.\pi^{\star} D^{\star} N W s\right)$.

Para este estudo foram utilizadas as lâminas 5, 7 e 8 apresentado no capítulo 4.2 e suas dimensões na tabela 4.1. Sendo estes, dois NW-TFETs com fonte de Ge e espessura de $\mathrm{HfO}_{2}$ de $3 \mathrm{~nm}$ (referência) e $2 \mathrm{~nm}$. E um com fonte de Si e thfO2 $=3 \mathrm{~nm}$. Em todas as medidas foram utilizadas cascatas com 5000 nanofios em paralelo.

Com o intuito de comparar a influência do material de fonte no desempenho do dispositivo, a figura 4.8 apresenta a curva experimental de IDs em função de VGS, com $\mathrm{V}_{\mathrm{DS}}$ variando de $0,9 \mathrm{~V}$ à $1,5 \mathrm{~V}$ e em temperatura ambiente para os três dispositivos descritos na tabela 4.1.

Conforme já observado, devido ao menor $E_{g}$, e consequentemente maior tunelamento de banda para banda, os dispositivos com fonte de Ge apresentam loN com cerca de duas décadas acima, quando comparado ao NW-TFET com fonte de Si. E conforme também já apresentado no capítulo 4.3, o dispositivo com thfO2 $=2 \mathrm{~nm}$ possui melhor acoplamento eletrostático, resultando em melhor controle da porta sobre o canal, o que induz à melhor inclinação de sublimiar.

A fim de melhor visualizar quais os mecanismos de condução ativos em cada uma das polarizações deste transistor, o gráfico de ativação de energia $\left(E_{A}\right)$, extraído pelo método de Arrhenius (39-40), está plotado na figura 4.9. Observa-se que nos dois dispositivos com fonte de Ge o BTBT passa a ser o mecanismo de condução predominante $\left(E_{A}<0,1 \mathrm{eV}\right)$ para $V_{G S}>1,3 \mathrm{~V}$, enquanto isso ocorre apenas em $V_{G S}$ acima de1,9 V no dispositivo o com fonte de Si. 
Figura 4.8 - Curvas experimentais de lDS $x V_{G S}$ para os diferentes NW-TFETs estudados, sendo dois com fonte de $\mathrm{Ge}$ e diferentes espessuras de $\mathrm{HfO}_{2}$ e um com fonte de $\mathrm{Si}$

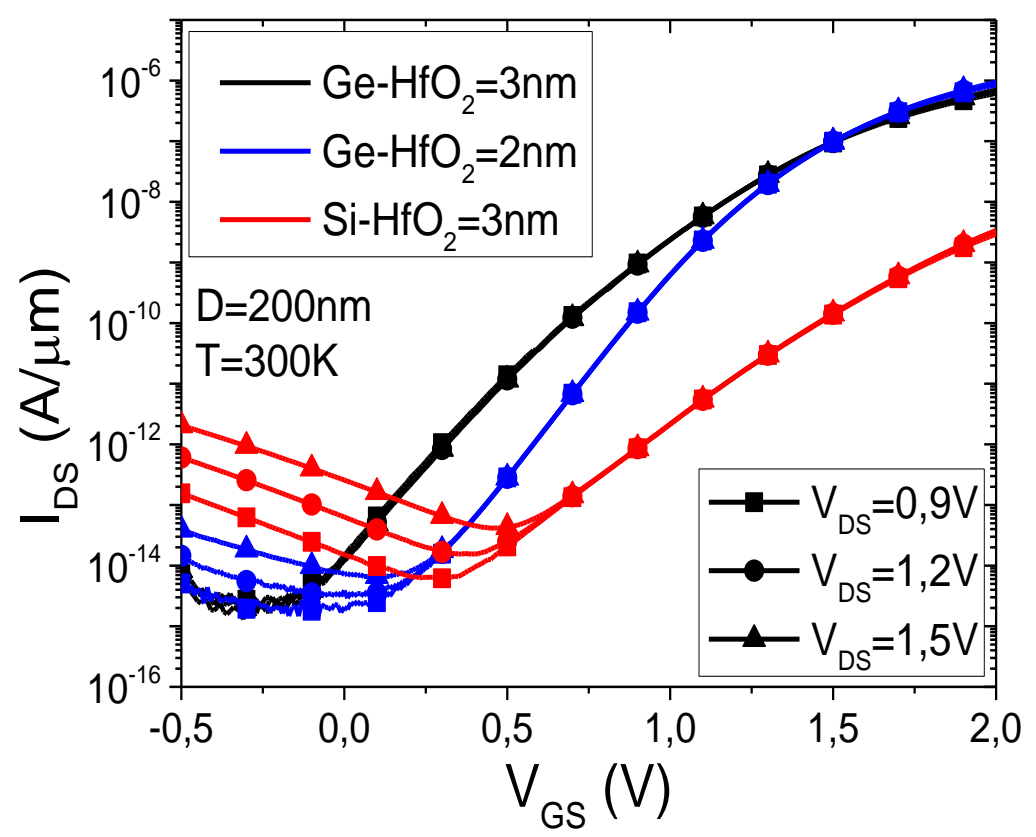

Fonte: Autor

Figura 4.9 - Curvas de $E_{A} \times V_{G S}$ para os diferentes NW-TFETs estudados

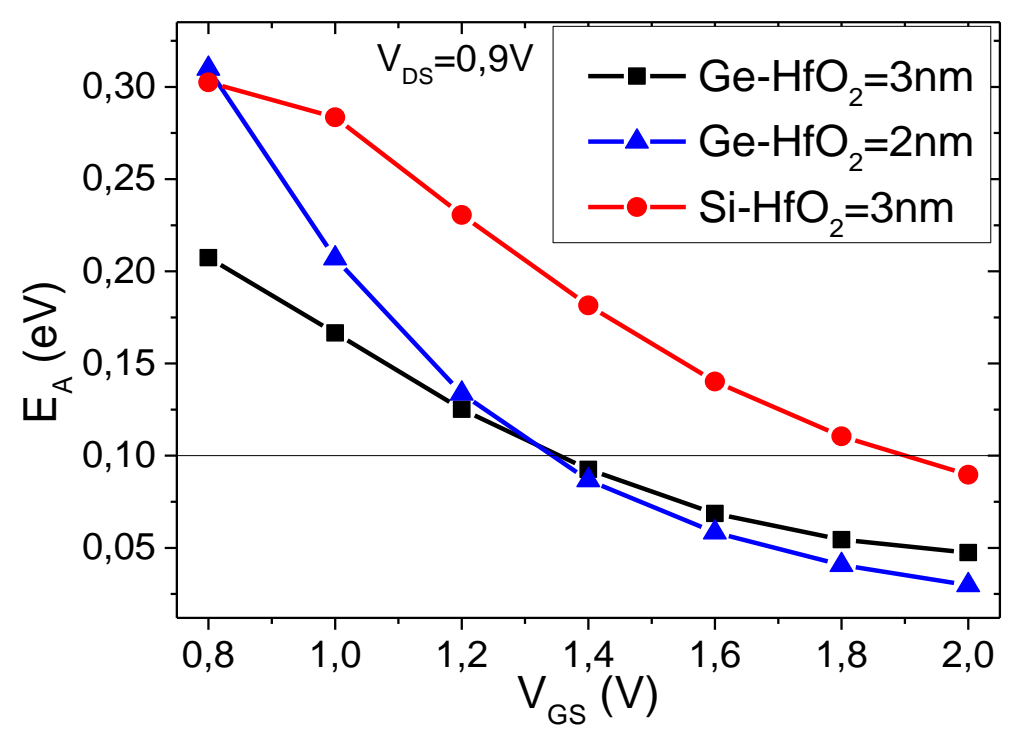

Fonte: Autor 
O mecanismo de transporte de portadores no canal de um transistor é um processo estocástico, ou seja, pode ser perturbado por flutuações randômicas com o tempo. O sinal desejado torna-se difícil de ser distinguido quando o valor do ruído é significante em relação ao sinal, por isso o ruído é indesejado em sistemas eletrônicos (52).

O estudo de ruído é baseado em métodos matemáticos da teoria da probabilidade, que permite definir médias apropriadas das variáveis randômicas que estão sendo tratadas. O método utilizado neste trabalho foi o de descrever o ruído através da conversão do mesmo do domínio do tempo para o domínio da frequência por transformada de Fourier (42). A densidade espectral de potência (Power Spectral Density - PSD) dá informação sobre como a potência do ruído é distribuída na frequência.

A densidade espectral de ruído da corrente de dreno (current noise power spectral density - SID) em função da frequência para o transistor de referência está apresentada na figura 4.10.

Observa-se que para valores de tensões de porta mais baixas ( $\mathrm{V}_{\mathrm{GS}}=0,3 \mathrm{~V}$ ), a curva de SID apresenta uma redução com o aumento de f em uma proporção de 1/f² . Este comportamento, é característico para ruído de geração e recombinação ( $g-r)$ em semicondutores, também conhecido por Lorentzian. Origina-se do armadilhamento randômico de cargas, causando flutuações no número de portadores disponíveis para o transporte de corrente.

Com o aumento da tensão para valores de $\mathrm{V}_{\mathrm{Gs}}$ maiores que $0,7 \mathrm{~V}$, essa diminuição de $S_{I D}$ ocorre na proporção de $1 / f^{\vee}$, sendo $Y$ próximo a 1 . Este comportamento é conhecido como ruído Flicker, com $\mathrm{Y}$ usualmente no intervalo de 0,7 a 1,3. Este ruído é modelado por dois diferentes mecanismos em um transistor MOSFET: flutuações no número de portadores e flutuações na mobilidade (42) 
Figura 4.10 - Curvas experimentais de $S_{I D}$ x $f$ e diferentes polarizações de $V_{D S}$ para um NW-TFET com fonte de $\mathrm{Ge}$

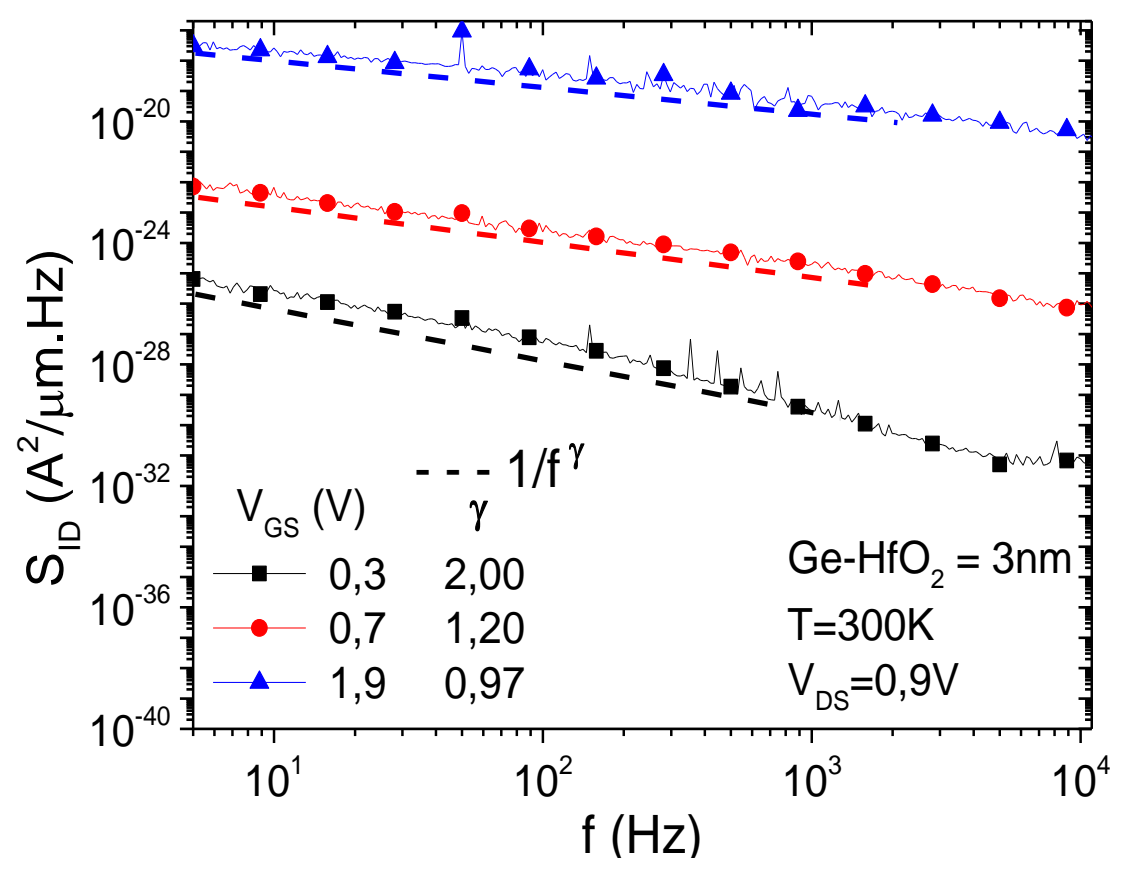

Fonte: Autor

Neste primeiro modelo, proposto por McWhorter (43), as flutuações na corrente acontecem devido ao armadilhamento e desarmadilhamento das cargas no óxido de porta. Enquanto o segundo, proposto por Hooge (44), considera que o ruído 1/f é devido a flutuações na mobilidade.

Para os transistores TFET o ruído Flicker é considerado predominantemente devido ao armadilhamento e desarmadilhamento de cargas na região do dielétrico de porta, próximo à interface na região da fonte.

A densidade espectral de corrente para o MOSFET é descrita pela equação 4.1 (42). 


$$
\begin{gathered}
S_{I D}=I_{D S}^{2} \frac{\beta^{2}}{S S^{2}} \cdot S_{V G}=g_{m}^{2} \cdot S_{V G} \quad \operatorname{com} \beta=k \cdot T \\
S_{V G}=\frac{q^{2} k T N_{o t}}{C_{o x}^{2} W L_{e f f} \alpha f^{\gamma}} \\
I_{D S}=I_{\text {tunneling }}=A_{B T B T} \cdot F^{2} \exp \left(\frac{-B_{B T B T}}{F}\right)
\end{gathered}
$$

onde, $\mathrm{C}_{\mathrm{ox}}$ é a capacitância do óxido, $\alpha$ o parâmetro de tunelamento $\left(\approx 10^{-8} \mathrm{~cm}\right), \mathrm{W}$ a largura do transistor, Leff o comprimento efetivo de canal, $f$ a frequência, $F$ o campo elétrico, $A_{B T B T}$ e $B_{B T B T}$ os parâmetros de ajuste da taxa de geração de tunelamento.

A figura 4.11 apresenta as curvas de SID para baixas frequências $(f<10 \mathrm{~K} \mathrm{~Hz}$ ) para dois TFETs com fonte de Ge e diferentes espessuras de $\mathrm{HfO}_{2}(2 \mathrm{~nm}$ e $3 \mathrm{~nm})$ e um com fonte de Si. Para que a comparação seja válida, a densidade espectral de corrente (SID) para cada um foi extraída considerando um valor fixo de corrente ( IDS $\left.=10^{-11} \mathrm{~A} / \mu \mathrm{m}\right)$, garantindo assim, que todos estejam em uma região dominada pelo TAT.

Figura 4.11 - Curvas de SID x f para os três diferentes NW-TFETs estudados

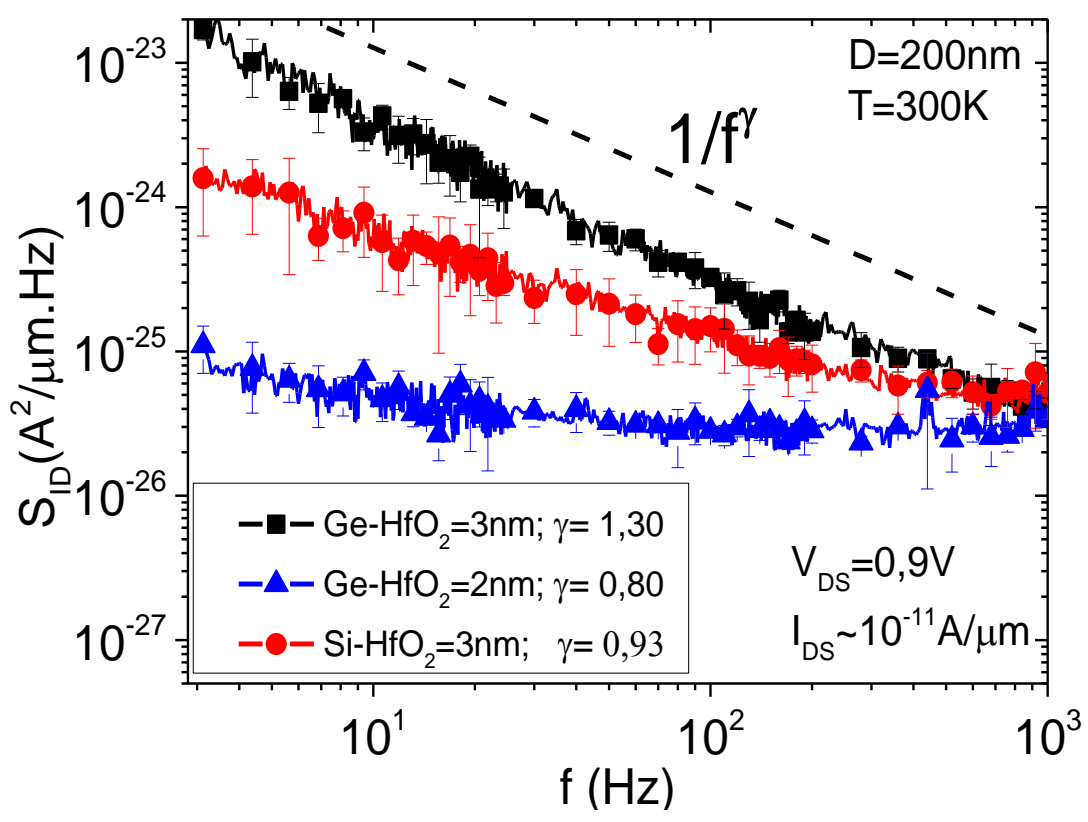

Fonte: Autor 
Conforme já observado para o dispositivo de referência nesta condição de polarização, os outros dois transistores também apresentam SID com um comportamento de 1/f^ , com $\mathrm{y}$ variando de 0,7 a 1,3.

Quando comparado os transistores com fonte $\mathrm{Ge}$ e Si, os dois com thfO2 $=3 \mathrm{~nm}$, observa-se que a presença de Ge na fonte elevou o nível de $S_{I D}$, indicando um aumento na densidade de armadilhas no óxido $\left(\mathrm{N}_{\mathrm{ot}}\right)$, ocorrendo devido a segregação de germânio na camada de Si do óxido de porta durante o processo de fabricação. Entretanto, nota-se que devido a diminuição da espessura de $\mathrm{HfO}_{2}$, ocorre a diminuição de $\mathrm{N}_{\text {ot, }}$ resultando em um menor nível de SID. $_{\text {ID }}$

Com o intuito de verificar se os resultados experimentais seguem o modelo de ruído, foi obtido o SID para frequência de $25 \mathrm{~Hz}$ em função da corrente de dreno e plotado na figura 4.12. Nota-se que o $S_{I D}$ de todos os dispositivos segue a tendência de IDS $^{2}$, estando em acordo com a equação 4.1 .

Figura 4.12 - Curvas de $S_{I D} \times$ IDS extraídas em $f=25 \mathrm{~Hz}$ e $V_{D S}=0,9 V$ para os diferentes NW-TFETS estudados

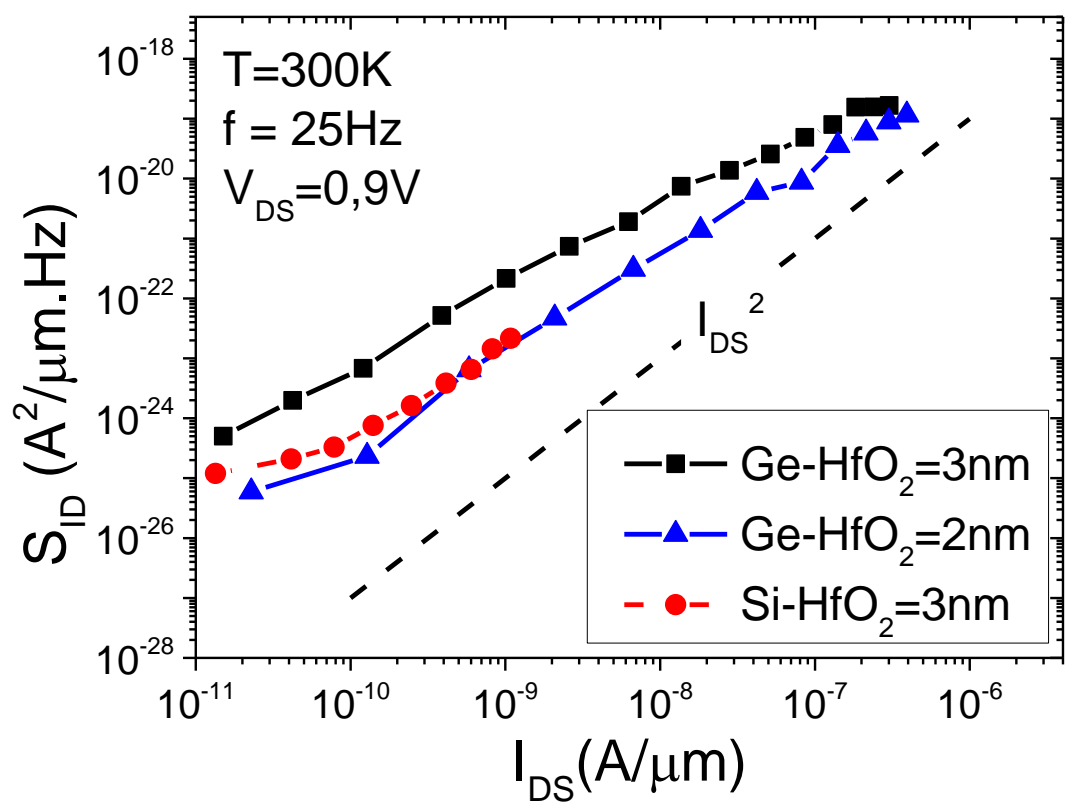

Fonte: Autor 
A figura 4.13 apresenta a curva do SID normalizado por $\operatorname{lDS}^{2}$ (SID/ IDS ${ }^{2}$ ), em função de SS, de acordo com a equação 4.1 o $\left(\mathrm{S}_{\mathrm{ID}} / \mathrm{IDS}^{2}\right)$ deve seguir a tendência de 1/SS ${ }^{2}$. É possível observar que para o TFET com fonte de Ge e tHfO2 $=3 \mathrm{~nm}$, o $\mathrm{SID}_{\mathrm{D}} / \mathrm{IDS}^{2}$ apresenta uma variação de $1 / \mathrm{SS}^{2}$ para valores mais altos de SS. Entretanto o TFET com fonte de $\mathrm{Ge}$ e thfO2 $=2 \mathrm{~nm}$, devido ao melhor acoplamento eletrostático, e como consequência, melhora da corrente de tunelamento (BTBT), apresentou uma variação seguindo a tendência de $1 / S^{2}$ para quase toda faixa de SS analisada.

Figura 4.13 - Curvas de SID normalizados por $\operatorname{lDS}^{2} \mathrm{x}$ inclinação de sublimiar (SS) para NW-TFETs com fonte de $\mathrm{Ge}$

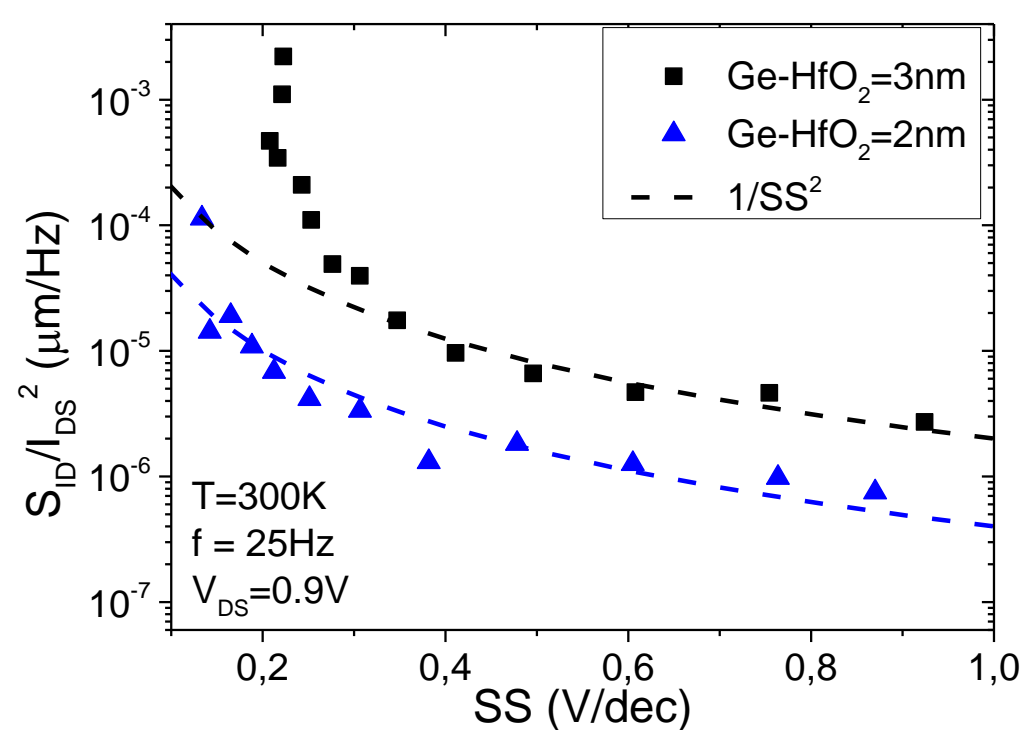

Fonte: Autor

A figura 4.14 apresenta o SID normalizado por $\operatorname{IDS}^{2}\left(S_{I D} / \operatorname{IDS}^{2}\right)$ e a curva de $\left(g_{m} / \mathrm{lDS}\right)^{2}$, ambas com $f=25 \mathrm{~Hz}$ e em função de lDs. Nos estudos de ruído em MOSFETS, o SID normalizado por $\mathrm{IDS}^{2}$ é um parâmetro importante, sendo utilizado para identificar a origem do comportamento do ruído. Observa-se nesta figura que $\mathrm{SID}_{\mathrm{D}} / \mathrm{IDS}^{2}$ diminui continuamente com o aumento de IDS, indicando que a origem deste ruído pode ser atribuída a flutuações no número de portadores.

Comparando com o comportamento conhecido dos MOSFETS, a curva de $\mathrm{SID} / \mathrm{IDS}^{2}$ deveria seguir a mesma tendência que a curva de $\left(\mathrm{g} \mathrm{m} / \mathrm{lDS}_{\mathrm{DS}}{ }^{2}\right.$, porém nota-se que, $\left(g_{m} / l_{D S}\right)^{2}$ não apresenta a mesma tendência de diminuição de forma linear, reduzindo proporcionalmente mais para maiores valores de IDS. 
Figura 4.14 - Curvas experimentais de $S_{I D}$ normalizada por $\operatorname{lDS}^{2}$ e de $\left(g_{m} / I_{D S}\right)^{2} x I_{D S}$ para os diferentes NW-TFETs estudados

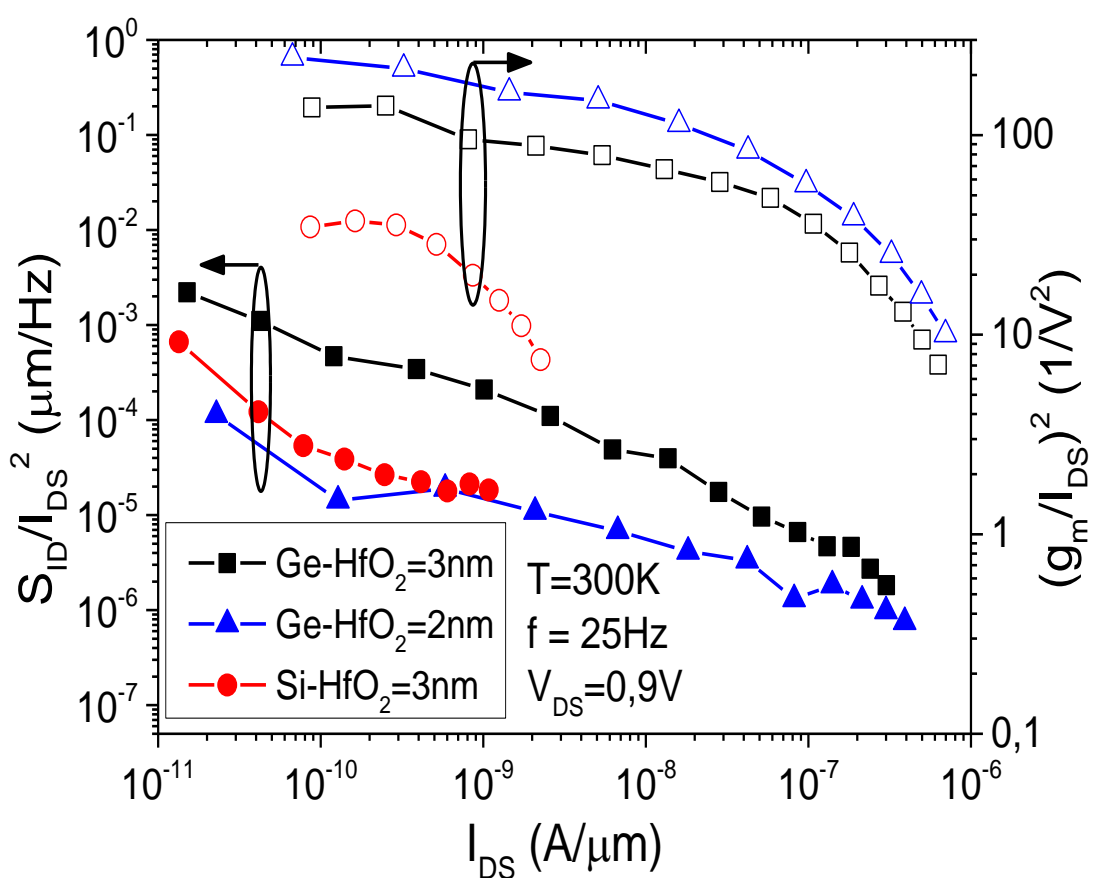

Fonte: Autor

Diferentemente dos MOSFETS, os transistores TFET não apresentam degradação da transcondutância, mesmo para altos valores de $V_{G S}$, devido ao mecanismo de condução predominante ser o BTBT, como pode ser observado na figura 4.15, explicando a diferença no comportamento $\left(\mathrm{gm}_{\mathrm{m}} / \mathrm{lDs}\right)^{2}$ para os TFETs.

Figura 4.15 - Curvas de $g_{m} \times$ VGS para os diferentes NW-TFETs

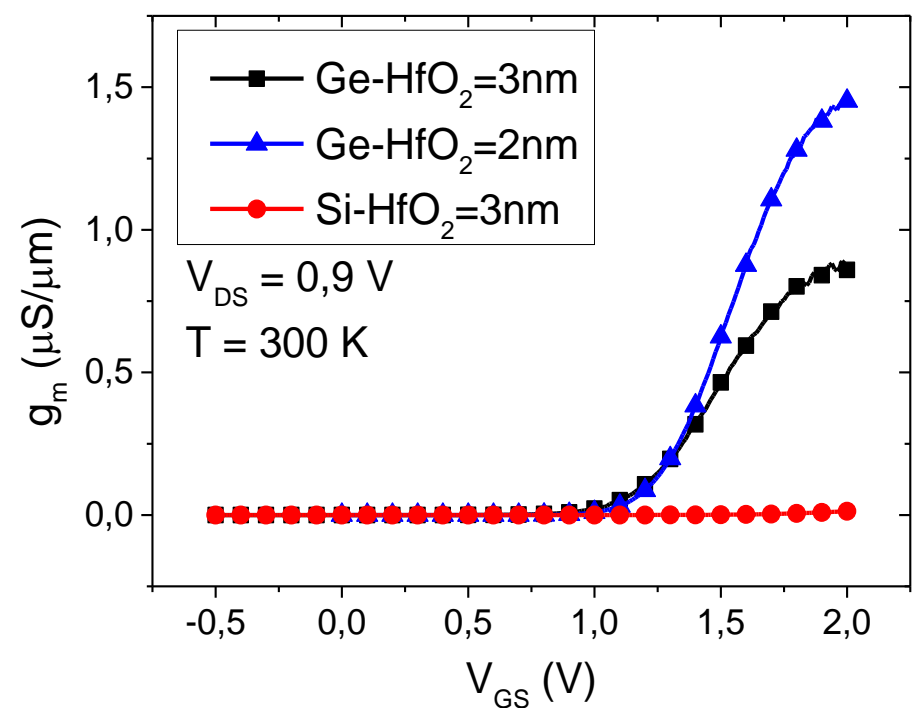

Fonte: Autor 
Outro parâmetro importante para o estudo de ruído nos MOSFETs é a densidade espectral de ruído referenciado a entrada (input referred noise power spectral density - SvG). Este parâmetro é utilizado para extrair a densidade efetiva de armadilhas no oxido de porta $\left(\mathrm{N}_{\mathrm{ot}}\right)$, sendo dado pela equação 4.2 .

A ausência da degradação de $g_{m}$ afeta diretamente o SvG, determinado pela relação $S_{I D} / g_{m}{ }^{2}$. Para um MOSFET a curva $S_{V G} \times V_{G S}$, apresenta tipicamente um valor constante para baixos valores de $\mathrm{V}_{\mathrm{GS}}$, seguido por um aumento para $\mathrm{V}_{\mathrm{Gs}}$ mais altos. Para os TFETs um comportamento totalmente oposto foi observado, como pode ser visto na figura 4.16. Este comportamento inverso pode ser explicado pelos diferentes mecanismos de condução do TFET.

Figura 4.16 - Curvas de SvG $\times$ VGs para $f=25 \mathrm{~Hz}$ e VDS = 0,9V para três diferentes dispositivos estudados

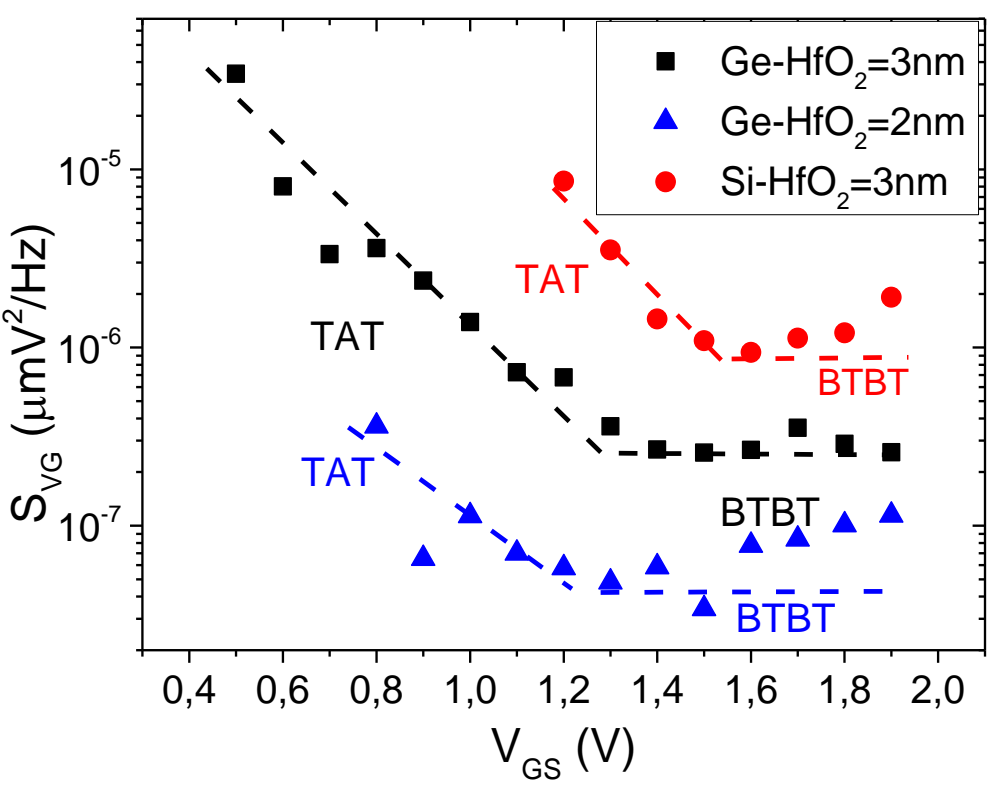

Fonte: Autor

Todos os três dispositivos apresentam uma curva de Svg composta por uma região inclinada, em uma região onde o mecanismo de condução predominante é o TAT, seguido por um platô onde o mecanismo predominante é o BTBT. Considerando os regimes definidos através das curvas de energia de ativação $\left(E_{A}\right)$, apresentada na figura 4.9, observa-se que este comportamento concorda com o observado na curva de SvG, onde a região de platô para os dispositivos com fonte de Ge inicia-se para $V_{G S}>1,3$ e no dispositivo com fonte de Si para $V_{G S}>1,5 \mathrm{~V}$ 
Além disso, nota-se na equação 4.2 que Svg apresenta uma dependência direta com $g_{\mathrm{m}}$, levando a uma inversão nos níveis de ruído quando analisado, onde o TFET com fonte de Si apresenta valores mais altos quando comparado com o de fonte de Ge, enquanto o dispositivo com fonte de Ge e menor thfO2 mantém-se com menor nível de ruído.

\subsubsection{Modelamento do ruído}

Em uma primeira aproximação o modelo de SID para MOSFET será usado para TFET. A fim de torna-lo compatível, o comprimento efetivo do canal (Leff) da equação 4.1 será substituído pelo comprimento efetivo de tunelamento $\left(L_{T}\right)$. $O L_{T}$ foi obtido através de simulações numéricas realizadas no TCAD Silvaco, seguindo o modelo proposto em (53)

A figura 4.17 apresenta a curva de IDS em função de $V_{G S}$ para o TFET referência. Os resultados simulados foram obtidos através do ajuste empírico dos parâmetros $A_{\text {BTBT }}$ e $B_{B T B T}$ da equação 4.3 , sendo eles, $1,22.10^{20} \mathrm{~cm}^{-1} \mathrm{~V}^{-2} \mathrm{~s}^{-1}$ e $1,64.10^{7}$ $\mathrm{V} / \mathrm{cm}$, respectivamente (53).

Figura 4.17 -Curvas de lDS $\times V_{\text {GS }}$ obtidas de forma experimental e simuladas para um NW-TFET com fonte de $\mathrm{Ge}$

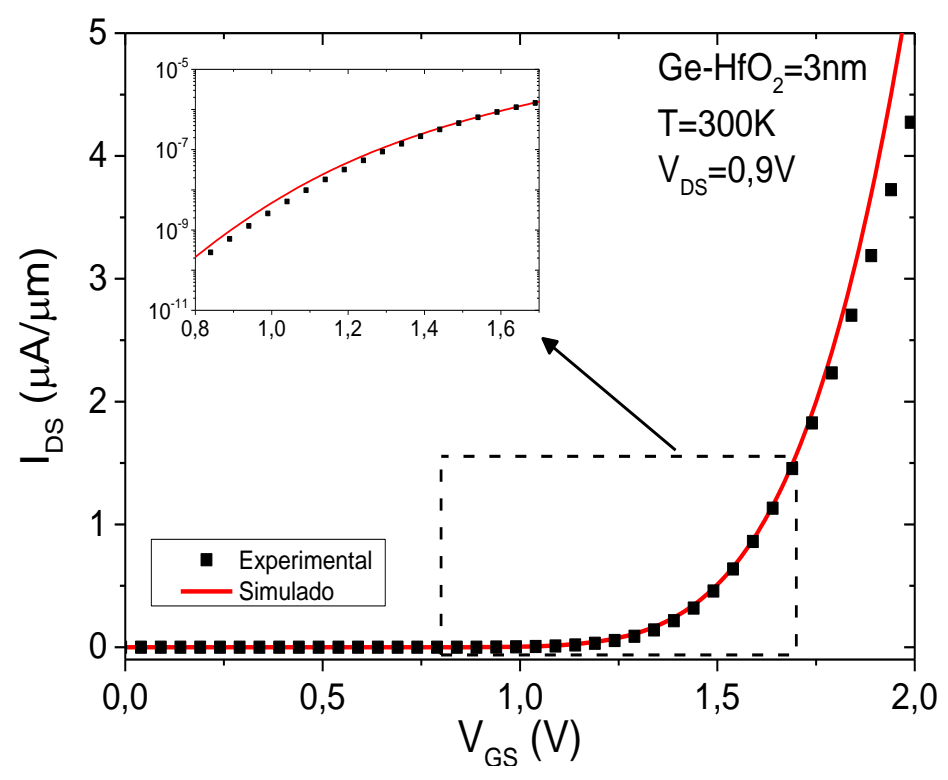

Fonte: Autor 
A partir dessas simulações, foi extraído o perfil da taxa de geração e recombinação de elétrons devido ao BTBT ao longo do dispositivo e plotado na figura 4.18. Considerando $L_{T}$ o espalhamento desses elétrons na interface fonte/canal, foi obtido $L_{T}=7,5 \mathrm{~nm}$.

Figura 4.18 - Perfil de geração e recombinação devido à BTBT obtido por simulação ao longo do dispositivo

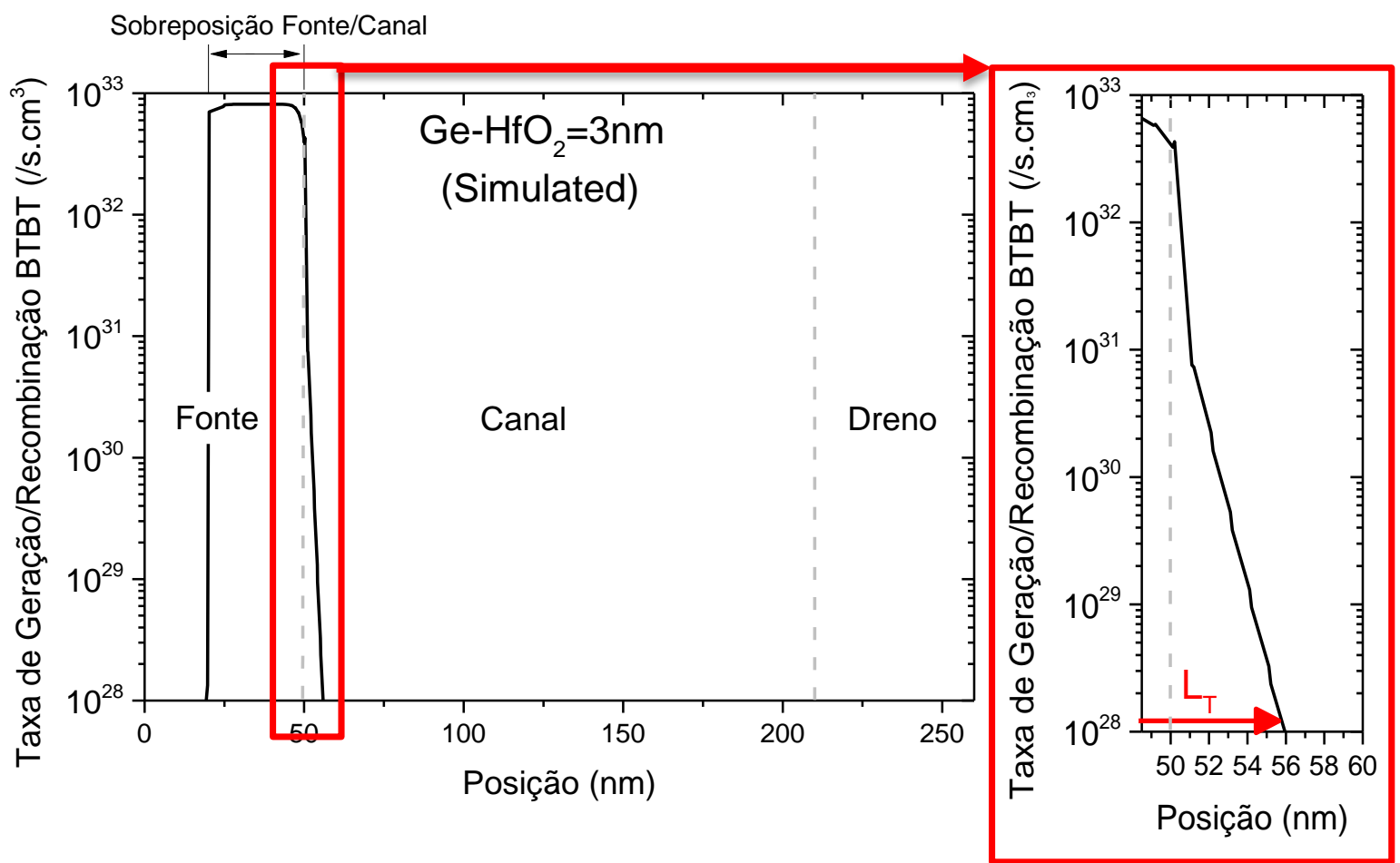

Fonte: Autor

Para a extrair o valor de Not, um transistor NW-MOSFET fabricado com um processo similar ao TFET de referência foi utilizado.

O SID em função da frequência para esse MOSFET está apresentado na figura 4.19. É possível observar que para valores de $V_{G S}$ mais baixos o espectro é composto com comportamento 1/f (ruído flicker) seguido por um ruído com comportamento constante, conhecido como ruído branco (white noise).

Conforme o esperado, a curva de SID em função de IDS para um MOSFET apresenta uma tendência de $\mathrm{IDS}^{2}$, como pode ser visto na figura 4.20. 
A partir das medidas obtidas experimentalmente e usando as equações para o MOSFET (53), foi obtido $\mathrm{N}_{\mathrm{ot}}=5.10^{20} \mathrm{eV}^{-1} \mathrm{~cm}^{-3}$.

Figura 4.19 - Curva de SID x f para um transistor NW-MOSFET

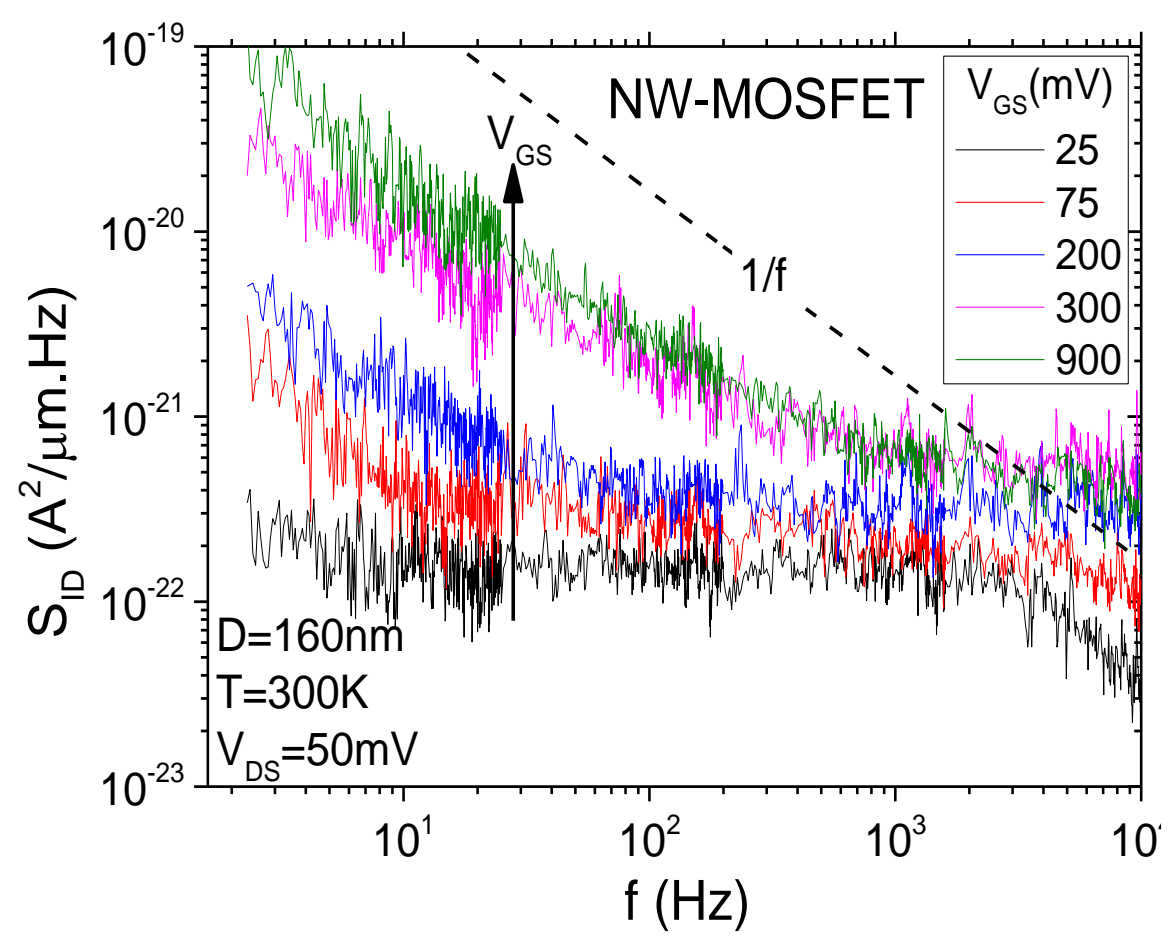

Fonte: Autor.

Figura 4.20 - Curva experimental de SID $x$ IDS para um transistor NW-MOSFET

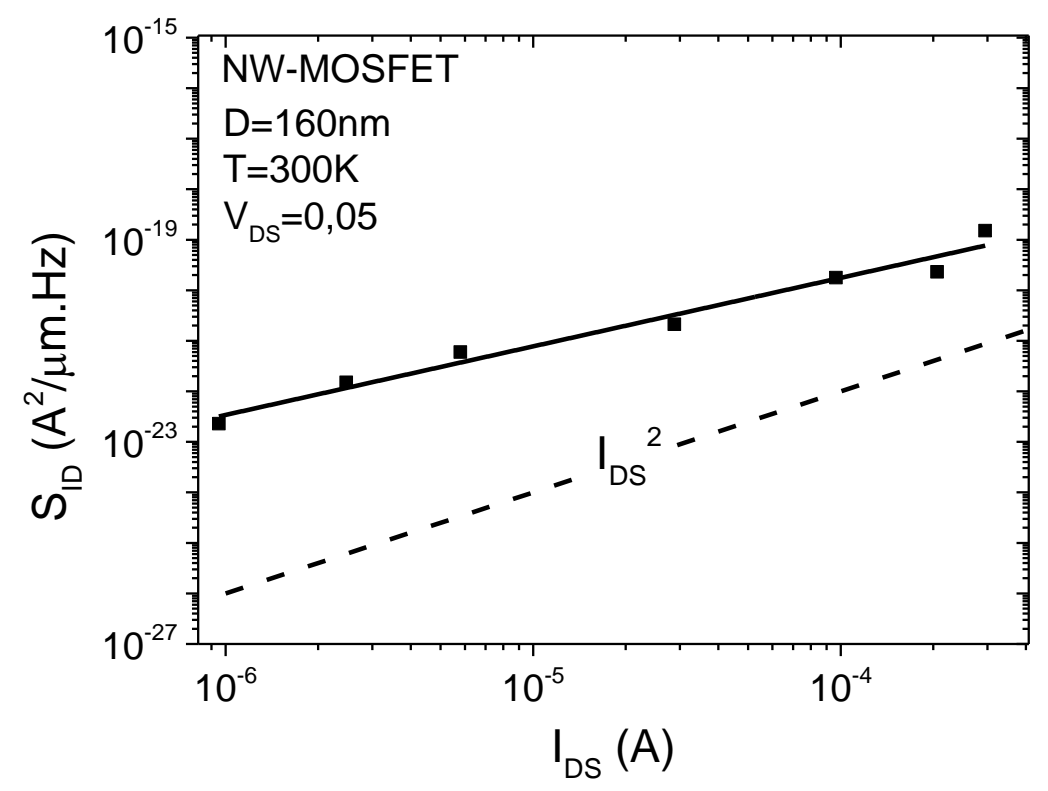

Fonte: Autor 
Finalmente, inserindo os valores de $L_{T}$ e Not extraídos na equação 4.1, - SID foi calculado e o seu resultado plotado na figura 4.21, demonstrando boa concordância com os resultados experimentais para $\mathrm{IDS}_{\mathrm{D}} \geq 10^{-7} \mathrm{~A} / \mu \mathrm{m}$. Este resultado sugere que é possível realizar um modelo de ruído em baixa frequência para TFETs usando como princípio o modelo já existente para MOSFETs.

Figura 4.21 Curvas de SID x IDs para um NW-TFET com fonte de Ge obtidas de experimentalmente e o modelo proposto

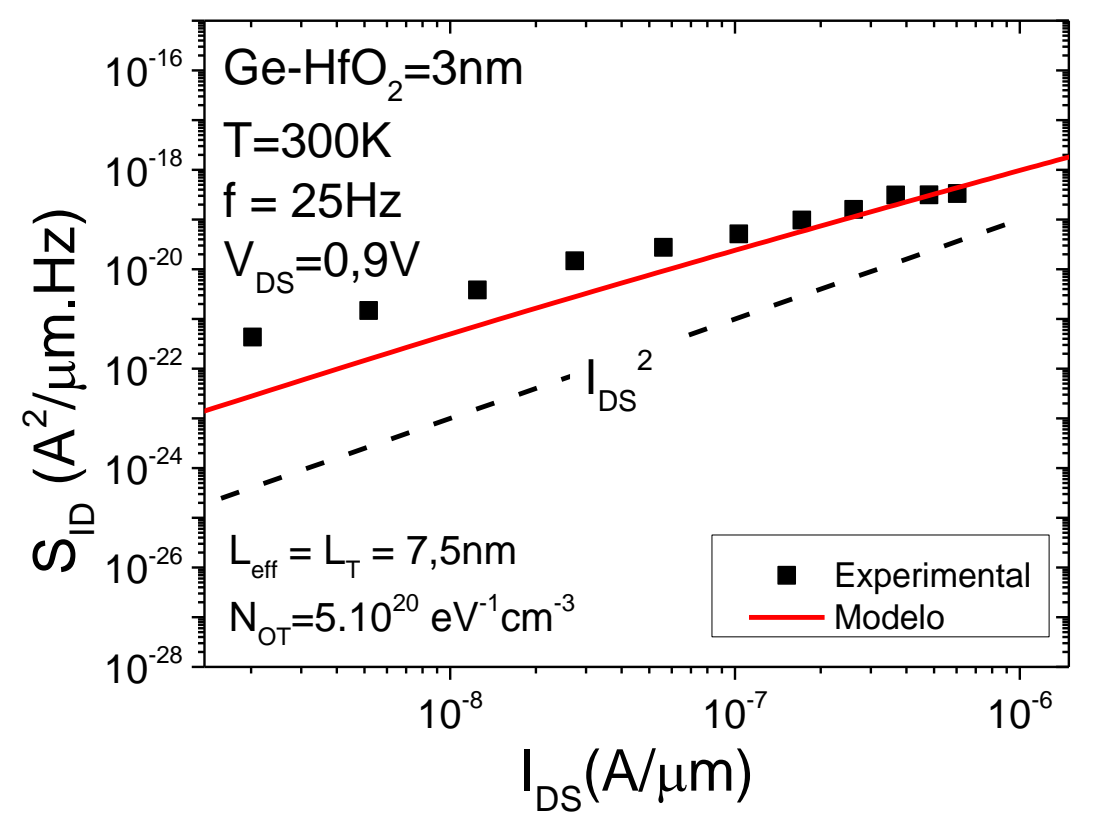

Fonte: Autor

\subsubsection{Estudo de ruído em baixa temperatura}

$\mathrm{Na}$ figura 4.22, estão plotadas as curvas de IDS em função de VGs em temperatura ambiente $(\mathrm{T}=300 \mathrm{~K})$ e $77 \mathrm{~K}$ para um TFET com fonte de $\mathrm{Ge} e$ $\mathrm{tHfO}_{\mathrm{H}}=3 \mathrm{~nm}$.

Em baixas temperaturas os mecanismos termicamente dependentes, como TAT e geração e recombinação $\mathrm{SRH}$ são minimizados, chegando a serem desprezíveis em temperaturas mais baixas. Dessa forma, ocorre o aumento da influência do BTBT para tensões mais baixas. Conforme apresentado, para este dispositivo quando $\mathrm{V}_{\mathrm{GS}}<1,3 \mathrm{~V}$ os mecanismos de condução predominante são o TAT 
e o $\mathrm{SRH}$, que apresentam uma relação direta com temperatura, resultando na redução de lDs na região de desligamento do transistor (lofF) em temperaturas mais baixas.

Porém, para $\mathrm{V}_{\mathrm{GS}}>1,3 \mathrm{~V}$, o mecanismo de condução predominante é o BTBT, que possui uma relação indireta com a temperatura através de $E_{g}$, apresentando assim, uma diminuição relativamente menor na corrente lon do dispositivo para temperaturas mais baixas.

Figura 4.22 Curva experimental de lDs $x$ VGs para um NW-TFET com T $=300 \mathrm{~K}$ e $77 \mathrm{~K}$

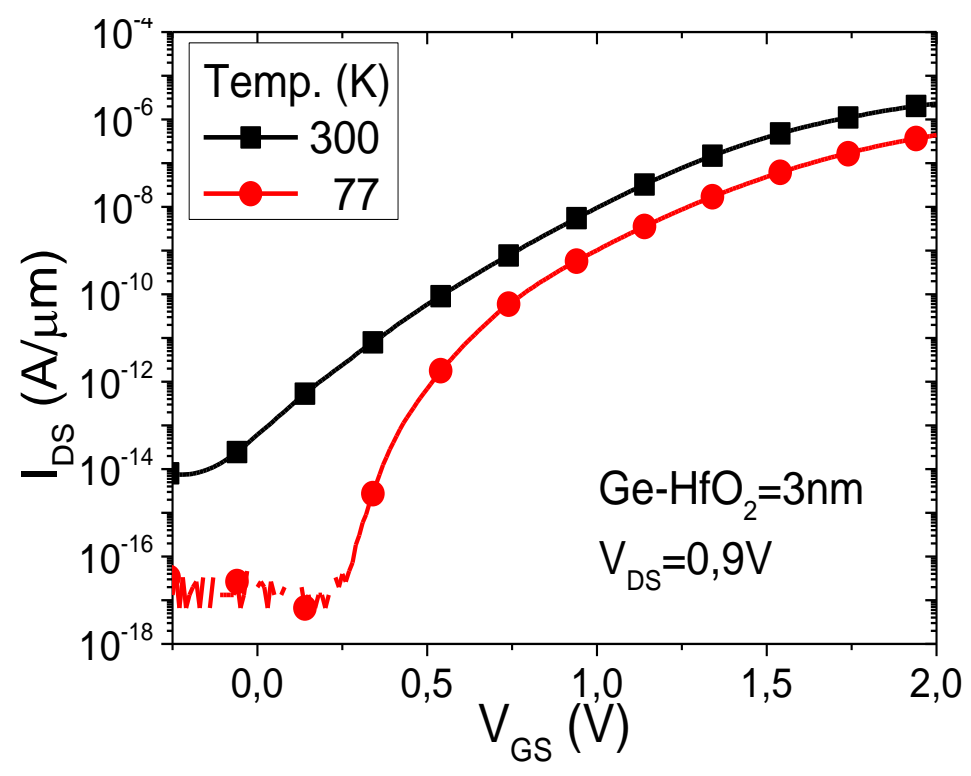

Fonte: Autor

A figura 4.23 apresenta as curvas de SID e SID normalizado por IDS ${ }^{2}$, ambas em função de IDS e com T = $77 \mathrm{~K}$ e $300 \mathrm{~K}$. A faixa de IDs apresentado nesta figura varia de $10^{-12} \mathrm{~A} / \mu \mathrm{m}$ à $10^{-6} \mathrm{~A} / \mu \mathrm{m}$, que em temperatura ambiente compreende as regiões de TAT e BTBT.

Nota-se nesta figura que nenhuma variação significativa com a redução de temperatura foi observada. Considerando que ruído em TFET deve-se ao armadilhamento e desarmadilhamento no dielétrico de porta e isso ocorre devido à presença de от, este resultado indica que a variação de Nот com a temperatura (55), não é grande o suficiente para influenciar nos níveis de ruído deste transistor. 
Figura 4.23 - Curvas de SID e SID normalizado por $\operatorname{lDS}^{2}$ para um NW-TFET com fonte de Ge

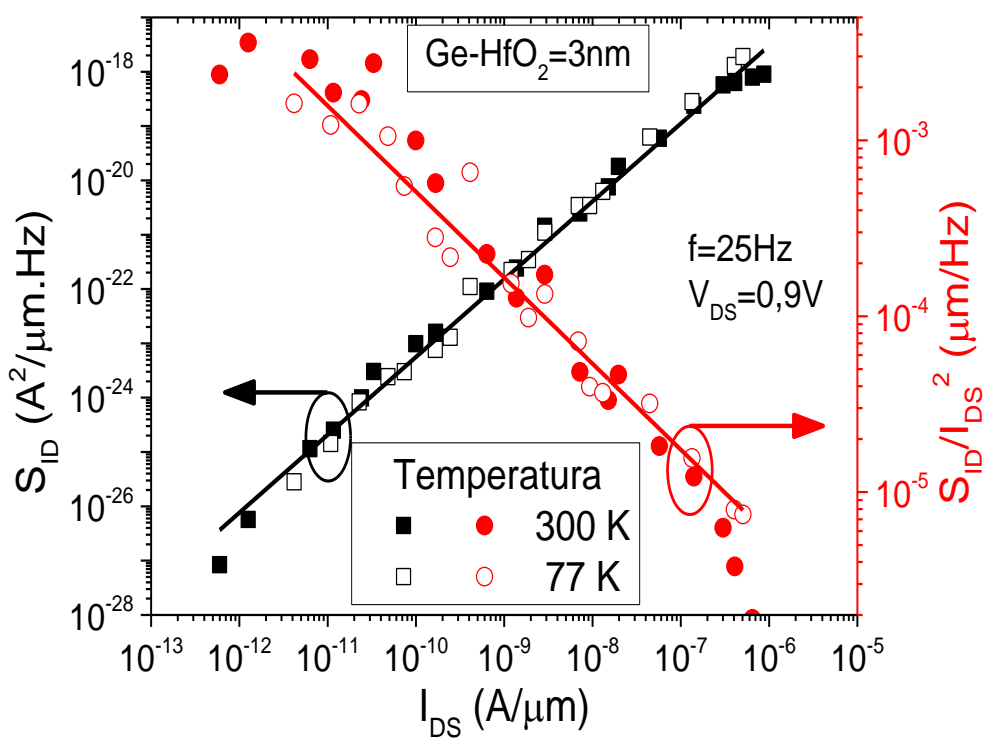

Fonte: Autor

As curvas de Svg em função de IDs para $T=77 \mathrm{~K}$ e $300 \mathrm{~K}$ estão plotadas na figura 4.24. Desta figura, é possível observar que devido a redução da contribuição do TAT na componente da corrente, o Svg é fortemente reduzido para baixos valores de IDS, onde este mecanismo de condução é predominante. Observa-se também que a região de Svg constante se entende para valores mais baixos de IDs em $T=77 \mathrm{~K}$, demonstrando o aumento da influência de BTBT nesta região, estando de acordo com a interpretação anterior.

Figura 4.24 - Curvas experimentais de Svg x lDs para um NW-TFET com fonte de Ge

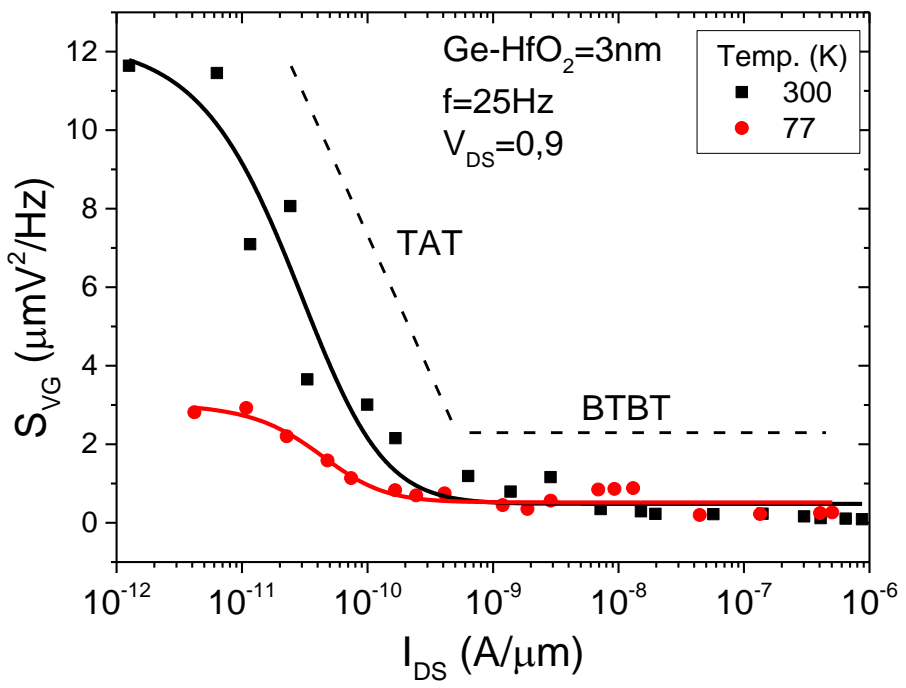

Fonte: Autor 


\subsection{Estudo de histerese em TFETs com fonte de Ge}

A densidade de armadilhas de interface $\left(\mathrm{N}_{\mathrm{it}}\right)$ e a densidade de armadilhas no óxido ( $\mathrm{N}_{\mathrm{ot}}$ ) são efeitos indesejados nos transistores TFETs e um dos impedimentos para que este dispositivo atinja valores de inclinação de sublimiar (SS) abaixo do limite teórico, por isso seu estudo torna-se necessário.

Com o intuito de verificar a influência da densidade de armadilhas no óxido (Nот) no comportamento dos TFETs, foi realizado um estudo para a verificar se estes dispositivos apresentavam comportamento de histerese. Para a realização deste estudo foram utilizados TFETs com fonte de Ge (lâminas 5 e 7), sendo eles com diferentes espessuras de $\mathrm{HfO}_{2}(3 \mathrm{~nm}$ e $2 \mathrm{~nm})$ já apresentados e com duas dimensões descritas na tabela 4.1 .

A comparação das curvas de IDS $x$ VGs em temperatura ambiente ( $T=300 \mathrm{~K}$ ) para estes dois dispositivos estão apresentadas nas figuras 4.3 e 4.8.

Para baixas temperaturas a influência do TAT e da geração e recombinação SRH são minimizados, fazendo com que o BTBT prevaleça sobre estes mecanismos de condução e assim permitindo que o TFET atinja valores ainda menores de SS.

A influência da redução de temperatura no comportamento destes transistores pode ser observada nas figuras $4.25(A)$ e $(B)$, onde estão plotadas as curvas de IDS em função de $V_{G S}$, com $T$ variando de $300 \mathrm{~K}$ a $78 \mathrm{~K}$, para os transistores com thfO2 de $3 \mathrm{~nm}$ (referência) e $2 \mathrm{~nm}$.

Considerando o gráfico de $\mathrm{E}_{\mathrm{A}} \times \mathrm{V}_{\mathrm{GS}}$, apresentado na figura 4.9, observa-se mais uma vez que para $V_{\text {Gs }}$ maior que 1,3 V a corrente de dreno é dominada por BTBT, e conforme esperado, apresentando uma pequena variação relativa de IDS devido a fraca dependência com a temperatura. Entretanto, para a região de baixo campo elétrico ( $V_{G S}<1,3 \mathrm{~V}$ ), o mecanismo predominante é o TAT, sendo este diretamente dependente da temperatura, resultando em uma redução relativamente maior de IDS para menores temperaturas. 
Figura 4.25 - Curvas experimentais de $I_{D S} \times V_{G S}$ em função da temperatura para transistores NW-TFET com espessuras de $\mathrm{HfO}_{2}$ de $3 \mathrm{~nm}(\mathrm{~A})$ e $2 \mathrm{~nm}(\mathrm{~B})$
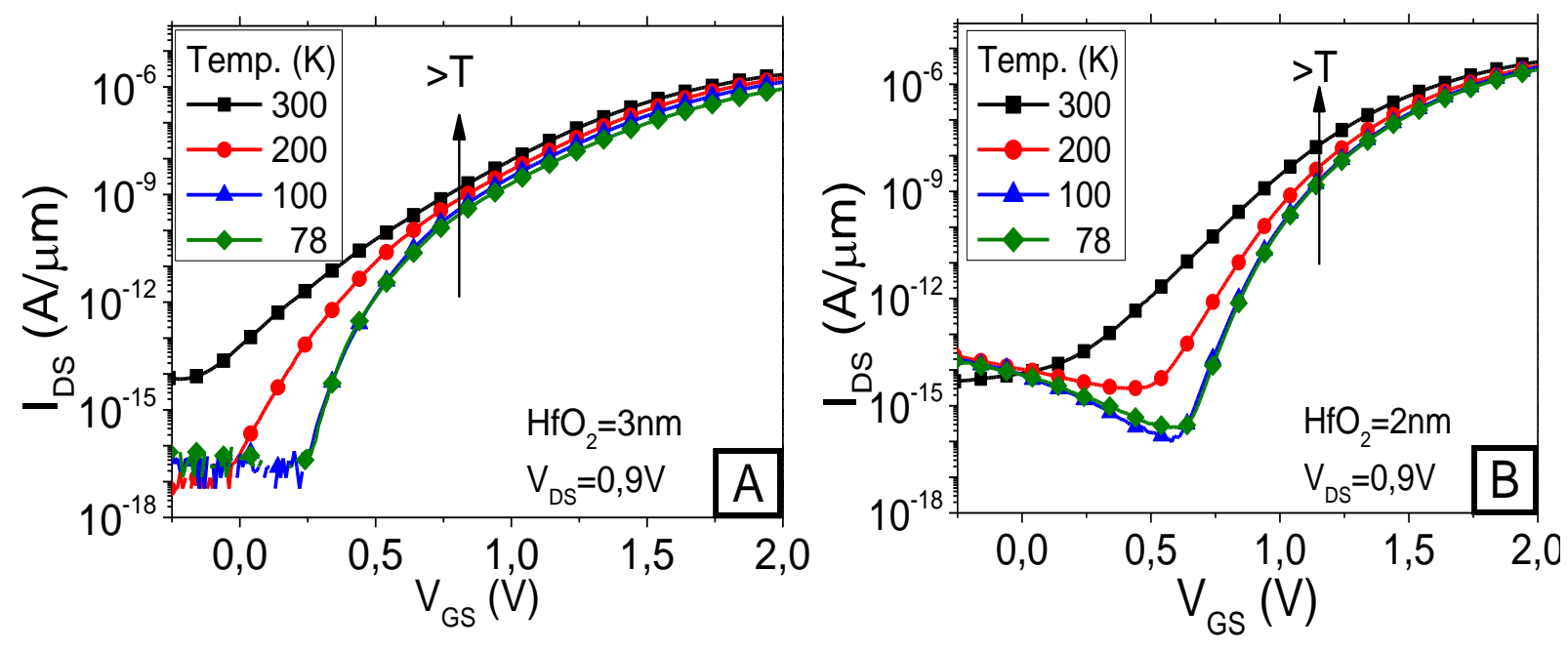

Fonte: Autor

Quando uma varredura dupla (double sweep) é realizada no TFET de referência, observa-se o fenômeno de histerese. Quando a tensão de porta é variada de forma crescente (de $-0,5 \mathrm{~V}$ à 2,0 V), as armadilhas no oxido são carregadas com elétrons e quando $V_{G s}$ é variado de forma decrescente (de 2,0 V à - $0,5 \mathrm{~V}$ ), ocorre a descarga destes elétrons armadilhados no óxido, ocasionando o deslocamento de IDS na curva, como pode ser observado na figura 4.26 (a). Entretanto, nota-se na figura 4.26 (b) que o TFET com 2 nm de $\mathrm{HfO}_{2}$ não apresentou histerese.

Figura 4.26 - Curvas experimentais com dupla varredura de IDS $\times V_{G S}$ em função da temperatura para transistores NW-TFET com espessuras de $\mathrm{HfO}_{2}$ de $3 \mathrm{~nm}(\mathrm{~A})$ e $2 \mathrm{~nm}(\mathrm{~B})$
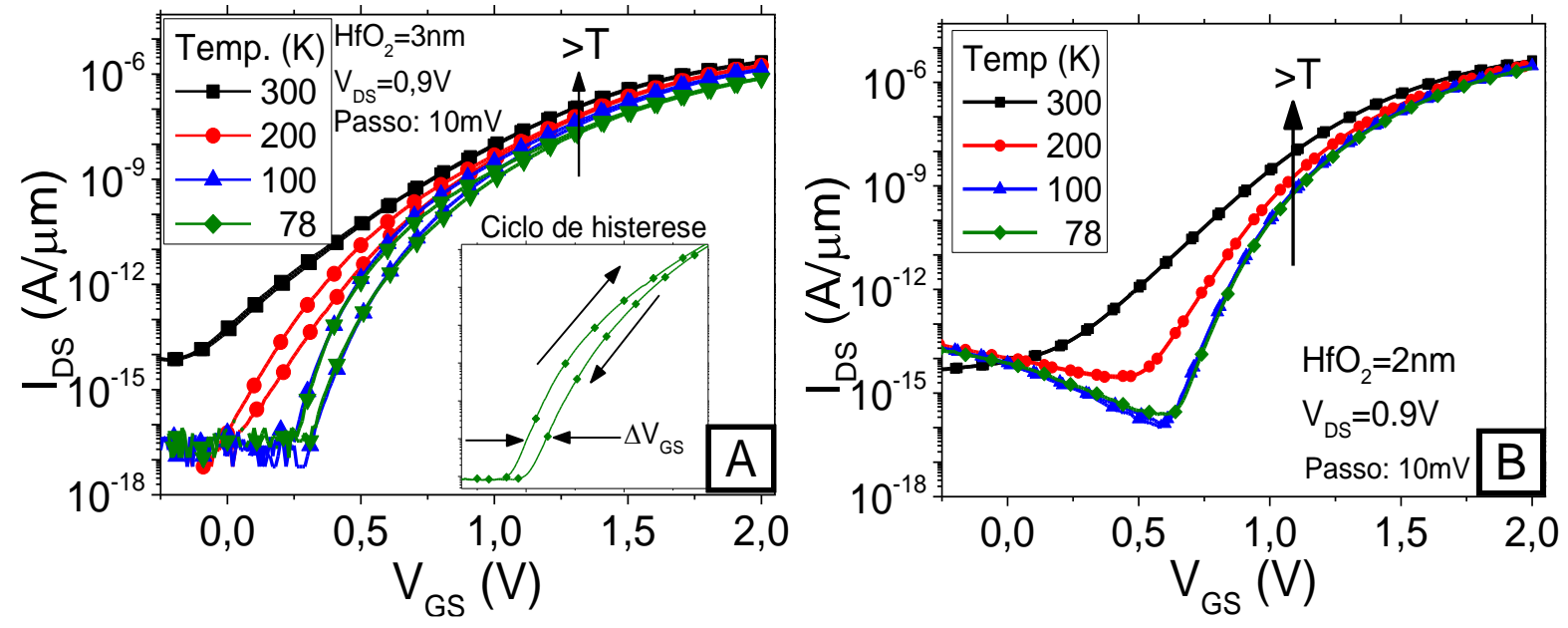

Fonte: Autor 
Os defeitos no óxido são geralmente estados de armadilhas lentas (slow-statetraps), são sensíveis à temperatura em baixo campo elétrico e sua densidade tende a aumentar conforme são resfriados (55), resultando no aumento de $\Delta V_{G s}$ em baixas temperaturas. Os valores de $\Delta \mathrm{V}_{\mathrm{GS}}$ em $\mathrm{IDS}=10^{-12} \mathrm{~A} / \mu \mathrm{m}$ obtidos para as diferentes temperaturas estão apresentados na figura 4.27 e sumarizados na tabela 4.4.

Figura 4.27 - Curva de $\Delta \mathrm{V}_{\mathrm{GS}} \times \mathrm{T}$ para o NW-TFET com $3 \mathrm{~nm}$ de $\mathrm{HfO}_{2}$

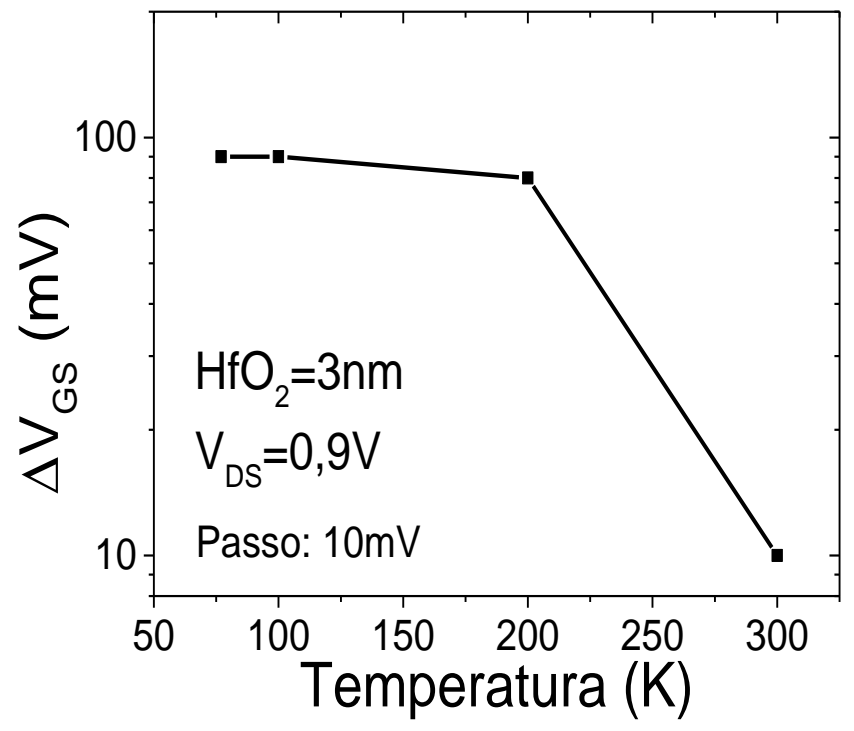

Fonte: Autor

Tabela 4-3 - Valores de $\Delta \mathrm{V}_{\text {GS }}$ e Not em diferentes temperaturas para o NW-TFET com 3nm de $\mathrm{HfO}_{2}$

\begin{tabular}{ccc}
\hline Temperatura & $\Delta V_{G S}$ & $\boldsymbol{N}_{\boldsymbol{O} \boldsymbol{T}}=\frac{\Delta \boldsymbol{V}_{\boldsymbol{G} \boldsymbol{S} \cdot \boldsymbol{\boldsymbol { C } _ { \boldsymbol { o } }}}}{\mathbf{q}}$ \\
\hline $300 \mathrm{~K}$ & $10 \mathrm{mV}$ & $1,47.10^{11} \mathrm{~cm}^{-2}$ \\
$200 \mathrm{~K}$ & $80 \mathrm{mV}$ & $1,18.10^{12} \mathrm{~cm}^{-2}$ \\
$100 \mathrm{~K}$ & $90 \mathrm{mV}$ & $1,32.10^{12} \mathrm{~cm}^{-2}$ \\
$78 \mathrm{~K}$ & $90 \mathrm{mV}$ & $1,32.10^{12} \mathrm{~cm}^{-2}$ \\
\hline
\end{tabular}

Fonte: Autor

A figura 4.28 apresenta a dependência de IDs com a variação da temperatura. Para a condição de polarização adotada, $\mathrm{V}_{\mathrm{GS}}=0,9 \mathrm{~V}$ e $\mathrm{V}_{\mathrm{DS}}=0,9 \mathrm{~V}$ e em temperatura ambiente, o mecanismo de condução predominante é o TAT. 
Com a redução de temperatura, observa-se a diminuição de IDs até que para $\mathrm{T}<100 \mathrm{~K}$, lDS atinge um platô. Nesta região de platô a influência de TAT é praticamente desprezível, fazendo com que o BTBT seja o mecanismo predominante.

Conforme pode ser observado nas figuras 4.26 (a) e melhor ressaltado na figura

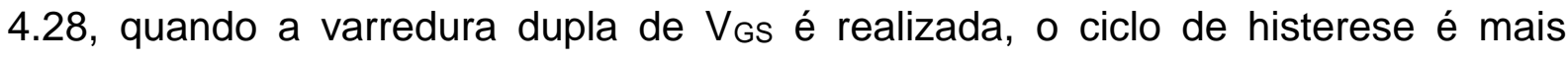
pronunciado para temperaturas mais baixas ( $T<100 \mathrm{~K}$ ), comprovando a dependência do Noт com a temperatura, sugerindo que a histerese não depende de TAT e ainda, demonstrando que não existe uma clara relação entre TAT e Not.

Figura 4.28 - Curva de lDs x 1/T para o NW-TFET com $\mathrm{HfO}_{2}$ de $3 \mathrm{~nm}$

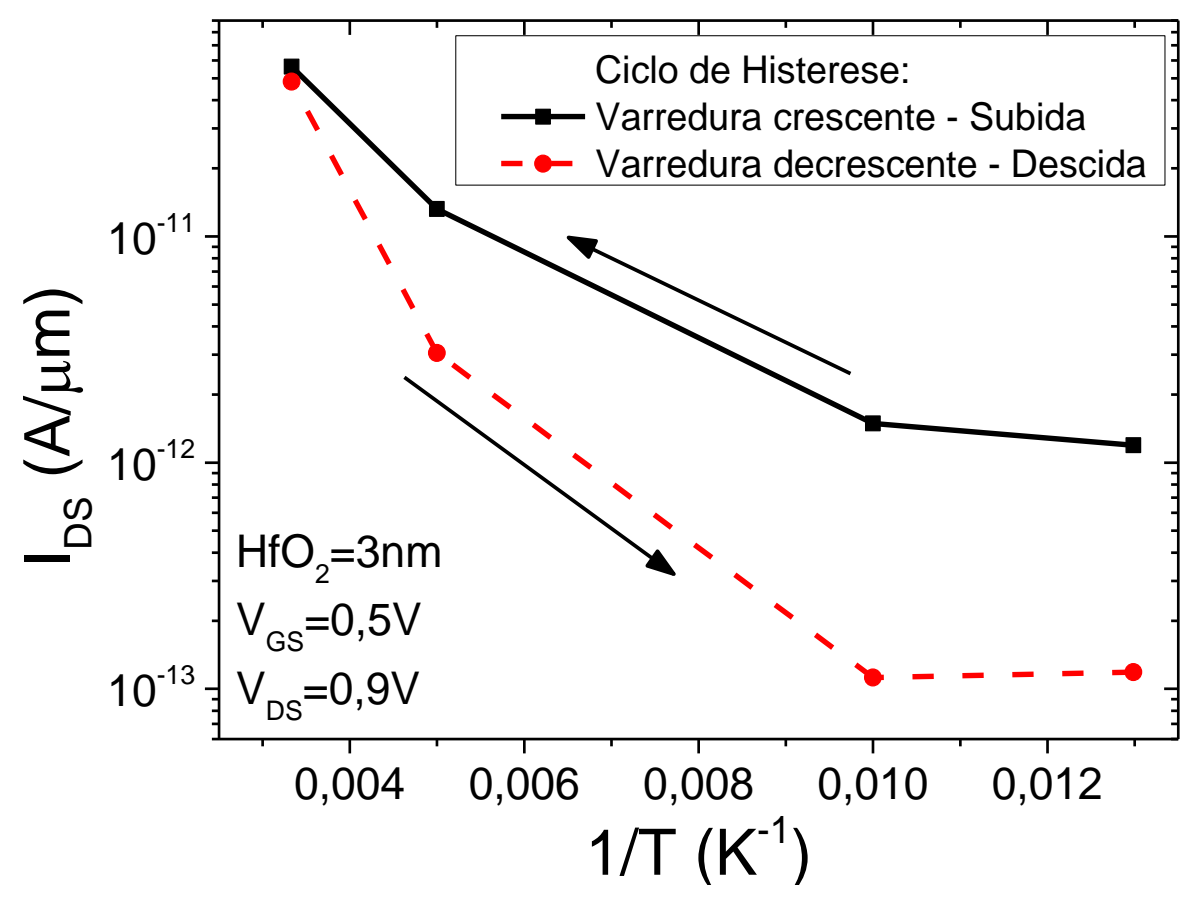

Fonte: Autor 


\section{COMPARAÇÃO DO COMPORTAMENTO ANALÓGICO DOS NW-TFETS COM FONTE DE Ge, $\mathrm{Si}_{1-\mathrm{x}} \mathrm{Ge}_{\mathrm{x}}$ e Si.}

Conforme apresentado nas seções 3 e 4, os transistores estudados neste trabalho vieram de diferentes processos de fabricação, sendo os com fonte de $\mathrm{Si}_{1-\mathrm{x}} \mathrm{Ge}_{\mathrm{x}}$ realizados em 2013 e os com fonte de Ge em 2014, onde foram realizadas modificações nas dimensões e no processo com o intuito de melhorar a performance desses transistores. A tabela 5.1 mostra essas modificações.

Apesar de todas essas diferenças nas dimensões e no processo, nesse capítulo serão reapresentadas algumas curvas do capítulo 3.5 e acrescentas as curvas do transistor com fonte de germânio (lâmina 5). Todas as discussões acerca da influência da concentração de Ge na liga $\mathrm{Si}_{1-x} \mathrm{Ge}_{x}$ dos transistores NW-TFET em função da temperatura, já foram realizadas neste mesmo capítulo (3.5).

Tabela 5-1: Dimensões dos NW-TFET provenientes de diferentes processos de fabricação

\begin{tabular}{|c|c|c|c|c|}
\hline & Lâmina 5 & Lâmina 2 & Lâmina 3 & Lâmina 4 \\
\hline Material da fonte & $\mathrm{Ge}$ & $\mathrm{Si}_{0,73} \mathrm{Ge}_{0,27}$ & $\mathrm{Si}_{0,54} \mathrm{Ge}_{0,46}$ & $\mathrm{Si}$ \\
\hline Comprimento de canal ( $\left.\mathrm{L}_{\mathrm{CH}}\right)$ & $220 \mathrm{~nm}$ & \multicolumn{3}{|c|}{$160 \mathrm{~nm}$} \\
\hline Comprimento de porta $\left(L_{G}\right)$ & $250 \mathrm{~nm}$ & \multicolumn{3}{|c|}{$150 \mathrm{~nm}$} \\
\hline Sobreposição da porta/fonte (LGS) & $85 \mathrm{~nm}$ & \multicolumn{3}{|c|}{$30 \mathrm{~nm}$} \\
\hline Subposição de porta/dreno (LGD) & $50 \mathrm{~nm}$ & \multicolumn{3}{|c|}{$40 \mathrm{~nm}$} \\
\hline Dopagem de fonte & $1.10^{20} \mathrm{~cm}^{-3}$ & \multicolumn{3}{|c|}{$1.10^{20} \mathrm{~cm}^{-3}$} \\
\hline Dopagem de canal & $1.10^{16} \mathrm{~cm}^{-3}$ & \multicolumn{3}{|c|}{$1.10^{15} \mathrm{~cm}^{-3}$} \\
\hline Dopagem de dreno & $2.10^{19} \mathrm{~cm}^{-3}$ & \multicolumn{3}{|c|}{$1.10^{20} \mathrm{~cm}^{-3}$} \\
\hline Espessura de $\mathrm{HfO}_{2}\left(\mathrm{t}_{\mathrm{HfO}}\right)$ & $3 \mathrm{~nm}$ & \multicolumn{3}{|c|}{$3 \mathrm{~nm}$} \\
\hline Diâmetro (D) & $200 \mathrm{~nm}$ & \multicolumn{3}{|c|}{$200 \mathrm{~nm}$} \\
\hline
\end{tabular}

Fonte: Autor

A figuras 5.1, apresenta as curvas de IDS $\times V_{G S}$ e IDS $\times V_{D S}$ para os transistores com diferentes composições de fonte.

Conforme já discutido, é possível observar o maior nível de lds para valores mais altos de $V_{G S}$ (figura 5.1 (A)) e VDS (figura 5.1(B)) para os transistores com fonte puramente de Ge. Isso deve-se à diminuição da banda de energia proibida $\left(E_{g}\right)$, 
resultando no aumento da corrente de tunelamento. Além disso, o transistor com fonte de $\mathrm{Ge}$ apresenta maior sobreposição de porta e um perfil de junção fonte/canal aproximadamente abrupto, o que também contribui para valores mais altos de corrente.

Figura 5.1: Curvas experimentais de lDS $x V_{G S}(A)$ e lDS $x V_{D S}(B)$ para transistores NW-TFET com diferentes composições de fonte
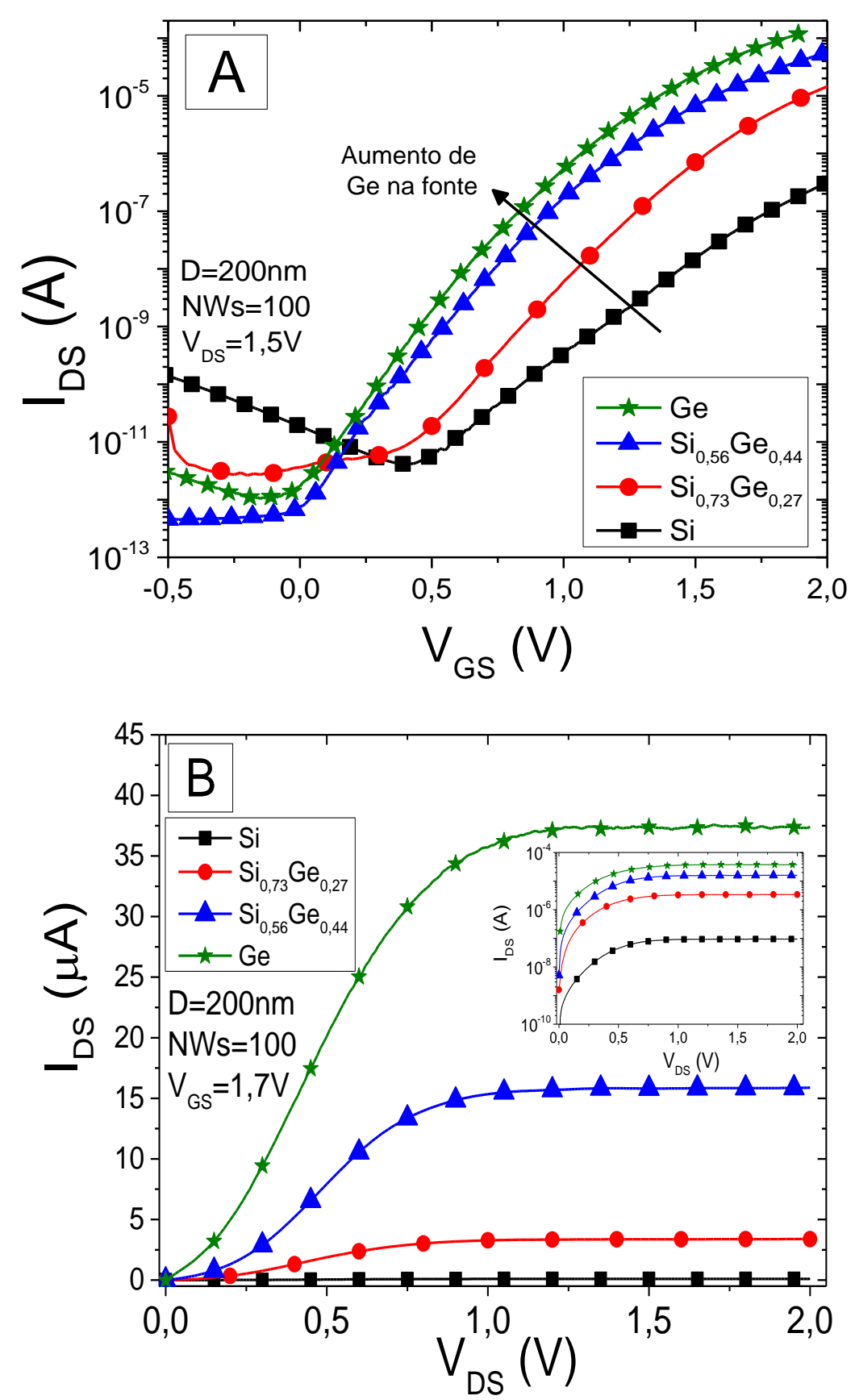

Fonte: Autor 
O aumento da corrente lon, sem piorar a degradação de loff, faz com que o transistor com fonte de $\mathrm{Ge}$ apresente uma melhor relação lon/loff e consequentemente menor inclinação de sublimiar (SS).

A partir da figura 5.1(B), nota-se mais uma vez que todos os dispositivos apresentam uma região de IDs continuo, muito similar a região de saturação de um transistor MOSFET, podendo ser relacionado à saturação de tunelamento.

A figura 5.2 apresenta as curvas de $g_{m}(A)$ e $g_{d}(B)$ em função da porcentagem de $\mathrm{Ge}$ na fonte. Considerando $\mathrm{Si}_{1-\mathrm{x}} \mathrm{G}_{\mathrm{x}}$, quando $\mathrm{x}=0$ obtemos $0 \%$ de $\mathrm{Ge}$, representando apenas $\mathrm{Si}$ e quando $\mathrm{x}=1$, obtemos $100 \%$ de Ge.

Figura 5.2: Curvas experimentais de $g_{m}(A)$ e $g_{d}(B)$ em função da porcentagem de Ge na fonte
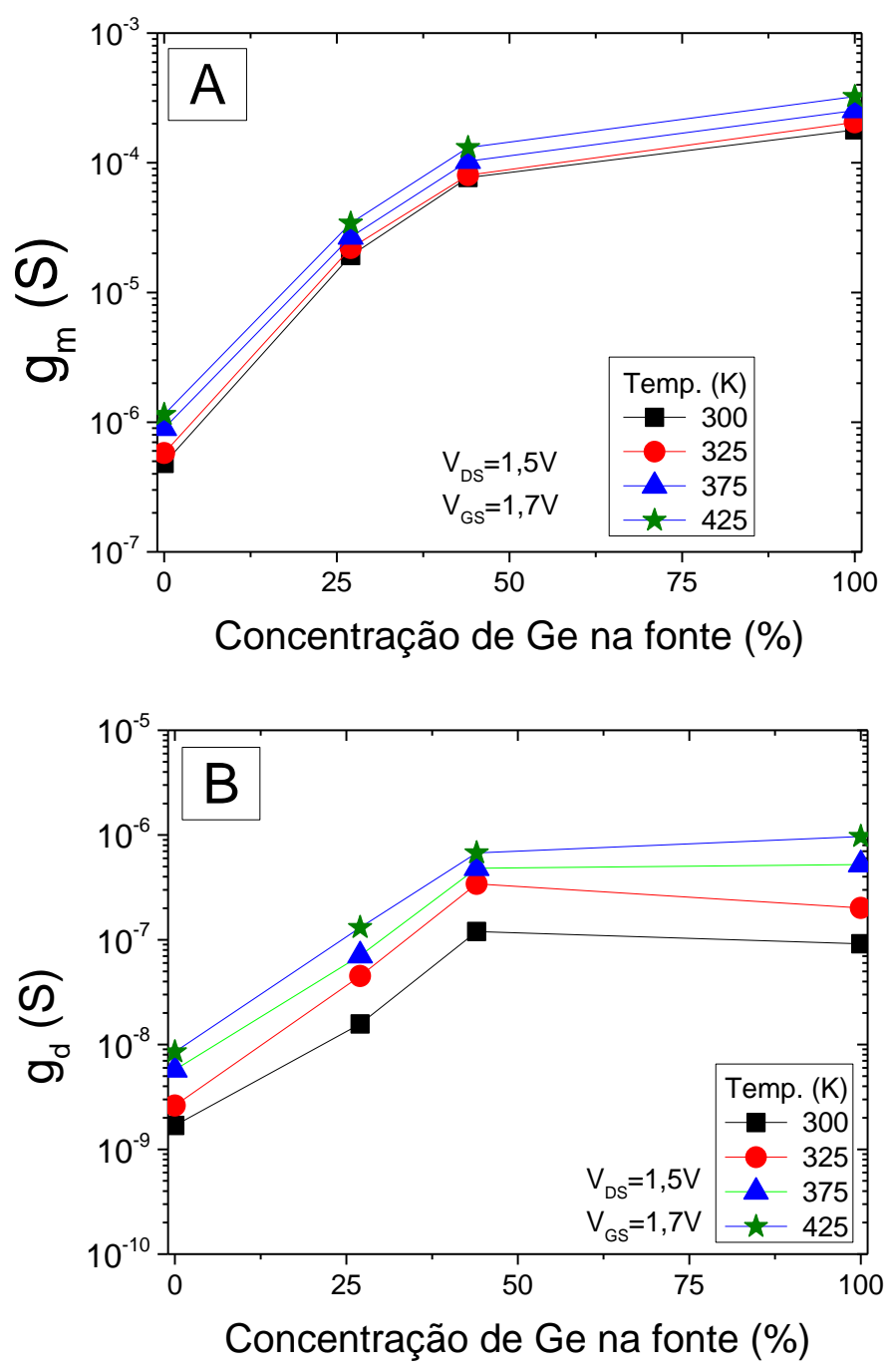

Fonte: Autor 
Conforme o esperado e já discutido, é possível observar na figura 5.2(A) o aumento de $\mathrm{gm}_{\mathrm{m}}$ para os transistores com fonte puramente de Ge, quando comparados aos outros dispositivos. Isto ocorre devido ao aumento da corrente de tunelamento direto de banda para banda (BTBT). Além disso, nota-se a baixa influência da temperatura em todos os dispositivos, induzindo apenas um pequeno aumento relativo de $g_{m}$ devido a dependência indireta de BTBT com a temperatura através de $E_{g}$.

A partir da figura 5.2 (B) nota-se a degradação da condutância de saída com o aumento de $\mathrm{Ge}$, isso ocorre devido a dependência de BTBT com o campo elétrico. Ou seja, altos valores de $V_{D S}$ induzem altos valores de campo elétrico, resultando em maiores degradações de $g_{d}$. Além disso, é possível observar que gd tende a saturar para $x=0,5$ ( $50 \%$ de $\mathrm{Ge}$ ). Quando observado a influência da temperatura, todos os dispositivos tendem a exibir um aumento muito similar com a variação positiva de temperatura.

E por fim, a figura 5.3 apresenta a curva do ganho intrínseco de tensão (Av) em função da porcentagem de Ge na fonte.

Figura 5.3: Curvas experimentais de Av em função da porcentagem de Ge na fonte

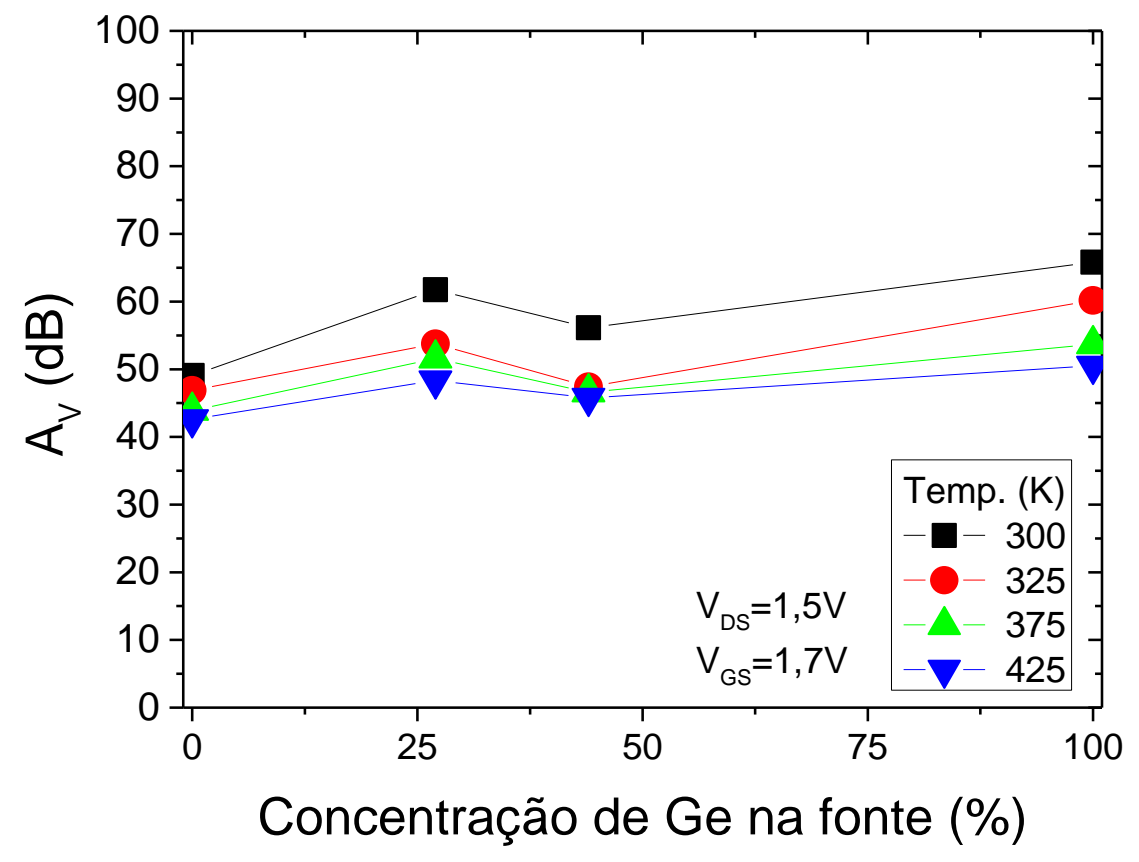

Fonte: Autor 
Desta figura, nota-se uma pequena melhora no ganho Av dos transistores com fonte de Ge em relação aos com fonte de Si. Essa variação de AV decorre da competição entre o aumento de $g_{m}$ e a degradação de $g_{d}$.

Conforme apresentado no capitulo 3.5, os dispositivos com fonte de $\mathrm{Ge}$ apresentam melhores ganhos de Av quando comparado ao com fonte de Si devido à melhora no $g_{m}$. Porém, à maior degradação de $g_{d}$, resulta em piores valores de $A_{v}$ para o NW-TFET com $46 \%$ de Ge quando comparado ao com $27 \%$ de Ge.

Para o dispositivo com fonte de Ge (100\%), quando comparado aos outros dispositivos, ocorre a melhora do $A_{v}$ devido ao maior aumento relativo de $\mathrm{gm}_{\mathrm{m}} \mathrm{em}$ relação ao aumento de $g_{d}$,

Quando analisado a influência da temperatura, observa-se a degradação de Av com o aumento da temperatura. E essa influência é ainda maior para os dispositivos com fonte de $\mathrm{Ge}$, apresentando maior redução no valor de $\mathrm{Av}$, devido à maior degradação de gd com a temperatura. 


\section{CONCLUSÕES E PROPOSTAS DE TRABALHOS FUTUROS}

\subsection{Conclusões}

Com base em simulações numéricas realizadas, foi possível observar e entender a influência dos principais mecanismos de transporte de portadores em diversas faixas de polarização dos dispositivos NW-TFET.

A partir dos diagramas de banda de energia, verificou-se que a corrente de desligamento (loff) deve-se principalmente ao mecanismo de geração e recombinação SRH. E quando considerado um nTFET, para tensão de porta positiva ocorre o aparecimento de duas regiões, uma intermediaria dominada pelo mecanismo de tunelamento induzido por armadilhas (TAT) e para $\mathrm{V}_{\text {GS }}$ mais altos, acima de 1,0 V, outra predominada pelo tunelamento direto de banda para banda (BTBT).

Conhecendo-se da literatura a dependência (forte ou fraca) de cada um desses mecanismos com a temperatura e levando em conta o observado através das simulações, foi possível verificar experimentalmente a influência da temperatura no comportamento do NW-TFET.

Constatou-se que a geração e recombinação SRH é diretamente dependente da temperatura, resultando na degradação da corrente de desligamento (loFF) do dispositivo com o aumento da temperatura. Além disso, o SRH ocorre devido aos elétrons armadilhados em níveis energéticos intermediários da banda proibida, portanto, foi observado que um pequeno aumento na densidade de armadilhas de interface também causando a degradação de lofF, sendo estas degradações somadas quando analisado os dois efeitos simultaneamente. Para um NW-TFET com fonte de $\mathrm{Si}_{0,73} \mathrm{Ge}_{0,27}$ e $200 \mathrm{~nm}$ de diâmetro, a relação lon/loff foi de $2.10^{6}$ em temperatura ambiente $(T=300)$ para $5.10^{3} \mathrm{em} 420 \mathrm{~K}$.

Assim como o SRH, o tunelamento induzido por armadinhas (TAT) também é diretamente dependente da temperatura e da densidade de armadilhas de interface, sendo observado que um pequeno aumento de ambos, resulta no aumento da corrente de dreno para baixas tensões de porta, além do aparecimento de uma região de platô devido ao aumento de $\mathrm{N}_{\mathrm{it}}$. 
Foi possível determinar a polarização de porta em que a corrente de tunelamento direto de banda para banda (BTBT) é predominante em cada um dos dispositivos. Para isso foram utilizadas medidas experimentais em função da temperatura, e calculado através do método de Arrheinius. Esta região dominada por BTBT demonstrou-se pouco dependente com $\mathrm{T}$ e Nit. Porém demonstrou-se ser bastante dependente do estreitamento da banda proibida $\left(E_{g}\right)$. Para um transistor NWTFET com fonte de Ge e SiGe, o mecanismo de condução BTBT é predominante para VGs maiores que $1,3 \mathrm{~V}$, enquanto os NW-TFET com fonte de Si apresentam essa predominância para valores de $V_{G S}$ próximo ou maior que 2,0V.

$O$ uso de diferentes materiais de fonte com menor $E_{g}$, ou seja, maior porcentagem de Ge, resultou no aumento da corrente lon em até 4 ordens de grandeza quando comparado dispositivos com fonte de puramente de Ge e Si. Em contrapartida, essa substituição pode resultar no aumento de imperfeições, resultando no aumento da densidade de armadilhas de interface ( $\mathrm{N}_{\mathrm{it}}$ ), piorando a corrente loff.

Observou-se que apesar dessa degradação de loff, os transistores com fonte de Ge ou SiGe ainda assim, apresentaram melhor relação lon/loff e menores valores de SS quando comparados aos com fonte de Si.

Com isso concluiu-se que devido à degradação da corrente loff com a temperatura, resultou na degradação da relação lon/loff e da inclinação de sublimiar, fazendo com que este dispositivo seja pouco interessante para aplicações digitais em altas temperaturas. Porém apresentou um comportamento muito constante para altos valores de tensão de dreno e devido à baixa influência da temperatura na corrente de tunelamento, demonstrando potencial para aplicações analógicas.

Foram obtidos experimentalmente as principais figuras de mérito para análise dos parâmetros analógicos, como (transcondutância) $g_{\mathrm{m}}$, condutância de saída $\left(\mathrm{gd}_{\mathrm{d}}\right.$ e ganho intrínseco de tensão $(A v)$. Para garantir que os transistores estivessem operando na região onde o BTBT é predominante, foram utilizados VDS e $V_{G S}$ acima de $1 \mathrm{~V}$.

O aumento da corrente de tunelamento propiciado pelo uso de Ge na fonte resultou no aumento de $\mathrm{gm}_{\mathrm{m}}$ em até 3 décadas quando comparado à um dispositivo com fonte de Si. Além disso, foi observado a baixa influência da temperatura, onde 
para altas temperaturas foi notado apenas um pequeno aumento relativo, devido a dependência indireta de BTBT com a temperatura através de $E_{g}$. Entretanto, BTBT é fortemente dependente do campo elétrico, sendo observado o aumento de $\mathrm{g}_{\mathrm{d}}$, ou seja, piora, para tensões mais altas de $V_{D S}$.

Sendo o ganho intrínseco de tensão proveniente da relação $\mathrm{g}_{\mathrm{m}} / \mathrm{g}_{\mathrm{d}}$, notou-se que a predominância do aumento de $\mathrm{gm}_{\mathrm{m}}$, resultando em melhores resultados para os NWTFET com maior porcentagem de Ge na fonte, $\mathrm{Av}=66 \mathrm{~dB}$ em temperatura ambiente $(\mathrm{T}=300 \mathrm{~K})$ e $\mathrm{A}_{v}=50 \mathrm{~dB}$ para $\mathrm{T}=425 \mathrm{~K}$, enquanto o NW-TFET com fonte de Si apresentou $A v=49 \mathrm{~dB}$ para $\mathrm{T}=300 \mathrm{~K}$ e $42 \mathrm{~dB}$ para $\mathrm{T}=425 \mathrm{~K}$.

Além do aumento das armadilhas de interface, o uso de diferentes materiais pode gerar defeitos no óxido, como introdução de impurezas. Para estudar a influência dessas impurezas no comportamento dos transistores, foi realizado o estudo de ruído em baixa frequência.

Para os TFETs os ruídos em baixa frequência ocorrem devido ao armadilhamento e desarmadilhamento de elétrons no óxido. Os transistores com fonte de $\mathrm{Ge}$ e $3 \mathrm{~nm}$ de $\mathrm{HfO}_{2}$ no dielétrico de porta, apresentaram níveis de ruído flicker uma década maior quando comparado ao puramente de $\mathrm{Si}$.

Entretanto, a redução da espessura para $2 \mathrm{~nm}$ de $\mathrm{HfO}_{2}$ no dielétrico de porta, demonstrou reduzir a densidade de armadilhas no oxido, resultando na redução de aproximadamente 2 décadas nos níveis de ruído flicker, quando comparado ao dispositivo com $3 \mathrm{~nm}$ de $\mathrm{HfO}_{2}$. Além disso, o uso de espessura reduzida de $\mathrm{HfO}_{2}$ resultou no melhor acoplamento eletrostático do dispositivo, menor degradação de loFF, aumento da região onde BTBT é predominante. E assim, resultando em melhor relação lon/loff, menores valores de SS, menor degradação de gd e maior ganho Av.

Neste trabalho foi proposto o uso do modelo de ruído em baixa frequência do MOSFET para TFET, fazendo-se apenas algumas alterações para manter a compatibilidade, como a troca do comprimento efetivo de canal (Leff) pelo comprimento de tunelamento $\left(L_{T}\right)$. Concluiu-se que este modelo pode ser usado como base para um futuro modelo de TFET. 


\subsection{Propostas de trabalhos futuros}

Como proposta de trabalhos futuros existe a possibilidade de realizar a caraterização elétrica de dispositivos com novos materiais de fonte como III-V, que até o presente momento encontra-se em processo de fabricação no Imec, Bélgica.

Durante o estudo de ruído flicker em baixa frequência, também foi observado a presença de ruído RTN (Randon Telegraph Noise), abrindo margem para o estudo desse tipo de ruído no domínio do tempo.

Além disso, o modelamento de ruído apresentado neste trabalho foi um estudo inicial. Este modelo pode ser aprofundado e a partir dos resultados aqui apresentados, gerar um modelo especifico para TFETs. 


\section{PUBLICAÇÕES GERADAS DURANTE O DOUTORADO}

\section{Publicações em revistas}

AGOPIAN, P. G. D.; MARTINO, M. D. V.; SANTOS, S. D.; NEVES, F. S.; MARTINO, J. A; ROOYACKERS, R.; VANDOOREN, A.; SIMOEN, E.; THEAN, A.; CLAEYS, C. Influence of the Source Composition on the Analog Performance Parameters of Vertical Nanowire-TFETs. IEEE Transactions on Electron Devices, v. 62 , n. 1, p. 16-22, 2015.

MARTINO, M. D.; NEVES, F. S; AGOPIAN, P. G. D.; MARTINO, J. A.; ROOYACKERS, R.; VANDOOREN, A.; SIMOEN, E.; THEAN, A.; CLAEYS, C. Analog Performance of Vertical Nanowire TFETs as a Function of Temperature and Transport Mechanism. Solid State Electronics, v. 112, p. 51-55, 2015.

MARTINO, M. D.; NEVES, F. S; AGOPIAN, P. G. D.; MARTINO, J. A.; ROOYACKERS, R.; VANDOOREN, A.; SIMOEN, E.; THEAN, A.; CLAEYS, C. Nanowire Tunnel Field Effect Transistors at High Temperature. JICS. Journal of Integrated Circuits and Systems, v. 8, p. 110-115, 2013.

\section{Publicações em congressos}

NEVES, F. S.; AGOPIAN, P. G. D.; MARTINO, J. A.; ROOYACKERS, R.; VANDOOREN, A.; SIMOEN, E.; THEAN, A.; CLAEYS, C. Study of Hysteresis in Vertical Ge-Source Heterojunction Tunnel-FETs at Low Temperature. IEEE SOI-3DSubthreshold Microelectronics Technology Unified Conference, 2015, Berkeley. 
NEVES, F. S.; AGOPIAN, P. G. D.; MARTINO, J. A.; ROOYACKERS, R.; VANDOOREN, A.; SIMOEN, E.; THEAN, A., CLAEYS, C. Study of Hysteresis in Vertical Ge-Source Heterojunction Tunnel-FETs at Low Temperature. In: 227th ECS Meeting - Advanced Semiconductor-On-Insulator Technology and Related Physics, Illinois, Chicago, v. 66, p 179-185, 2015.

MARTINO, J. A.; AGOPIAN, P. G. D.; NEVES, F. S.; ROOYACKERS, R.; VANDOOREN, A.; SIMOEN, E.; THEAN, A.; CLAEYS, C. The Impact of the Ge Concentration in the Source for Vertical Tunnel-FETs. In: 227th ECS Meeting Advanced Semiconductor-On-Insulator Technology and Related Physics, Illinois, Chicago, v. 66, p. 79-86, 2015.

NEVES, F. S.; AGOPIAN, P. G. D.; MARTINO, J. A.; ROOYACKERS, R.; VANDOOREN, A.; SIMOEN, E.; THEAN, A.; CLAEYS, C. Study of Low Frequency Noise in Vertical NW-Tunnel FETs with Different Source Compositions. In: Joint International EUROSOI Workshop and International Conference on...EUROSOI-ULIS 2015, Bologna, v. 1, p. 149-152, 2015.

NEVES, F. S.; AGOPIAN, P. G. D.; MARTINO, J. A.; CRETU, B., ROOYACKERS, R.; VANDOOREN, A.; SIMOEN, E.; THEAN, A., CLAEYS, C. Influence of the gate oxide thickness on the Analog Performance of vertical NWTFETs with Ge Source. In: 10th Workshop of the Thematic Network on Silicon on Insulator Technology, Devices and Circuits - EUROSOI, 2014, Tarragona. Proceedings..., v. 1, p. 1-2., 2014. 
MARTINO, M. D.; NEVES, F. S.; AGOPIAN, P. G. D.; MARTINO, J. A.; ROOYACKERS, R.; VANDOOREN, A.; SIMOEN, E.; THEAN, A.; CLAEYS, C. Transport mechanism influence on Vertical Nanowire-TFET analog performance as a function of temperature. In: 10th Workshop of the Thematic Network on Silicon on Insulator Technology, Devices and Circuits - EUROSOI 2014, Tarragona. Proceedings..., v. 1, p. 1-2, 2014.

MARTINO, M. D.; NEVES, F. S.; AGOPIAN, P. G. D.; MARTINO, J. A.; ROOYACKERS, R.; VANDOOREN, A.; SIMOEN, E.; THEAN, A.; CLAEYS, C. Early Voltage and Intrinsic Voltage Gain in Vertical Nanowire-TFETs as a function of Temperature. In: Ninth International Caribbean Conference on DEVICES, CIRCUITS and SYSTEMS - ICCDCS 2014, Playa Del Carmem. Proceedings ... v. 1, p. 78-81, 2014.

NEVES, F. S.; AGOPIAN, P. G. D.; MARTINO, J. A.; ROOYACKERS, R.; VANDOOREN, A.; SIMOEN, E.; CLAEYS, C. Influence of interface trap density on vertical NW-TFETs with different source composition. In: Ninth Workshop of the Thematic Network on Silicon on Insulator Technology, Devices and Circuits EurosOI 2013, Paris. Proceedings... v. 1, p. 1-2, 2013.

AGOPIAN, P. G. D.; NEVES, F. S.; MARTINO, J. A.; ROOYACKERS, R.; VANDOOREN, A.; SIMOEN, E.; CLAEYS, C. Back bias influence on analog performance of PTFET. In: IEEE SOI-3D-Subthreshold Microelectronics Technology Unified Conference, 2013, Monterey. Proceedings... v. 1, p. 1-2, 2013.

AGOPIAN, P. G. D.; SANTOS, S. D.; NEVES, F. S.; MARTINO, J. A.; ROOYACKERS, R.; VANDOOREN, A.; SIMOEN, E.; CLAEYS, C. NW-TFET analog performance for different Ge source compositions. In: IEEE SOI-3D-Subthreshold Microelectronics Technology Unified Conference, 2013, Monterey. Proceedings... v. 1, p. 1-2, 2013. 
NEVES, F. S.; MARTINO, M. D. V.; AGOPIAN, P. G. D.; MARTINO, J. A; ROOYACKERS, R.; LEONELLI, D.; CLAEYS, C.; Temperature Influence on Nanowire Tunnel Field Effect Transistors In: 27th Symposium on Integrated Circuits and Systems Design, SBMicro 2012, Brasilia, DF, Brasil, 2012, v. 49, p223-230, 2012.

Artigo de revista em processo de submissão

NEVES, F. S.; AGOPIAN, P. G. D.; MARTINO, J. A.; CRETU, B.; ROOYACKERS, R.; VANDOOREN, A.; SIMOEN, E.; THEAN, A.; CLAEYS, C. Modeling Low Frequency Noise in Vertical GAA Tunnel FETs with Ge Source, IEEE Transactions on Electron Devices.

\section{Premiações}

Best Paper Award - 27th Symposium on Microelectronics Technology and Devices, SBMicro 2012, Brasilia, DF, Brasil, 2012.

Title of work: Temperature Influence on Nanowire Tunnel Field Effect Transistors. 


\section{REFERÊNCIAS}

(1) MOORE, G. E. Cramming more components onto integrated circuits. Electronics Magazine, n. 4, p.114, 1965.

(2) COLINGE, J. P. Silicon-On-Insulator Technology: materials to VLSI, $3^{\text {rd }}$ ed., Massachusetts. Kluwer Academic Publishers, 2004.

(3) COLINGE, J. P. Thin-Film SOI Technology: the solution to many submicron CMOS problems. Technical Digest of EDM, p. 817-820, 1989.

(4) COLINGE, J. P. Thin-film SOI devices: a perspective, Microelectronic Engineering, v. 8, n. 3-4, p. 127-147, 1988.

(5) NAVARRO, C.; BAWEDIN, M.; ANDRIEU, F.; CRISTOLOVEANU, S. Overestimation of Short-Channel Effects Due to Intergate Coupling in Advanced FDSOI MOSFETs. In: IEEE Transactions on Electron Devices, v. 61, n. 9, p. 32743281, 2014.

(6) NAVARRO, C.; BAWEDIN, M.; ANDRIEU, F.; SAGNES, B.; CRISTOLOVEANU, S. Why are SCE overestimated in FD-SOI MOSFETs? In: Solid-State Device Research Conference (ESSDERC), 2010, Proceedings..., p. 304-307, 2013.

(7) CLAEYS, C.; LEONELLI, D.; ROOYACKERS, R.; VANDOOREN, A.; VERHULST, A.; HEYNS, M.; GROESENEKEN, G.; DE GENDT, S. Fabrication and characterization of $\mathrm{Si}$ and hetero-junction tunnel field effect transistors. In: G-COE PICE International Symposium, Tóquio, 2009.

(8) REDDICK W. M.; AMARATUNGA G. A. J. Silicon surface tunnel transistor. Applied. Physics. Letter, v. 67, n.4, p. 494-496, 1995.

(9) NIRSCHL T. et al. The tunneling field effect transistor (TFET) as an add-on for ultralow-voltage analog and digital processes. Electron Devices Meeting, 2004., p. 195198, 2004.

(10) WANG P. F. et al. Complementary tunneling transistor for low power application. Solid State Electron, v. 48, n. 12, p. 2281-2286, 2004. 
(11) APPENZELLER, J.; LIN, Y.; KNOCH, J.; AVOURIS, P.; Band-to-band tunneling in carbon nanotube field-effect transistors, Physical Review Letters, v. 93, n. 19, p. 196805-1-4, 2004.

(12) BHUWALKA, K.; SCHULZE, J.; EISELE, I.; Performance enhancement of vertical tunnel field-effect transistor with SiGe in the $p+$ layer. In: Japanese Journal of Applied Physics, v. 43, n. 7A, p. 4073-4078, 2004.

(13) CHOI W.Y. et al. Tunneling field-effect transistors (TFETs) with subthreshold swing (SS) less than $60 \mathrm{mV} / \mathrm{dec}$. IEEE Electron Devive Letter 2007; v. 28, n. 8, p. 743-745, 2007.

(14) BOUCART, K. ; IONESCU A. M. Length scaling of the double gate tunnel FET with a high-K gate dielectric. Solid State Electron, v. 51, n. 11-12, p. 1500-1507, 2007.

(15) KNOCH, J.; MANTL S.; APPENZELLER, J. Impact of the dimensionality on the performance of tunneling FETs: Bulk versus onedimensional devices. Solid State Electron, v. 51, n. 4, p. 572-578, 2007.

(16) SEABAUGH, A.; ZHANG, Q. Low-voltage tunnel transistors for beyond CMOS logic. Proceedings of the IEEE, v. 28, n. 12, p. 2095-2110, 2010.

(17) FISCHER W. Field Induced Tunnel Diode, IBM Technical Disclosure Bulletin, 1973.

(18) VERHULST, A. S.; VANDENBERGHE W. G.; MAEX, K.; GROESENEKEN, G. Tunnel field-effect transistor without gate-drain overlap. Applied Physics Letters, vol. 91, n. 5, 2007.

(19) CLAEYS, C. et al. Fabrication and Characterization of Si and Hetero-junction Tunnel Field Effect Transistors. In: G-COE PICE International Symposium, Tokyo, 2009.

(20) VERHULST, A. et al. Boosting the on-current of Si-based tunnel field-effect transistors. Abstract in: 218th ECS Meeting. 2010, Las Vegas, NV, USA, v. 2, p.1893, 2010. 
(21) ANGHEL C.; CHILAGANI, P.; AMARA, A.; VLADIMIRESCU, A. Tunnel field effect transistor with increased ON current, low-k spacer and high-k dielectric. Applied Physics Letters, v. 96, n 12, 2010.

(22) VANDOOREN, A. et al. Impact of process and geometrical parameters on the electrical characteristics of vertical nanowire silicon n-TFETs. Solid State Electron, v. 72, p. 82-87, 2012.

(23) ROOYACKERS, R., VANDOOREN, A., VERHULST, A. S., WALKE A., DEVRIENDT K., LOCOROTONDO S., DEMAND M., BRYCE G., LOO R., HIKAVYY A., VANDEWEYER T., HUYGHEBAERT C., COLLAERT N. e THEAN, N. A new complementary hetero-junction vertical TunnelFET integration scheme. IEEE International Electron Devices Meeting, p. 4.2.1-4.2.4, Dec. 2013.

(24) ESAKI, L. New Phenomenon in Narrow Germanium p-n Junctions. In: Physical Review, vl. 109, p. 603, 1958.

(25) HOFSTEIN, S. R.; WARFIELD, G. The insulated gate tunnel junction triode. In: IEEE Transactions on Electron Devices, v. 12, p. 66-76, 1965.

(26) QUINN, J. J.; KAWAMOTO, G.; MCCOMBE, B. D. Subband spectroscopy by surface channel tunneling. In: Surface Science, v. 73, p. 190-196, 1978.

(27) BANERJEE, S.; RICHARDSON, W.; COLEMAN, J.; CHATTERJEE, A. A new three-terminal tunnel device. In: IEEE Electron Device Letters, v. 8, p. 347-349, 1987.

(28) TAKEDA, E.; MATSUOKA, H.; IGURA, Y.; ASAI, S.; A band to band tunneling MOS device (B2T-MOSFET). In: IEDM Technical Digest, p. 402-405, 1988.

(29) BABA, T. Proposal for Surface Tunnel Transistors. In: Japanese Journal of Applied Physics, v. 31, p. L455-L457, 1992.

(30) KANE, E. O. Theory of Tunneling. Journal of Applied Physics, v. 32, n. 1, p. 8391, 1961.

(31) STREetMAN, B. G. Solid States Electronic Devices, $4^{\text {th }}$ ed., New Jersey, EUA, Prentice-Hall, 1995. 
(32) COlinge, J. P.; COLINGE, C.A. Physics of Semiconductor Devices. Massachusetts (EUA), Kluwer Academic Publishers, 2002.

(33) HALL, R.N. Electron-hole recombination in Germanium. Physical Review, v. 87, p. $387,1952$.

(34) SHRODER, D. K. Semiconductor Material and Devices Caracterization, New York, John Wiley \& Sons, 2006.

(35) SCHENK, A. Rigorous Theory and Simplified Model of the Band-to-Band Tunneling in Silicon. Solid-State Electronics, v. 36, n. 1, p. 19-34, 1993.

(36) HURKX, G. A. M.; KLAASSEN, D. B. M.; KNUVERS, M. P. G. A New Recombination Model for Device Simulation Including Tunneling. IEEE Transactions on Electron Devices, v. 39, n. 2, p. 331-338, 1992.

(37) DUKE, C. B. Tunneling in solids. In: Solid State Physics Suplement: Academic Press, v. 10, 1969.

(38) SELBERHERR, S. MOS Device Modeling at 77K. IEEE Transactions on Electron Devices, v. 36, n. 8, p. 1464-1474, 1989.

(39) SZE, S. M. Physics of semiconductor devices, $2^{\text {nd }}$ ed. New York, John Wiley \& Sons, 1981

(40) SCHRODER, D. K. Semiconductor material and device characterization, $3^{\text {rd }}$ ed. New York, John Wiley \& Sons, 2006.

(41) ENEMAN, G., WIOT, M., BRUGÈRE, A., SICART, O., CASAIN, I., SONDE, S. Impact of donor concentration, electric field, and temperature effects on the leakage current in germanium $\mathrm{p}+/ \mathrm{n}$ junctions. IEEE Transaction Electron Devices, v. 55, n. 9, p 2287-2294, 2008.

(42) HAARTMAN, M. V. e OSTLING, M. Low-frequency noise in advanced MOS devices, Netherlands: Springer, 2007.

(43) MCWHORTER, A. L. 1/f noise and related surface effects in germanium. MIT Lincoln Laboratory, Tech Report. n.80, p. 1-122, 1955. 
(44) HOOGE, F. N. 1/f noise sources. IEEE Transaction Electron Devices, v. 41, n. 11, p.1926-1935, 1994.

(45) VANDOOREN, A. et al. Electrical results of vertical Si N-Tunnel FETs. Solid-State Device Research Conference (ESSDERC), p. 255-258, 2011.

(46) ENEMAN, G. et al. Impact of Donor Concentration, Electric Field, and Temperature Effects on the Leakage Current in Germanium $p+/ n$ Junctions. IEEE Transactions on Electron Devices, v. 55, n. 9, 2008.

(47) KRISHNAMOHAN, T.; KIM, D.; RAGHUNATHAN, S.; SARASWAT, K. Doublegate strained-Ge heterostructure tunneling FET (TFET) with record high drive currents and $<60 \mathrm{mV} / \mathrm{dec}$ subthreshold slope. IEDM Technical Digest, p. 947, 2008.

(48) LEONELLI, D.; VANDOOREN, A.; ROOYACKERS, R.; GENDT, S.; HEYNS, M. M.; GROESENEKEN, G. Drive current enhancement in p-tunnel FETs by optimization of the process conditions. Solid-State Electronics, v. 65-66, p. 28, 2011.

(49) LEONELLI, D. et al. Optimization of Tunnel FETs: Impact of Gate Oxide Thickness, Implantation and Annealing Conditions. In: Solid State Device Research Conference (ESSDERC), 2010, Proceeding..., p. 170-173, 2010.

(50) SCHULZE, A. et al. Observation of diameter dependent carrier distribution in nanowire-based transistors. IOP PUBLISHING, Nanotechnology, v. 22, n. 18, 2011.

(51) MALLIK, A. et al. Tunnel field-effect transistors foranalog/mixed-signal system-onchip applications. IEEE Trans. Electron Devices, v. 59, n. 4, p. 888-894, Apr. 2012.

(52) GLAS, F. Critical dimensions for the plastic relaxation of strained axial heterostructures in free-standing nanowires. Physics. Review, v. B 74, p. 121-302 2006

(53) BIJESH, R.; MOHATA, D. e DATTA, S. Flicker noise characterization and analytical modeling of homo and hetero-junction III-V tunnel FETs. DRC 2012, v. 37, n. 1990, p. 6-7, 2012.

(54) Silvaco, Inc. Atlas User's Manual: DEVICE SIMULATIONS SOFTWARE. Santa Clara, CA, USA, 2014. 
(55) ROSAYE, J.-Y., YASUDA, Y, SAKAI, A., MIALHE, P., CHARLES, J.-P., WATANABE, Y. Fast and slow-state traps at the MOSFET oxide interface with a temperature dependent $\mathrm{C}-\mathrm{V}$ method. IEEE Journal of Electron Devices, v.1, p. 1-6, 2003.

(56) KLAASSEN, D. B. M. A Unified Mobility Model for Device Simulation - I. Model Equations and Concentration Dependence, Solid-State Electronics, v. 35, n. 7 , p. 953-959, 1992.

(57) LOMBARDI, C. et al. A Physically Based Mobility Model for Numerical Simulation of Nonplanar Devices. IEEE Transactions on Computer-Aided Design, v. 7, n. 11, p. 1164-1171, 1988.

(58) CANALI, C. et al. Electron and Hole Drift Velocity Measurements in Silicon and Their Empirical Relation to Electric Field and Temperature. IEEE Transactions on Electron Devices, v. ED-22, n. 11, p. 1045-1047, 1975. 


\section{ANEXO A}

\section{Simulador numérico de dispositivos}

Com o intuito de melhor entender o funcionamento dos dispositivos foram utilizadas ferramentas como Technology CAD (Computer-Aided Design), ou simplesmente TCAD.

As ferramentas TCAD englobam um amplo pacote de programas que permitem simular processos de fabricação de um dispositivo, simular a operação de dispositivos bidimensionais ou tridimensionais, visualizar estruturas geradas, curvas, parâmetros físicos e elétricos dos dispositivos e também calibrar os simuladores para melhor aproximação com a realidade.

Neste trabalho foram utilizados o TCAD Sentaurus, desenvolvido pela Synopsys ${ }^{\circledR}$ e o TCAD Silvaco, desenvolvido pela Silvaco ${ }^{\circledR}$.

\section{Modelos utilizados}

A tabela seguinte apresenta o conjunto de modelos adotado com o objetivo aproximar o funcionamento do dispositivo simulado com um dispositivo real, seguido da descrição de cada um deles.

Tabela A-1 - Modelos utilizados no TCAD Sentaurus e TCAD Silvaco

\begin{tabular}{|l|l|}
\hline Sentaurus & Silvaco \\
\hline Phumob & Kla \\
\hline Enormal & CVT \\
\hline HighFieldSaturation & FLDMOB \\
\hline SRH & SRH \\
\hline Avalanche & Impact Selb \\
\hline BandGapNarrowing & BGN \\
\hline Band2Band & BBT.Kane \\
\hline SRH (hurkx) & Trap.tunnel \\
\hline
\end{tabular}

Fonte: Autor 
PhuMob (Philips Unified Mobility Model) / Kla: Modelo de mobilidade unificado Philips, proposto por Klaasen (56). Descreve de forma unificada a mobilidade dos portadores minoritários e majoritários. Também descreve a dependência da mobilidade com a temperatura considerando os mecanismos de degradação da mobilidade devido ao espalhamento por impurezas e portador-portador.

Enormal / CVT: Modelo de degradação da mobilidade nas interfaces proposto por Lombardi (57). Este modelo considera o efeito do alto campo elétrico transversal, que aumenta a interação dos portadores com a interface semicondutor-isolante, aumentando a degradação devido aos mecanismos de espalhamento por fônons acústicos e pela rugosidade na superfície. Além disso, inclui dependências com a temperatura e com a concentração de dopantes.

HighFieldSaturation / FLDMOB: Modelo de mobilidade proposto por Canali (58) que considera o alto campo elétrico, onde a mobilidade não é mais proporcional ao campo elétrico devido a velocidade de saturação dos portadores. Este modelo também considera a dependência com a temperatura.

BandGapNarrowing / BGN: Este é um modelo dependente da temperatura que considera o estreitamento da banda proibida devido ao alto campo elétrico.

SRH (Shockley-Read-Hall): Modelo de geração-recombinação de portadores, este processo envolve a troca de portadores entre a banda de condução e de valência descrito pela equação 2.1 (33). Foi utilizado com dois sub modelos, DopingDep e TempDep, que consideram, respectivamente os efeitos da concentração de dopantes e o efeito da temperatura em conjunto com tempo de vida dos portadores na recombinação.

SRH (Hurkx) / Trap.tunnel: O tunelamento induzido por armadilhas é implementado matematicamente por uma correção do modelo de recombinação e geração $\mathrm{SRH}$, conforme apresentado no capítulo 2.3.2.1, dado pela equação 2.2.

Band2Band / BBT.Kane: Modelo que considera o tunelamento direto de banda para banda, conforme descrito no capítulo 2.3.2.2, dado pela equação 2.7. 


\section{ANEXO B}

\section{Exemplo de um script utilizado para gerar uma estrutura em TCAD Sentaurus}

(sde:clear)

;;:DEFINIÇỐES

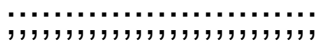

;NOME

(define nome "TFET")

;DOPAGEM

(define NFonte $5 e+19$ )

(define NCanal $5 e+15$ )

(define NDreno 1e+20)

(define DopFonte "BoronActiveConcentration")

(define DopDreno "ArsenicActiveConcentration")

(define DopCanal "ArsenicActiveConcentration")

;MATERIAL

(define MatFonte "SiliconGermanium")

(define MatCanal "Silicon")

(define MatDreno "Silicon")

(define MatOxido "SiO2")

\section{, ,,,,,,,,,,,,,,,,,,,,,,,,,,,,,,}

;;;DIMENSÕES

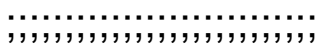

;Comprimento de canal

(define D 0.2)

;Comprimento de canal

(define Ich 0.2)

;Extens $\tilde{A} \mu e s$ de fonte e dreno (define Ifd 0.1)

;Expessura do $\tilde{A}^{3} x i d o$ de porta (define tox 0.0015)

;Overlap/Autoalinhado Fonte (define loxf 0.025) 
;Under/Autoalinhado Dreno

(define loxd -0.1)

;Angulo de corte da fatia

(define $\mathrm{ft}$ 10)

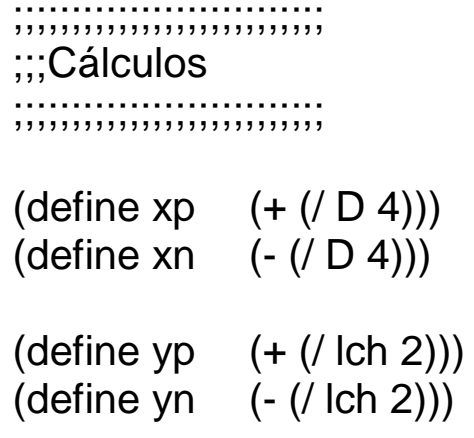




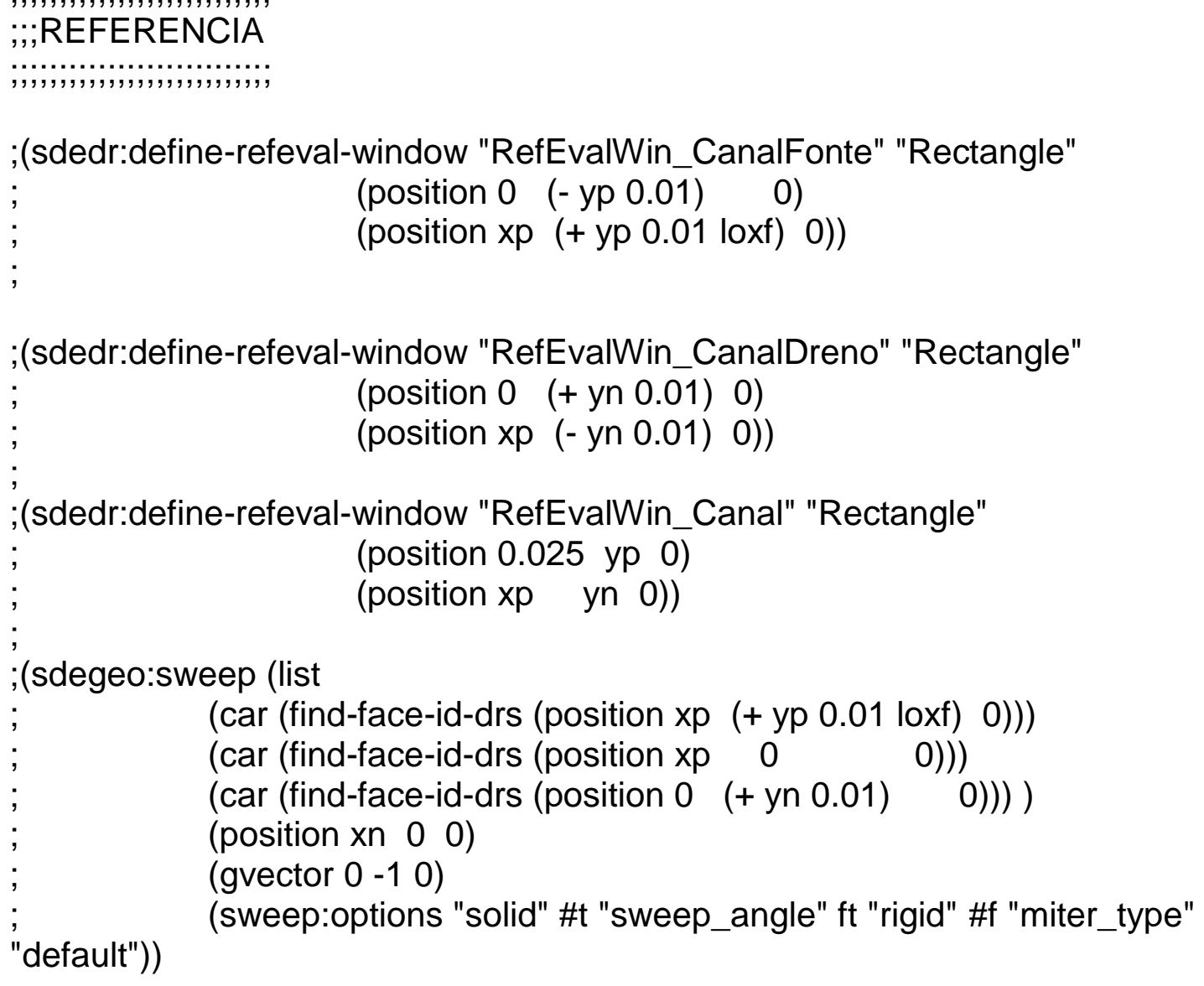




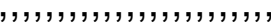

;;:DOPAGEM

;,;,;,;,;,;,,;,;,;,;,;,;,;,;

(sdedr:define-constant-profile "ConstantProfileDefinition_Fonte" DopFonte NFonte) (sdedr:define-constant-profile-region "ConstantProfilePlacement_Fonte"

"ConstantProfileDefinition_Fonte" "fonte")

(sdedr:define-constant-profile "ConstantProfileDefinition_Canal" DopCanal NCanal) (sdedr:define-constant-profile-region "ConstantProfilePlacement_Canal1"

"ConstantProfileDefinition_Canal" "canal1")

(sdedr:define-constant-profile "ConstantProfileDefinition_Canal" DopCanal NCanal) (sdedr:define-constant-profile-region "ConstantProfilePlacement_Canal2"

"ConstantProfileDefinition_Canal" "canal2")

(sdedr:define-constant-profile "ConstantProfileDefinition_Dreno" DopDreno NDreno) (sdedr:define-constant-profile-region "ConstantProfilePlacement_Dreno"

"ConstantProfileDefinition_Dreno" "dreno")

,,,,,,,,,,,,,,,,,,,,,,,,,;,;

;;:;CONTATOS

;,;,;,;,;,;,;,;,;,;,;,;,;,;

(sdegeo:define-contact-set "contato_fonte" 4 (color:rgb 100 ) "\#\#" )

(sdegeo:define-contact-set "contato_dreno" 4 (color:rgb 100 ) "\#\#" )

(sdegeo:define-contact-set "contato_porta" 4 (color:rgb 100 ) "\#\#" )

(sdegeo:set-current-contact-set "contato_fonte")

(sdegeo:define-3d-contact (list (car (find-face-id (position 0 (+ yp Ifd) 0.004)))) "contato_fonte")

(sdegeo:set-current-contact-set "contato_dreno")

(sdegeo:define-3d-contact (list (car (find-face-id (position 0 (- yn lfd) 0.004))))

"contato_dreno")

(sdegeo:set-current-contact-set "contato_porta")

(sdegeo:define-3d-contact (list (car (find-face-id (position (+ xp tox) (+ yp loxf)

0.0001)))) "contato_porta") 
;INTERFACE

;,;,;,;,;,;,;,;,;,;,;;

(sdenoffset:create-noffset-interface "region" "canal1" "fonte" "hlocal" 0.001 "factor" 1.0)

(sdenoffset:create-noffset-interface "region" "fonte" "canal1" "hlocal" 0.001 "factor" 1.3)

(sdenoffset:create-noffset-interface "region" "canal2" "dreno" "hlocal" 0.001 "factor" 1.0)

(sdenoffset:create-noffset-interface "region" "dreno" "canal2" "hlocal" 0.001 "factor" 1.3)

(sdenoffset:create-noffset-interface "region" "canal1" "canal2" "hlocal" 0.0004 "factor" 1.4)

(sdenoffset:create-noffset-interface "region" "canal2" "canal1" "hlocal" 0.0004 "factor" 1.4)

(sdenoffset:create-noffset-interface "region" "OxidoContato" "canal1" "hlocal" 0.0002 "factor" 1.01)

(sdenoffset:create-noffset-interface "region" "canal1" "OxidoContato" "hlocal" 0.001 "factor" 1.15)

(sdenoffset:create-noffset-interface "region" "oxido_lump_1" "canal2" "hlocal" 0.0002 "factor" 1.01)

(sdenoffset:create-noffset-interface "region" "canal2" "oxido_lump_1" "hlocal" 0.001 "factor" 1.15)

(sdenoffset:create-noffset-block "region" "canal1" "maxedgelength" 0.005 "maxlevel" 50)

(sdenoffset:create-noffset-block "region" "canal2" "maxedgelength" 0.005 "maxlevel" 50)

(sdedelaunizer:set-parameters "type" "conforming" "maxPoints" 40000) 


\section{Exemplo de um script de simulação em TCAD Sentaurus}

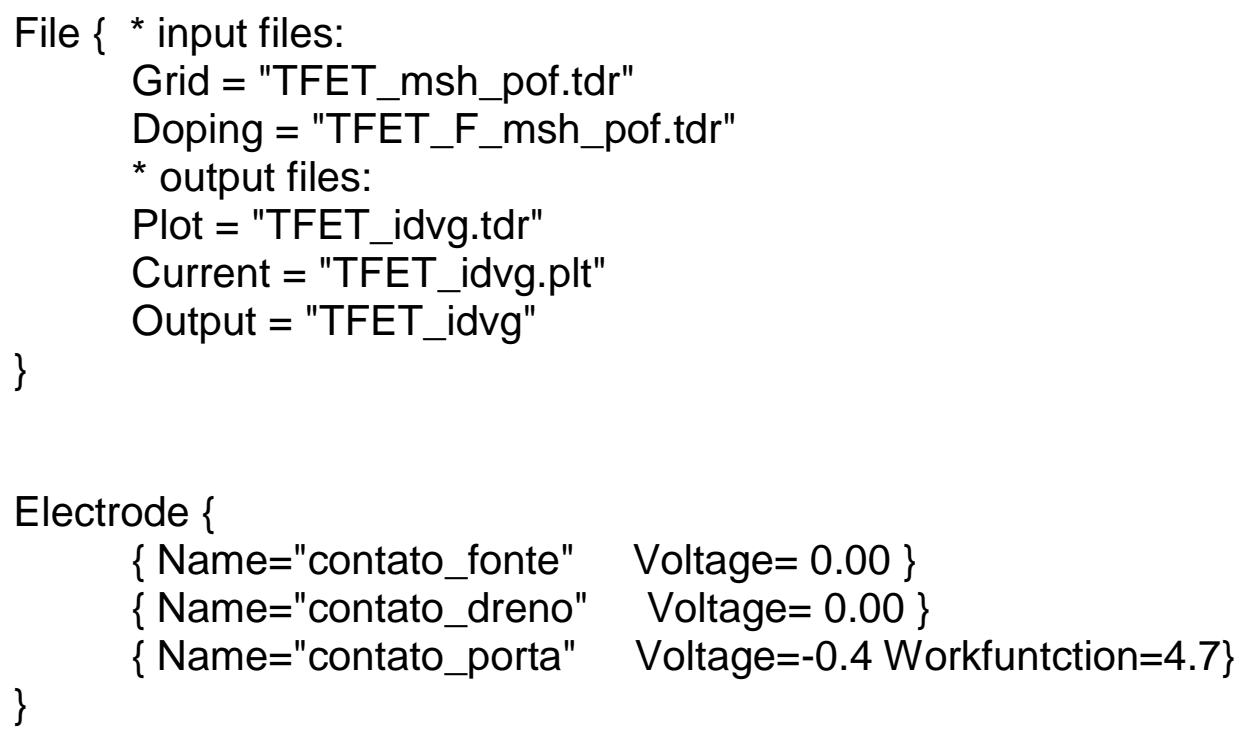


Plot\{

eDensity hDensity

TotalCurrent/Vector eCurrent/Vector hCurrent/Vector

eMobility hMobility

eVelocity hVelocity

eQuasiFermi hQuasiFermi

eTemperature Temperature hTemperature

ElectricField/Vector Potential SpaceCharge

Doping DonorConcentration AcceptorConcentration

SRH Band2Band Auger

AvalancheGeneration eAvalancheGeneration hAvalancheGeneration

eGradQuasiFermi/Vector hGradQuasiFermi/Vector

eEparallel hEparallel eENormal hENormal

BandGap

BandGapNarrowing

Affinity

ConductionBand ValenceBand

eBarrierTunneling hBarrierTunneling * BarrierTunneling

eTrappedCharge hTrappedCharge

eGapStatesRecombination hGapStatesRecombination

eDirectTunnel hDirectTunnel

\}

Math \{

Extrapolate

RelErrControl

Iterations $=50$

Method $=$ pardiso

Number_Of_Threads $=$ maximum

StackSize $=200000000$

\}

wallclock

Solve \{

\#-initial solution:

Poisson

Coupled \{ Poisson Electron hole \}

\#-ramp gate:

Quasistationary ( InitialStep $=0.02$ Maxstep $=0.02$ Minstep $=1$ e-5

Goal $\{$ Name="contato_dreno" Voltage $=0.9\}$ )

\{ Coupled $\{$ Poisson Electron hole\} $\}$

Quasistationary ( InitialStep $=0.004167$ Maxstep $=0.004167$ Minstep $=1$ e-5

Goal $\{$ Name="contato_porta" Voltage $=2.0\}$ )

\{ Coupled $\{$ Poisson Electron hole\} $\}$ 
Exemplo de um script de simulação em TCAD Silvaco

GO ATLAS

\#

MESH SPACE.MULT $=1.0$

$\#$

X.MESH LOC $=-0.215$ SPAC $=0.005$

X.MESH LOC $=-0.210$ SPAC $=0.005$

X.MESH LOC $=-0.110$ SPAC $=0.001$

X.MESH LOC $=-0.05$ SPAC $=0.001$

X.MESH LOC $=-0.025 \quad S P A C=0.005$

X.MESH LOC $=0 \quad S P A C=0.005$

X.MESH LOC $=0.025$ SPAC $=0.0005$

X.MESH LOC $=0.055$ SPAC $=0.001$

X.MESH LOC $=0.075$ SPAC $=0.001$

X.MESH LOC $=0.09$ SPAC $=0.001$

X.MESH LOC $=0.210$ SPAC $=0.005$

X.MESH LOC $=0.215$ SPAC $=0.005$

Y.MESH LOC $=-0.103$ SPAC $=0.002$

Y.MESH LOC $=-0.100$ SPAC $=0.001$

Y.MESH LOC $=-0.095$ SPAC $=0.002$

Y.MESH LOC $=-0.085 \quad S P A C=0.002$

Y.MESH LOC $=-0.07 \quad S P A C=0.005$

Y.MESH LOC $=0 \quad S P A C=0.005$

Y.MESH LOC $=0.07 \quad$ SPAC $=0.005$

Y.MESH LOC $=0.085 \quad S P A C=0.002$

Y.MESH LOC $=0.095$ SPAC $=0.002$

Y.MESH LOC $=0.100$ SPAC $=0.001$

Y.MESH LOC $=0.103$ SPAC $=0.002$ 


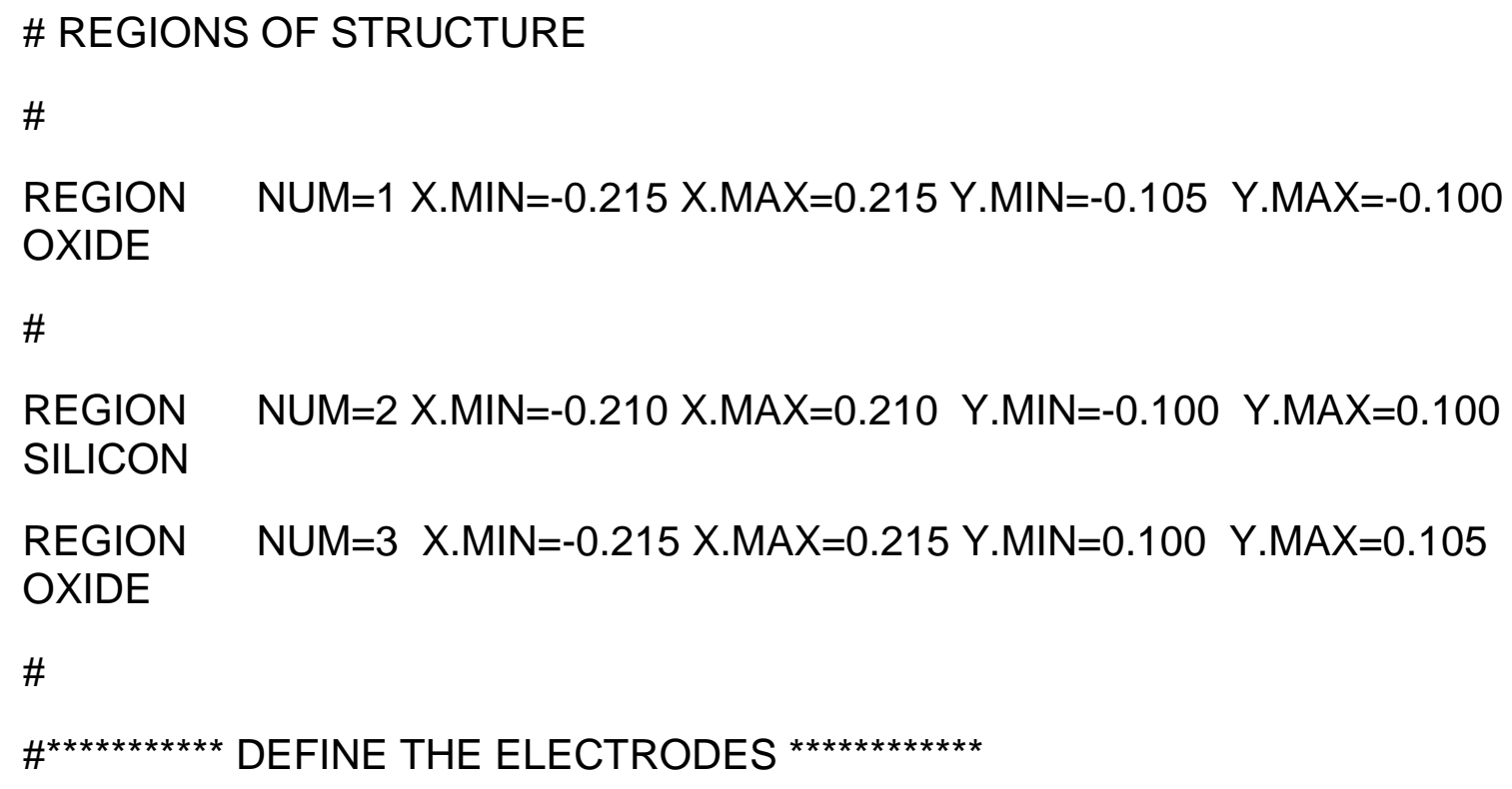


\#STRUCTURE OUTF=TFET.STR

\# SET WORKFUNCTION OF GATE

CONTACT NAME $=$ GATE N.POLY WORKFUNC $=4.7$

CONTACT NAME=GATE2 N.POLY COMMON=GATE WORKFUNC $=4.7$

\#

\# SELECT MODELS

MODELS KLA SHI BGN KLAAUG FLDMOB FERMI DRIFT.DIFF PRINT BBT.KANE SRH TEMP=300 TRAP.TUNNEL

\#

SOLVE INIT

\#

\# DO IDVG CHARACTERISTIC

\#

METHOD NEWTON AUTONR TRAP MAXTRAP $=10$

SOLVE PREV

SOLVE VDRAIN $=0.05$

SOLVE VDRAIN $=0.1$

SOLVE VDRAIN $=0.4$

SOLVE VDRAIN $=0.6$

SOLVE VDRAIN $=0.9$

\#

\# RAMP GATE VOLTAGE

\#

LOG OUTF=TFET.LOG

SOLVE VGATE $=0$ VSTEP $=0.05$ NAME=GATE VFINAL=2 